\title{
Non-abelian unipotent periods Monodromy of iterated integrals
}

\author{
Zdzisław Wojtkowiak
}

\section{$\S 0$. Introduction.}

0.1. Let $X$ be a smooth, algebraic variety defined over a number field $k$. Let $\sigma: k \hookrightarrow \mathbb{C}$ be an inclusion. We set $X_{\mathbb{C}}=X \underset{k}{\times \mathbb{C}}$. Let $X(\mathbb{C})$ be the set of $\mathbb{C}$-points of $X_{\mathbb{C}}$ with its complex topology. There is the canonical isomorphism

$$
p_{\text {comp }}: H_{B}^{n}(X(\mathbb{C})) \otimes \mathbb{C} \stackrel{\approx}{\longrightarrow} H_{D R}^{n}(X) \underset{k}{\otimes} \mathbb{C}
$$

between Betti (singular) cohomology and algebraic De Rham cohomology. The period $\operatorname{matrix}\left(p_{i j}\right)$ is defined by equations $\omega_{i}=\sum p_{j i} \sigma_{j}$ where $\left\{\omega_{i}\right\}$ and $\left\{\sigma_{i}\right\}$ are bases of $H_{D R}^{n}(X)$ and $H_{B}^{n}(X(\mathbb{C}))$ or $p_{j i}=\int_{\sigma_{j}^{*}} \omega_{i}$ where $\left\{\sigma_{j}^{*}\right\}$ is the dual base of $H_{n}(X(\mathbb{C}))$.

Let us assume that $X$ is an abelian variety. Let $G$ be the largest subgroup of $G L\left(H_{B}^{1}(X(\mathbb{C})) \otimes \mathbb{Q}\right) \times G_{m}$ which fixes all tensors $c l_{B}(Z)$, where $Z$ is an algebraic cycle on some $X^{n}$ (see [D2]). Let $P$ be the functor of $k$-algebras, such that any element of $P(A)$ is an isomorphism $p: H_{B}^{1}(X(\mathbb{C})) \otimes A \rightarrow H_{D R}^{1}(X) \otimes A$ mapping $c l_{B}(Z) \otimes 1$ to $c l_{D R}(Z) \otimes 1$ for any algebraic cycle $Z$ on any $X^{n}$. The isomorphism $p_{\text {comp }}$ belongs to $P(\mathbb{C})$, the functor $P$ is represented by an algebraic variety over $k$, which is a $G_{k}$-torsor under the natural action. It is a subtorsor of the $G L\left(H_{B}^{1}(X(\mathbb{C}) \otimes k)\right.$-torsor $\operatorname{Iso}\left(H_{B}^{1}(X(\mathbb{C})) \otimes k, H_{D R}^{1}(X)\right)$.

Let $T$ be a smallest subtorsor defined over $k$ of the torsor $\operatorname{Iso}\left(H_{B}^{1}(X(\mathbb{C})) \otimes k, H_{D R}^{1}(X)\right)$, which contains $p_{\text {comp }}$ as a $\mathbb{C}$-point and let $\mathcal{G}$ be the corresponding subgroup (defined over $k)$ of $G L\left(H_{B}^{1}(X(\mathbb{C})) \otimes k\right)$. Let $Z\left(p_{\text {comp }}\right)$ be the Zariski closure of $p_{\text {comp }}$ in $\operatorname{Iso}\left(H_{B}^{1}(X(\mathbb{C}) \otimes\right.$ $\left.k, H_{D R}^{1}(X)\right)$ i.e. the smallest Zariski closed subset defined over $k$, which contains $p_{\text {comp }}$ as a $\mathbb{C}$-point.

Then we have

$$
Z\left(p_{\text {comp }}\right) \subset T \subset P
$$


and

$$
\mathcal{G} \subset G
$$

In order to calculate $Z\left(p_{\text {comp }}\right)$ and to show that $Z\left(p_{\text {comp }}\right)=P$ one need to show that certain number are transcendental. On the other hand to calculate $T$ and $\mathcal{G}$ seems to be an easier task. The requirement that $T$ is a subtorsor of $\operatorname{Iso}\left(H_{B}^{1}(X(\mathbb{C})) \otimes k, H_{D R}^{1}(X)\right)$ is very strong and usually relatively weak informations about numbers $\left\{p_{i j}\right\}$ are necessary to calculate $T$ and $\mathcal{G}$. We give an obvious example. If $X=P_{\mathbb{Q}}^{1}$ then in order to show that $Z\left(p_{\text {comp }}\right) \approx G_{m}$ we must know that $2 \pi i$ is transcendental. But already the fact that $2 \pi i$ is not a $k^{\text {th }}$-root of a rational number for any $k \in N$ implies that $T \approx G_{m}$ and $\mathcal{G}=G_{m}$.

In this note we shall discuss periods for fundamental groups. We shall concentrate on analogues of $T$ and $\mathcal{G}$ for fundamental groups. On the other hand we have no analogue of $P$ and $G$.

The plan of the paper.

0. Introduction.

1. Torsors.

2. Torsors associated to non-abelian unipotent periods.

3. Canonical connection with logarithmic singularities.

4. The Gauss-Manin connection associated with the morphism $X^{\Delta[1]} \rightarrow X^{\partial \Delta[1]}$ of cosimplicial schemes.

5. Torsors associated to the canonical unipotent connection with logarithmic singularities.

6. Partial informations about $\mathcal{G}_{D R}\left(P^{1}(\mathbb{C}) \backslash\{0,1, \infty\}\right)$.

7. Homotopy relative tangential base points on $P^{1}(\mathbb{C}) \backslash\left\{a_{1}, \ldots, a_{n+1}\right\}$.

8. Generators of $\pi_{1}\left(P^{1}(\mathbb{C}) \backslash\left\{a_{1}, \ldots, a_{n+1}\right\}, x\right)$.

9. Monodromy of iterated integrals on $P^{1}(\mathbb{C}) \backslash\left\{a_{1}, \ldots, a_{n+1}\right\}$.

10. Calculations.

11. Configuration spaces.

12. The Drinfeld-Ihara $Z / 5$-cycle relation. 
13. Functional equations of iterated integrals.

14. Subgroups of $\operatorname{Aut}\left(\pi^{2}\right)$.

A.1. Malcev completion.

The dependence of sections

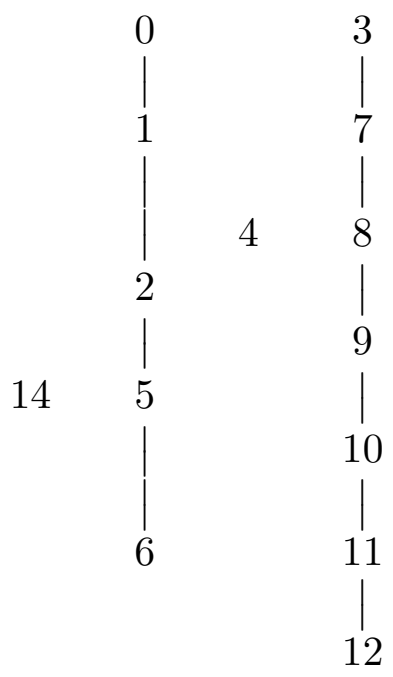

0.2. Below we shall briefly discuss the contents of the paper. Let us assume that $X$ is a smooth, quasi-projective, algebraic variety defined over a number field $k$. Let $x$ be a $k$ point of $X$. In [W1] we defined affine, connected, pro-unipotent group schemes over $k$ and $\mathbb{Q}$ respectively; $\pi_{1}^{\mathrm{DR}}(X, x)$ - the algebraic De Rham fundamental group and $\pi_{1}^{B}(X(\mathbb{C}), x)$ — the Betti fundamental group. We have also the inclusion (of $\mathbb{Q}$-points into $\mathbb{C}$-points)

$$
\Phi_{x}: \pi_{1}^{B}(X(\mathbb{C}), x)(\mathbb{Q}) \rightarrow \pi_{1}^{\mathrm{DR}}(X, x)(\mathbb{C})
$$

such that the induced homomorphism on $\mathbb{C}$-points

$$
\varphi_{x}: \pi_{1}^{B}(X(\mathbb{C}), x)(\mathbb{C}) \rightarrow \pi_{1}^{\mathrm{DR}}(X, x)(\mathbb{C})
$$

is an isomorphism.

The affine, pro-algebraic scheme over $k$

$$
\text { Iso }:=\operatorname{Iso}\left(\pi_{1}^{B}(X(\mathbb{C}), x) \times k, \pi_{1}^{\mathrm{DR}}(X, x)\right)
$$


is an $\operatorname{Aut}\left(\pi_{1}^{\mathrm{DR}}(X(\mathbb{C}), x)\right)$-torsor. Let $Z\left(\varphi_{x}\right)$ be the Zariski closure of $\varphi_{x}$ in Iso. Let $T\left(\varphi_{x}\right)$ be the smallest subtorsor of Iso, defined over $k$ which contains $\varphi_{x}$ as a $\mathbb{C}$-point. Let $G_{\mathrm{DR}}\left(\varphi_{x}\right) \subset \operatorname{Aut}\left(\pi_{1}^{\mathrm{DR}}(X, x)\right)$ be the corresponding subgroup. One can hope that

$$
Z\left(\varphi_{x}\right)=T\left(\varphi_{x}\right)
$$

We shall denote by $\mathcal{G}_{\mathrm{DR}}(X)$ the image of $G_{\mathrm{DR}}\left(\varphi_{x}\right)$ in $\operatorname{Out}\left(\pi_{1}^{\mathrm{DR}}(X, x)\right)$.

The calculation of the homomorphism $\varphi_{x}$ is equivalent to the calculation of the monodromy of all iterated integrals on $X$. We shall see that the monodromy representation of iterated integrals have a lot of properties similar to the action of $\operatorname{Gal}(\bar{k} / k)$ on fundamental groups. For example if $S$ is a loop on a curve around a missing point, then " $\sigma(S) \sim S^{\chi(\sigma) "}$ in the $l$-adic case and " $\theta(S) \sim S^{-2 \pi i}$ " for iterated integrals. Let $\varphi: G_{k}:=\operatorname{Gal}(\bar{k} / k) \rightarrow \operatorname{Out}\left(\pi_{1}(\underset{k}{\times} \bar{k}, x)_{(l)}\right)$ be the natural homomorphism. Very optimistically we can state the following conjecture:

$$
\operatorname{Lie}\left(\varphi\left(G_{k}\right)\right) \otimes k \approx \operatorname{Lie}\left(\mathcal{G}_{\mathrm{DR}}(X)\right) \otimes \mathbb{Q}_{l}
$$

We also point out that (0.1) and (0.2) will imply that values of the Riemann zeta function at odd integers are all transcendental over $\mathbb{Q}(-2 \pi i)$.

0.3. The calculations of non-abelian unipotent periods are in fact the calculations of monodromy of iterated integrals. This causes that the paper contains in fact two different papers. In one part (sections $3,7,8,9,10,11,12,13$ ) we are studying monodromy of iterated integrals. In Section 3 are established some general properties of the canonical unipotent connection. In Section 7 we present a "naive" approach to the Deligne tangential base point, which is sufficient for our applications. In Sections 9 and 10 we describe the monodromy of iterated integrals on $P^{1}(\mathbb{C}) \backslash\left\{a_{1}, \ldots, a_{n+1}\right\}$ and in more details on $P^{1}(\mathbb{C}) \backslash\{0,1, \infty\}$. In Sections $11-13$ we study monodromy of iterated integrals on configuration spaces. We give a proof of Drinfeld-Ihara $Z / 5$-cycle relation which is different from the proof in [Dr]. The proof should be (is) analogous to the proof in [I2]. We point out that the main point in our proof is the functoriality property of the universal unipotent 
connection. This property can be shortly written as $f_{*} \omega=f^{*} \omega$ and it is also fundamental in our results on functional equations of polylogarithms and iterated integrals (see [W4]).

In "the second paper" contained in this paper we discuss torsors and corresponding groups associated to non-abelian unipotent periods. In Section 1 we give some general results and definitions. In Section 2 we define a torsor and a corresponding group associated to non-abelian unipotent periods and we state some conjectures related to Galois representations on fundamental groups. In Section 5 we define a torsor associated to the monodromy of iterated integrals. Using results from Section 4 we show that this torsor and the corresponding group coincides with the ones from Section 2. In Section 6 we calculate some part of the group $\mathcal{G}_{\mathrm{DR}}(\mathbb{X})$ for $\mathbb{X}=\mathbb{P}^{1}(\mathbb{C}) \backslash\{0,1, \infty\}$. This part corresponds to the Galois representation on $\pi_{1}(\mathbb{X}, \overrightarrow{01}) /\left[\pi^{\prime}, \pi^{\prime}\right]$ where $\pi^{\prime}:=\left[\pi_{1}(\mathbb{X}, \overrightarrow{01}), \pi_{1}(\mathbb{X}, \overrightarrow{01})\right]$ (see [I3]). About this part of $\mathcal{G}_{\mathrm{DR}}(\mathbb{X})$, let us call it $G$, we have the following result.

The group $G$ contains a group $H=\left\{f_{t}\left|f_{t}(X)=t \cdot X, f_{t}(Y)=t \cdot Y\right| t \in \mathbb{C}^{*}\right\}$ if and only if all numbers $\zeta(2 k+1)$ are irrational. This leads to a definition of a new group associated to unipotent periods, which should be relatively easily calculated. This new group is not considered in this paper, see however Corollary 6.4 and Theorem 6.7 iii).

Acknowledgment. We would like to thank Professor Deligne, who once showed the one-form considered in Section 3 in the case of $\mathbb{C} \backslash\{0,1\}$. Thanks are due to Professor Y. Ihara for showing us his proof of 5-cycle relation, which help us to find an analogous one for unipotent periods. We would like to express our thanks to Professor Hubbuck for his invitation to Aberdeen, where Section 12 was written and where in May 1993 we had a possibility to give seminar talks on 5-cycle relation for unipotent periods.

We would like to thank very much Professor Y. Ihara for his invitation to Kyoto. We would like to thank Professors Oda, Matsumoto, Tamagawa for useful discussions and comments during my seminar talks.

Finally thanks are due to Professor L. Lewin, who once invited us to write a chapter in the book on polylogarithms and suggested to include also results about monodromy of iterated integrals. This encourage us very much to continue to work on this subject (see preprints [W2] and [W3] which some parts are included in the present paper). 


\section{$\S 1$. Torsors.}

Let $G_{1}$ and $G_{2}$ be two groups. We say that a set $T$ is a $\left(G_{1}, G_{2}\right)$-bitorsor if $T$ is equipped with a free, transitive, left action of $G_{1}$ and with a free, transitive right action of $G_{2}$ and if the actions of $G_{1}$ and $G_{2}$ commute.

We say that a subset $S \subset T$ is a subtorsor of $T$ if there exist subgroups $H_{1} \subset G_{1}$ and $H_{2} \subset G_{2}$ such that $S$ is a $\left(H_{1}, H_{2}\right)$-bitorsor under the natural actions of $H_{1}$ and $H_{2}$.

We say that a subset $S \subset T$ is a left (resp. right) subtorsor of $T$ if there is a subgroup $H_{1} \subset G_{1}$ (resp. $H_{2} \subset G_{2}$ ) such that the natural action of $H_{1}$ (resp. $H_{2}$ ) on $S$ is free and transitive.

Main example. Let $G_{1}$ and $G_{2}$ be two groups. Assume that $G_{1}$ and $G_{2}$ are isomorphic. Then the set of isomorphisms from $G_{1}$ to $G_{2}$, which we denote by $\operatorname{Iso}\left(G_{1}, G_{2}\right)$, is an $\left(\operatorname{Aut}\left(G_{1}\right), \operatorname{Aut}\left(G_{2}\right)\right)$-bitorsor.

For any non-empty subset $S \subset \operatorname{Iso}\left(G_{1}, G_{2}\right)$, the intersection of all subtorsors (resp. right subtorsors, resp. left subtorsors) of Iso $\left(G_{1}, G_{2}\right)$, which contain $S$, is a subtorsor (resp. right subtorsor, resp. left subtorsor) of Iso $\left(G_{1}, G_{2}\right)$, which we denote by $T(S)$ (resp. $T_{r}(S)$, resp. $\left.T_{l}(S)\right)$.

\subsection{Unipotent, affine, algebraic groups and torsors.}

Let $k$ be a field of characteristic zero. We say that $X$ is an algebraic variety (defined) over $k$ if $X$ is an algebraic scheme over Spec $k$. If $A$ is a $k$-algebra, we set $X_{A}:=$

$X \underset{\text { Spec } k}{\times} \operatorname{Spec} A$. The set of $A$-points of $X$ we denote by $X(A)$. We say that $G$ is an affine, algebraic group (defined) over $k$, if $G$ is an affine, algebraic group scheme over Spec $k$.

1.1.1. Let $G$ be an affine, unipotent, connected, algebraic group over $k$. Then there is an affine, algebraic group $\operatorname{Aut}(G)$ over $k$ such that for any $k$-algebra $A$ we have

$$
\operatorname{Aut}(G)(A)=\operatorname{Aut}\left(G_{A}\right)
$$


Let $G_{1}$ and $G_{2}$ be two connected, affine, unipotent, algebraic groups over $k$. Then there is a smooth, affine, algebraic variety $\operatorname{Iso}\left(G_{1}, G_{2}\right)$ over $k$, such that for any $k$-algebra $A$ we have

$$
\operatorname{Iso}\left(G_{1}, G_{2}\right)(A):=\operatorname{Iso}\left(G_{1 A}, G_{2 A}\right) .
$$

Proof of 1.1.1. Let $\mathcal{G}$ be a Lie algebra of $G$. The exponential map exp: $\mathcal{G} \rightarrow G$ is an isomorphism of affine, algebraic groups if we equip $\mathcal{G}$ with a group law given by the Baker-Campbell-Hausdorff formula. The automorphisms of the group $\mathcal{G}$ coincides with the automorphisms of the Lie algebra $\mathcal{G}$. One can easily give an ideal defining $\operatorname{Aut}_{\text {Lie }}(\mathcal{G})$ in $k[G L(\mathcal{G})]$. In the similar way one constructs $\operatorname{Iso}\left(G_{1}, G_{2}\right)$.

1.1.2. In $[\mathrm{S}]$ page 149 there is a definition of a (left) $G$-torsor (a principal homogeneous space of $G$ ) if $G$ is a linear, algebraic group over $k$. This definition extends immediately for bitorsors of algebraic groups.

We say that an affine, algebraic variety $T$ over $k$ is a $\left(G_{1}, G_{2}\right)$-bitorsor, if there are morphisms $G_{1} \times T \rightarrow T$ and $T \times G_{2} \rightarrow T$ over Spec $k$, which define free, transitive actions of $G_{1}$ and $G_{2}$ on $T$ and if these actions commute.

Observe that if $T$ has a $k$-point then $G_{1}$ and $G_{2}$ are isomorphic.

The definitions of a subtorsor, a right subtorsor and a left subtorsor we left to a reader, as well as a proof of the following lemma.

Lemma 1.1.2. Let $T_{1}$ be an $\left(H_{1}, H_{2}\right)$-subtorsor of $T$ and let $T_{2}$ be an $\left(H_{1}^{\prime}, H_{2}^{\prime}\right)$-subtorsor of $T$. Assume that $T_{1} \cap T_{2} \neq \emptyset$. Then the intersection $T_{1} \cap T_{2}$ is an $\left(H_{1} \cap H_{2}, H_{1}^{\prime} \cap H_{2}^{\prime}\right)$ subtorsor of $T$. The similar statements hold for right and left subtorsors of $T$.

1.1.3. Main example. Let $G_{1}$ and $G_{2}$ be two connected, unipotent, affine, algebraic groups over $k$. Assume that there is an isomorphism $G_{1 \bar{k}} \rightarrow G_{2 \bar{k}}$. Then the algebraic variety $\operatorname{Iso}\left(G_{1}, G_{2}\right)$ is an $\left(\operatorname{Aut}\left(G_{1}\right), \operatorname{Aut}\left(G_{2}\right)\right)$-bitorsor, if we equip $\operatorname{Iso}\left(G_{1}, G_{2}\right)$ with the obvious actions of $\operatorname{Aut}\left(G_{1}\right)$ and $\operatorname{Aut}\left(G_{2}\right)$.

Let $k \subset \mathbb{C}$ be a subfield of $\mathbb{C}$. Let $\Theta: G_{1}(\mathbb{C}) \rightarrow G_{2}(\mathbb{C})$ be an isomorphism. Then $\Theta$ is a $\mathbb{C}$-point of $\operatorname{Iso}\left(G_{1}, G_{2}\right)$. We denote by $Z(\Theta)$ the Zariski closure of $\Theta \operatorname{in} \operatorname{Iso}\left(G_{1}, G_{2}\right)$ 
i.e. the smallest algebraic subset of $\operatorname{Iso}\left(G_{1}, G_{2}\right)$ (defined) over $k$, which contains $\Theta$ as a $\mathbb{C}$-point.

The connected, unipotent, affine, algebraic group $G_{i}$ is isomorphic as an algebraic variety over $k$ to the affine space $A_{k}^{m}$, hence $\Theta$ can be view as a $\mathbb{C}$-point $\left(\Theta_{i j}\right)_{1 \leq i, j \leq n}$ of $A_{k}^{m^{2}}$. Let $k(\Theta)$ be a subfield of $\mathbb{C}$ generated over $k$ by all $\Theta_{i, j}$.

Lemma 1.1.3. The field $k(\Theta)$ does not depend on the choice of isomorphisms $G_{i} \approx A_{k}^{m}$ and the transcendental degree of the field $k(\Theta)$ over $k$ is equal to the dimension of $Z(\Theta)$.

Proof. This follows (is) Lemma 1.7 in [D2].

Definition-Proposition 1.1.4. Let $T(\Theta)$ (resp. $T_{r}(\Theta)$, resp. $T_{l}(\Theta)$ ) be the intersection of all subtorsors $T$ (resp. right-subtorsors $T_{r}$, resp. left subtorsors $T_{l}$ ) defined over $k$ of Iso $\left(G_{1}, G_{2}\right)$, which contain $\Theta$ as a $\mathbb{C}$-point. Then $T(\Theta)$ (resp. $T_{r}(\Theta)$ resp. $T_{l}(\Theta)$ ) is a $\left(G_{r}^{\mathrm{bi}}(\Theta), G_{l}^{\mathrm{bi}}(\Theta)\right)$-subtorsor (resp. right $G_{r}(\Theta)$-subtorsor, resp. left $G_{l}(\Theta)$-subtorsor) of $\operatorname{Iso}\left(G_{1}, G_{2}\right)$ for some $G_{r}^{\mathrm{bi}}(\Theta) \subset \operatorname{Aut}\left(G_{2}\right)$ and $G_{l}^{\mathrm{bi}}(\Theta) \subset \operatorname{Aut}\left(G_{1}\right)\left(\operatorname{resp.} G_{r}(\Theta) \subset \operatorname{Aut}\left(G_{2}\right)\right.$, resp. $\left.G_{l}(\Theta) \subset \operatorname{Aut}\left(G_{1}\right)\right)$.

Proof. The intersection of a family of algebraic varieties coincides with an intersection of a finite number of them. Hence it follows from Lemma 1.1.2 that $T(\Theta), T_{r}(\Theta)$ and $T_{l}(\Theta)$ exist and are unique. The groups are also unique because they are intersections of the corresponding subgroups of $\operatorname{Aut}\left(G_{i}\right)$.

If $T(\Theta)$ has a $k$-point $f$, then $G_{l}^{\mathrm{bi}}(\Theta)=f^{-1} \circ G_{r}^{\mathrm{bi}}(\Theta) \circ f$. If $T_{l}(\Theta)$ (resp. $T_{r}(\theta)$ ) has a $k$ point, then $T(\Theta)=T_{l}(\Theta)$ and $G_{l}^{\text {bi }}(\Theta)=G_{l}(\Theta)\left(\right.$ resp. $T(\Theta)=T_{r}(\Theta)$ and $G_{r}^{\text {bi }}(\Theta)=G_{r}(\Theta)$.

Lemma 1.1.5. Let $G$ be a unipotent, connected, affine, algebraic group over $k$. Then $\operatorname{Aut}(G)$ is an extension of an algebraic subgroup of $G L\left(G^{\mathrm{ab}}\right)$ by a connected, unipotent, affine, algebraic group. Hence the groups $G_{r}^{\mathrm{bi}}(\Theta), G_{l}^{\mathrm{bi}}(\Theta), G_{r}(\Theta), G_{l}(\Theta)$ are extensions of algebraic subgroups of $G L\left(G^{\mathrm{ab}}\right)$ by connected, unipotent, affine, algebraic groups.

Proof. Let $\mathcal{G}$ be the Lie algebra of $G$ and let $\left(\mathcal{G}^{(i)}\right)_{i}$ be a filtration of $\mathcal{G}$ by the lower central series. Any automorphism of the Lie algebra $\mathcal{G}$ preserves the filtration and the induced automorphism of $\mathcal{G}^{(i)} / \mathcal{G}^{(i+1)}$ is determined by the automorphism of $\mathcal{G}^{\text {ab }}=\mathcal{G}^{(1)} / \mathcal{G}^{(2)}$. Hence 
$\operatorname{Aut}_{\text {Lie }}(\mathcal{G})$ is an extension of a closed subgroup of $G L\left(\mathcal{G}^{\mathrm{ab}}\right)$ be a unipotent group. The lemma follows from the identification of $\operatorname{Aut}(G)$ with $\operatorname{Aut}_{\text {Lie }}(\mathcal{G})$ by the exponential map $\exp : \mathcal{G} \stackrel{\approx}{\rightarrow} G$.

Lemma 1.1.6. Assume that $G_{r}(\Theta)$ is an extension of $G_{m}$ (or $G$ such that $H^{1}(\operatorname{Gal}(\bar{k} / k), G)=$ 0 ) by a connected, unipotent, affine, algebraic group $N$. Then $T_{r}(\Theta)$ has a $k$-point.

Proof. It follows from $[\mathrm{S}]$ Proposition 4.1 that $H^{1}(\operatorname{Gal}(\bar{k} / k), N)=0$. It follow from [S] Proposition 2.2 and the assumption of the lemma that $H^{1}\left(\operatorname{Gal}(\bar{k} / k), G_{r}(\Theta)\right)=0$. Proposition 1.1 from $[\mathrm{S}]$ implies that $T_{r}(\Theta)(k) \neq \emptyset$.

Let $\Gamma^{i}(G)$ be a filtration of a group $G$ by the lower central series. Let us set $G^{(i)}:=$ $G / \Gamma^{i+1} G$. The isomorphism $\Theta: G_{1}(\mathbb{C}) \rightarrow G_{2}(\mathbb{C})$ induces isomorphisms $\Theta^{(i)}: G_{1}^{(i)}(\mathbb{C}) \rightarrow$ $G_{2}^{(i)}(\mathbb{C})$. Let $k<i$. The projections $G_{j}^{(i)} \rightarrow G_{j}^{(k)}$ for $j=1,2$ induce

$$
\begin{aligned}
& \rho_{k}^{i}: \operatorname{Iso}\left(G_{1}^{(i)}, G_{2}^{(i)}\right) \rightarrow \operatorname{Iso}\left(G_{1}^{(k)}, G_{2}^{(k)}\right), \\
& \rho(j)_{k}^{i}: \operatorname{Aut}\left(G_{j}^{(i)}\right) \rightarrow \operatorname{Aut}\left(G_{j}^{(k)}\right) \quad \text { for } \quad j=1,2 .
\end{aligned}
$$

Lemma 1.1.8. We have

i) $\overline{\rho_{k}^{i}\left(Z\left(\Theta^{(i)}\right)\right)}=Z\left(\Theta^{(k)}\right), \quad$ ii) $\rho_{k}^{i}\left(T\left(\Theta^{(i)}\right)\right)=T\left(\Theta^{(k)}\right)$,

iii) $\rho_{k}^{i}\left(T_{l}\left(\Theta^{(i)}\right)\right)=T_{l}\left(\Theta^{(k)}\right), \quad \rho_{k}^{i}\left(T_{r}\left(\Theta^{(i)}\right)\right)=T_{r}\left(\Theta^{(k)}\right)$,

iv) $\rho(1)_{k}^{i}\left(G_{l}\left(\Theta^{(i)}\right)\right)=G_{l}\left(\Theta^{(k)}\right)$,

$$
\begin{aligned}
& \rho(1)_{k}^{i}\left(G_{l}^{\mathrm{bi}}\left(\Theta^{(i)}\right)\right)=G_{l}^{\mathrm{bi}}\left(\Theta^{(k)}\right), \\
& \rho(2)_{k}^{i}\left(G_{r}^{(\text {resp. } b i)}\left(\Theta^{(i)}\right)\right)=G_{r}^{(\text {resp. } b i)}\left(\Theta^{(k)}\right) .
\end{aligned}
$$

Proof. In the point i) $\overline{(\quad)}$ means the Zariski closure and we skipped its proof because we do not need this fact later.

Let us set $p=\rho_{k}^{i}$ and $p^{\prime}=\rho(2)_{k}^{i}$. Observe that the image $p^{\prime}\left(G_{r}\left(\Theta^{(i)}\right)\right)$ of the group $G_{r}\left(\Theta^{(i)}\right)$ by the morphism $p^{\prime}$ is a closed subgroup of $\operatorname{Aut}\left(G_{2}^{(k)}\right)$ defined over $k$. This implies that $p\left(T_{r}\left(\Theta^{(i)}\right)\right)$ is a closed subvariety of $\operatorname{Iso}\left(G_{1}^{(k)}, G_{2}^{(k)}\right)$ and a $p^{\prime}\left(G_{r}\left(\Theta^{(i)}\right)\right)$-torsor defined over $k$. This torsor contains $\Theta^{(k)}$ as a $\mathbb{C}$-point, so we have

$$
T_{r}\left(\Theta^{(k)}\right) \subset p^{\prime}\left(T_{r}\left(\Theta^{(i)}\right)\right) \text { and } \quad G_{r}\left(\Theta^{(k)}\right) \subset p^{\prime}\left(G_{r}\left(\Theta^{(i)}\right)\right) .
$$


Let $P\left(\right.$ resp. $\left.P^{\prime}\right)$ be the projection $p\left(\right.$ resp. $\left.p^{\prime}\right)$ restricted to $T_{r}\left(\Theta^{(i)}\right)\left(\operatorname{resp} . G_{r}\left(\Theta^{(i)}\right)\right)$. Then $P^{-1}\left(T_{r}\left(\Theta^{(k)}\right)\right)$ is $P^{\prime-1}\left(G_{r}\left(\Theta^{(k)}\right)\right)$-torsor defined over $k$, which contain $\Theta^{(i)}$ as a $\mathbb{C}$-point. Hence we get $P^{-1}\left(T_{r}\left(\Theta^{(k)}\right)\right)=T_{r}\left(\Theta^{(i)}\right)$ and $P^{\prime-1}\left(G_{r}\left(\Theta^{(k)}\right)\right)=G_{r}\left(\Theta^{(i)}\right)$ This implies that $p\left(T_{r}\left(\Theta^{(i)}\right)\right)=T_{r}\left(\Theta^{(k)}\right)$ and $p^{\prime}\left(G_{r}\left(\Theta^{(i)}\right)\right)=G_{r}\left(\Theta^{(k)}\right)$. All other statements are proved in the same way.

\subsection{Affine, pro-algebraic, pro-unipotent groups and torsors.}

1.2.1 We assume that $G=\underset{i}{\lim } G^{(i)}$ where the groups $G^{(i)}$ are affine, connected, unipotent, algebraic groups over $k$. We assume further that $G^{(i)}=G / \Gamma^{i+1} G$. Finally we assume that the Lie algebra $\mathcal{G}$ of $G$ is finitely presented i.e. for $i$ big enough the number of relations, defining $\mathcal{G} / \Gamma^{i+1} \mathcal{G}$, of degree less than $i+1$ does not depend on $i$.

1.2.2. The condition that $G$ is finitely presented implies that for $i$ big enough the morphisms

$$
\operatorname{Aut}\left(G^{(i+1)}\right) \rightarrow \operatorname{Aut}\left(G^{(i)}\right)
$$

are surjective. We set

$$
\operatorname{Aut}(G):={\underset{i}{\longleftarrow} \lim }_{\operatorname{lnt}} \operatorname{uut}\left(G^{(i)}\right)
$$

Similarly, if $G_{1}$ and $G_{2}$ satisfy 1.2 .1 and if there is an isomorphism $G_{1, L} \rightarrow G_{2, L}$ for some extension $L$ of $k$, then the morphisms

$$
\operatorname{Iso}\left(G_{1}^{(i+1)}, G_{2}^{(i+1)}\right) \rightarrow \operatorname{Iso}\left(G_{1}^{(i)}, G_{2}^{(i)}\right)
$$

are surjective for $i$ big enough. We set

$$
\operatorname{Iso}\left(G_{1}, G_{2}\right):=\underbrace{\lim }_{i} \operatorname{Iso}\left(G_{1}^{(i)}, G_{2}^{(i)}\right)
$$

Observe that $\operatorname{Iso}\left(G_{1}, G_{2}\right)$ is an $\left(\operatorname{Aut}\left(G_{1}\right), \operatorname{Aut}\left(G_{2}\right)\right)$-bitorsor defined over $k$.

1.2.3. Examples of groups satisfying 1.2 .1 . 
1) Let $G$ be a finitely presented group, for example $G=\pi_{1}(X, x)$ where $X$ is a complex algebraic variety. Let $\operatorname{Lie}(G):=\bigoplus_{n=1}^{\infty}\left(\Gamma^{n} G / \Gamma^{n+1} G\right) \otimes \mathbb{Q}$, let $L(G):=\overleftarrow{i}_{i}^{\lim } \operatorname{Lie}(G) / \Gamma^{i} \operatorname{Lie}(G)$ be a completed Lie algebra with respect to the lower central series and let $\pi(G)$ be $L(G)$ equipped with the multiplication given by the Baker-Campbell-Hausdorff formula. Then there is an affine group scheme $\Pi(G)$ over $\mathbb{Q}$ satisfying 1.2 .1 such that $\Pi(G)(\mathbb{Q})=\pi(G)$. 2) $G=\operatorname{Spec}\left(H_{\mathrm{DR}}^{0}\left((V, x)^{\bullet}\right)\right)$ where $V$ is a smooth, algebraic variety over $k$.

Lemma 1.2.4. Let $G$ be as in 1.2.1. The affine group scheme $\operatorname{Aut}(G)$ is an extension of a closed subgroup of $G L\left(G^{\mathrm{ab}}\right)$ by an affine, connected, pro-unipotent, pro-algebraic group.

The lemma follows from Lemma 1.1.5 and the definition of $\operatorname{Aut}(G)$.

1.2.5 Let $G_{1}$ and $G_{2}$ satisfy 1.2.1. Let $\Theta: G_{1}(\mathbb{C}) \rightarrow G_{2}(\mathbb{C})$ be an isomorphism. Then $\Theta=\lim _{i} \Theta^{(i)}$ where $\Theta^{(i)}: G_{1}^{(i)}(\mathbb{C}) \rightarrow G_{2}^{(i)}(\mathbb{C})$ are isomorphisms.

Let us set

$$
\begin{aligned}
& Z(\Theta):=\underbrace{\lim }_{i} Z\left(\Theta^{(i)}\right) \\
& T(\Theta):=\underbrace{\lim _{i}}_{i} T\left(\Theta^{(i)}\right), \quad G_{r}^{\mathrm{bi}}(\Theta):=\underbrace{\lim _{i}}_{i} G_{r}^{\mathrm{bi}}\left(\Theta^{(i)}\right), \\
& G_{l}^{\mathrm{bi}}(\Theta):={\underset{\lim }{\leftarrow}}_{i} G_{l}^{\mathrm{bi}}\left(\Theta^{(i)}\right), \\
& T_{r}(\Theta):=\lim _{i} T_{r}\left(\Theta^{(i)}\right), \quad G_{r}(\Theta):={\underset{\lim }{i}}_{i} G_{r}\left(\Theta^{(i)}\right) ; \\
& T_{l}(\Theta):={\underset{\iota}{\leftarrow}}_{i} T_{l}\left(\Theta^{(i)}\right), \quad G_{l}(\Theta):=\underbrace{\lim }_{i} G_{l}\left(\Theta^{(i)}\right) .
\end{aligned}
$$

Then $T(\Theta)$ is a $\left(G_{l}^{\mathrm{bi}}(\Theta), G_{r}^{\mathrm{bi}}(\Theta)\right)$-bitorsor, $T_{r}(\Theta)$ is a right $G_{r}(\Theta)$-torsor and $T_{l}(\Theta)$ is a left $G_{l}(\Theta)$-torsor.

Lemma 1.2.6. If for each $i T_{r}\left(\Theta^{(i)}\right)(k) \neq \emptyset\left(\right.$ resp. $T_{l}\left(\Theta^{(i)}\right) \neq \emptyset$, resp. $\left.T\left(\Theta^{(i)}\right) \neq \emptyset\right)$ then $T_{r}(\Theta)(k) \neq \emptyset\left(\right.$ resp. $T_{l}(\Theta) \neq \emptyset$, resp. $\left.T(\Theta) \neq \emptyset\right)$.

The lemma follows from the fact that the morphisms $T_{r}\left(\Theta^{(i+1)}\right)(k) \rightarrow T_{r}\left(\Theta^{(i)}\right)(k)$ are surjective for $i$ big enough by 1.2.2. 


\section{$\S 2$. Torsors associated to non-abelian unipotent periods}

Let $X$ be a smooth, quasi-projective algebraic variety defined over the field $k$ of characteristic zero. Assume that $X$ has a $k$-point $x$. Let us fix an embedding $k \hookrightarrow \mathbb{C}$. In [W1] we have constructed connected, affine, pro-algebraic, pro-unipotent, finitely presented group schemes $\pi_{1}^{\mathrm{B}}(X(\mathbb{C}), x)$ and $\pi_{1}^{\mathrm{DR}}(X, x)$ over Spec $\mathbb{Q}$ and Spec $k$ respectively.

We set

$$
\begin{aligned}
& \pi_{1}^{\mathrm{B}}(X(\mathbb{C}), x)^{n}:=\pi_{1}^{\mathrm{B}}(X(\mathbb{C}), x) / \Gamma^{n+1} \pi_{1}^{\mathrm{B}}(X(\mathbb{C}), x) ; \\
& \pi_{1}^{\mathrm{DR}}(X, x)^{n}:=\pi_{1}^{\mathrm{DR}}(X, x) / \Gamma^{n+1} \pi_{1}^{\mathrm{DR}}(X, x) .
\end{aligned}
$$

Then we have:

$$
\pi_{1}^{\mathrm{B}}(X(\mathbb{C}), x)={\underset{\varliminf}{n}}_{\lim } \pi_{1}^{\mathrm{B}}(X(\mathbb{C}), x)^{n}, \pi_{1}^{\mathrm{DR}}(X, x)=\underbrace{\lim _{n}}_{n} \pi_{1}^{\mathrm{DR}}(X, x)^{n} .
$$

In [W1] Proposition 7.5 we have also constructed a homomorphism (called $B \circ \alpha$ in [W1])

$$
\mathcal{C}_{x}: \pi_{1}^{\mathrm{B}}(X(\mathbb{C}), x)(\mathbb{Q}) \rightarrow \pi_{1}^{\mathrm{DR}}(X, x)(\mathbb{C})
$$

such that the induced map on $\mathbb{C}$-points, also denoted by $\mathcal{C}_{x}$,

$$
\mathcal{C}_{x}: \pi_{1}^{\mathrm{B}}(X(\mathbb{C}), x)(\mathbb{C}) \rightarrow \pi_{1}^{\mathrm{DR}}(X, x)(\mathbb{C})
$$

is an isomorphism. $\mathcal{C}_{x}$

We have $\mathcal{C}_{x}={\underset{\lim }{n}}_{\mathcal{C}_{x}^{n}}^{n}$, where $\mathcal{C}_{x}^{n}: \pi_{1}^{\mathrm{B}}(X(\mathbb{C}), x)^{n}(\mathbb{C}) \rightarrow \pi_{1}^{\mathrm{DR}}(X, x)^{n}(\mathbb{C})$ is induced by

For each $n$ we have an $\left(\operatorname{Aut}\left(\pi_{1}^{\mathrm{B}}(X(\mathbb{C}), x)^{n} \times k\right) ; \operatorname{Aut}\left(\pi_{1}^{\mathrm{DR}}(X, x)^{n}\right)\right)$-bitorsor $\mathrm{Iso}^{n}:=$ Iso $\left(\pi_{1}^{\mathrm{B}}(X(\mathbb{C}), x)^{n} \times k ; \pi_{1}^{\mathrm{DR}}(X, x)^{n}\right)$. We have also a (left) $\operatorname{Aut}\left(\pi_{1}^{\mathrm{B}}(X(\mathbb{C}), x)^{n} \times k\right)$-torsor $\mathrm{Iso}^{n}$ and a (right) $\operatorname{Aut}\left(\pi_{1}^{\mathrm{DR}}(X, x)^{n}\right)$-torsor $\mathrm{Iso}^{n}$.

We shall concentrate mainly on the (right) $\operatorname{Aut}\left(\pi_{1}^{\mathrm{DR}}(X, x)^{n}\right)$-torsor Iso ${ }^{n}$.

Applying the construction from Section 1 to the isomorphism $\mathcal{C}_{x}^{n}$ we get the $\mathrm{G}_{r}\left(\mathcal{C}_{x}^{n}\right)$ torsor $\mathrm{T}_{r}\left(\mathcal{C}_{x}^{n}\right)$ over Spec $k$. The group $\mathrm{G}_{r}\left(\mathcal{C}_{x}^{n}\right)$ is a subgroup of $\operatorname{Aut}\left(\pi_{1}^{\mathrm{DR}}(X, x)^{n}\right)$. 
We set

$$
\mathrm{G}_{r}\left(\mathcal{C}_{x}\right):=\lim _{n} \mathrm{G}_{r}\left(\mathcal{C}_{x}^{n}\right)
$$

and

$$
\mathrm{T}_{r}\left(\mathcal{C}_{x}\right):=\lim _{n} \mathrm{~T}_{r}\left(\mathcal{C}_{x}^{n}\right)
$$

We have projections

$$
p_{n}: \operatorname{Aut}\left(\pi_{1}^{\mathrm{DR}}(X, x)^{n}\right) \rightarrow \operatorname{Out}\left(\pi_{1}^{\mathrm{DR}}(X, x)^{n}\right):=\operatorname{Aut}(\cdots) / \operatorname{Inn}(\cdots)
$$

and

$$
p: \operatorname{Aut}\left(\pi_{1}^{\mathrm{DR}}(X, x)\right) \rightarrow \operatorname{Out}\left(\pi_{1}^{\mathrm{DR}}(X, x)\right)
$$

Definition 2.1. i) The group $\mathcal{G}(X, x)^{(n)}$ is the image of $\mathrm{G}_{r}\left(\mathcal{C}_{x}^{n}\right)$ in $\operatorname{Out}\left(\pi_{1}^{\mathrm{DR}}(X, x)^{n}\right)$.

ii) The group $\mathcal{G}(X, x)$ is the image of $\mathrm{G}_{r}\left(\mathcal{C}_{x}\right)$ in $\operatorname{Out}\left(\pi_{1}^{\mathrm{DR}}(X, x)^{n}\right)$.

Let $x$ and $y$ be two $k$-points of $X$. Let $\gamma$ be a path in $X(\mathbb{C})$ from $x$ to $y$. Then $\gamma$ induces an isomorphism $c_{\gamma}: \pi_{1}^{\mathrm{DR}}\left(X_{\mathbb{C}}, x\right) \rightarrow \pi_{1}^{\mathrm{DR}}\left(X_{\mathbb{C}}, y\right)$. The induced isomorphisms of outer automorphisms groups

$$
\left(c_{\gamma}\right)_{*}: \operatorname{Out}\left(\pi_{1}^{\mathrm{DR}}\left(X_{\mathbb{C}}, x\right)\right) \rightarrow \operatorname{Out}\left(\pi_{1}^{\mathrm{DR}}\left(X_{\mathbb{C}}, y\right)\right)
$$

does not depend on the choice of $\gamma$ and gives the canonical identification. We need this identification over Spec $k$.

Let us consider the morphism $p^{\bullet}: X^{\Delta[1]} \rightarrow X^{\partial \Delta[1]}$ of cosimplicial spaces. Let us set $(X ; x, y)^{\bullet}:=p^{\bullet-1}(x, y)$. Then $\operatorname{Spec}\left(H_{\mathrm{DR}}^{0}\left((X ; x, y)^{\bullet}\right)\right)$ is a left $\pi_{1}^{\mathrm{DR}}(X, x)$-torsor and a right $\pi_{1}^{\mathrm{DR}}(X, y)$-torsor (see [W1] Section 3). It follows from [S] Proposition 4.1 that any torsor over $\pi_{1}^{\mathrm{DR}}(X, x)^{n}$ is trivial, hence any torsor over $\pi_{1}^{\mathrm{DR}}(X, x)$ is trivial (inverse limit of surjective maps of sets ( $k$-points) is always non empty). Any $k$-point $\eta$ of $\operatorname{Spec}\left(H_{\mathrm{DR}}^{0}\left((X ; x, y)^{\bullet}\right)\right)$ determines on isomorphism

$$
c_{\eta}: \pi_{1}^{\mathrm{DR}}(X, x) \rightarrow \pi_{1}^{\mathrm{DR}}(X, y)
$$


The isomorphism is unique up to conjugation by elements of $\pi_{1}^{\mathrm{DR}}(X, x)$. Hence the induced isomorphism

$$
\left(c_{\eta}\right)_{*}: \operatorname{Out}\left(\pi_{1}^{\mathrm{DR}}(X, x)\right) \rightarrow \operatorname{Out}\left(\pi_{1}^{\mathrm{DR}}(X, y)\right)
$$

is canonical (does not depend on the choice of a $k$-point of $\left.\operatorname{Spec}\left(H_{\mathrm{DR}}^{0}\left((X ; x, y)^{\bullet}\right)\right)\right)$.

The monodromy representations $\mathcal{C}_{x}$ and $\mathcal{C}_{y}$ are related by the following commutative diagram

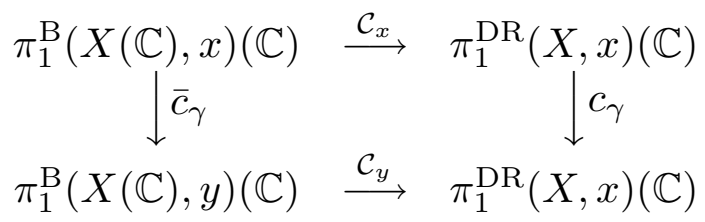

where $\bar{c}_{\gamma}$ and $c_{\gamma}$ are induced by the path $\gamma$. Observe that $c_{\gamma}=c_{\eta} \circ \operatorname{conj}(g)$, where $\eta$ is a $k$-point of $\operatorname{Spec}\left(H_{\mathrm{DR}}^{0}((X ; x, y))\right)$ and $\operatorname{conj}(g)$ is a conjugation by an element $g \in$ $\pi_{1}^{\mathrm{DR}}(X, x)(\mathbb{C})$. This implies the following result.

Proposition 2.2. The groups $\mathcal{G}(X, x)^{n}$ and $\mathcal{G}(X, y)^{(n)}$ coincides under the canonical isomorphism $\left(c_{\eta}\right)_{*}$. The groups $\mathcal{G}(X, x)$ and $\mathcal{G}(X, y)$ coincides under the canonical isomorphism $\left(c_{\eta}\right)_{*}$. We shall denote these groups by $\mathcal{G}_{\mathrm{DR}}(X)^{(n)}$ and $\mathcal{G}_{\mathrm{DR}}(X)$ respectively.

Some calculations and results considers in the sequel seems to lead to the following conjectures.

Let $\mathcal{C}_{k}: G_{k}:=\operatorname{Gal}(\bar{k} / k) \rightarrow \operatorname{Out}\left(\pi_{1}(X \underset{k}{\times} \bar{k}, x)_{l}\right)$ be the natural representation of the Galois groups. Let $\mathcal{C}_{k}^{n}: G_{k} \rightarrow \operatorname{Out}\left(\pi_{1}(X \underset{k}{\times \bar{k}}, x)_{l} / \Gamma^{n+1}(\cdots)\right)$ be induced by $\mathcal{C}_{k}$.

If $G$ is a group (algebraic or $p$-adic analytic) we denote by $\operatorname{Lie}(G)$ its Lie algebra.

Conjecture 2.3. For each $n$ there exists a Lie algebra $\mathcal{G}^{(n)}$ over $\mathbb{Q}$ such that

i) $\mathbb{Q} \otimes \operatorname{Lie}\left(\mathcal{C}_{k}^{n}\left(G_{k}\right)\right) \approx \mathcal{G}^{(n)} \otimes \mathbb{Q}_{l}$,

ii) $\mathbb{Q} \otimes \operatorname{Lie}\left(\mathcal{G}_{\mathrm{DR}}(X)^{(n)}\right) \approx \mathcal{G}^{(n)} \otimes k$.

The Lie algebras $\mathcal{G}^{(n)}$ form an inverse system. Let us set $\mathcal{G}=\underset{n}{\lim _{n}} \mathcal{G}^{n}$. Then

i) $\mathbb{Q} \otimes \operatorname{Lie}\left(\mathcal{C}_{k}\left(G_{k}\right)\right) \approx \mathcal{G} \otimes \mathbb{Q}_{l}$,

ii) $\mathbb{Q} \otimes \operatorname{Lie}\left(\mathcal{G}_{\mathrm{DR}}(X)\right) \approx \mathcal{G} \otimes k$. 


\section{$\S 3$. Canonical connection with logarithmic singularities.}

Let $X$ be a smooth, projective scheme of finite type over a field $k$ of characteristic zero. Let $D$ be a divisor with normal crossings in $X$ and let $V=X \backslash D$. Let

$$
A^{*}(V):=\Gamma\left(X, \Omega_{X}^{*}\langle\log D\rangle\right)
$$

be a differential algebra of global sections of the algebraic De Rham complex on $X$ with logarithmic singularities along $D$.

3.1. It follows from [D1] Corollaire 3.2.14 that each element of $A^{*}(V)$ is closed and the natural map $A^{*}(V) \rightarrow H_{D R}^{*}(V)$ is injective.

We shall denote by $\wedge^{2}\left(A^{1}(V)\right)$ the exterior product of the vector space $A^{1}(V)$ with itself and by $A^{1}(V) \wedge A^{1}(V)$ the image of $\wedge^{2}\left(A^{1}(V)\right)$ in $A^{2}(V)$.

Let $H(V):=\left(A^{1}(V)\right)^{*}$ and $R(V):=\left(A^{1}(V) \wedge A^{1}(V)\right)^{*}$ be dual vector spaces. The map $\wedge^{2}\left(A^{1}(V)\right) \rightarrow A^{1}(V) \wedge A^{1}(V)$ induces a map $R(V) \rightarrow \Lambda^{2}(H(V))$.

Let Lie $(H(V))$ be a free Lie algebra over $k$ on $H(V)$. Observe that $R(V)$ is contained in degree 2 terms of Lie $(H(V))$. Let $(R(V))$ be a Lie ideal generated by $R(V)$. We set

$$
\operatorname{Lie}(V):=\operatorname{Lie}(H(V)) /(R(V))
$$

and

$$
L(V):={\underset{\varliminf}{n}}_{\lim _{n}}\left(\operatorname{Lie}(V) / \Gamma^{n} \operatorname{Lie}(V)\right) .
$$

The Lie algebra $L(V)$ we equip with the multiplication given by the Baker-CampbellHausdorff formula and the obtained group we shall denote by $\pi(V)$. Its Lie algebra can be identified with $L(V)$. We define a one form $\omega_{V}$ on $V$ with values in the Lie algebra $L(V)$ in the following way. The form $\omega_{V}$ corresponds to the identity homomorphism id $A^{1}(V)$ under the natural isomorphism

$$
\left.A^{1}(V) \otimes H(V)=A^{1}(V) \otimes\left(A^{1}(V)\right)^{*} \approx \operatorname{Hom}\left(A^{1}(V), A^{1}(V)\right)\right) .
$$


Lemma 3.2. The one-form $\omega_{V}$ is integrable.

Proof. It is sufficient to show that $d \omega_{V}+\frac{1}{2}\left[\omega_{V}, \omega_{V}\right]=0$. It follows from 3.1 that $d \omega_{V}=0$. We have exact sequences

$$
0 \rightarrow K \longmapsto \wedge^{2}\left(A^{1}(V)\right) \rightarrow A^{1}(V) \wedge A^{1}(V) \rightarrow 0
$$

and

$$
0 \leftarrow K^{*} \leftarrow \wedge^{2}(H(V)) \leftarrow R(V) \leftarrow 0 .
$$

The two-form $\left[\omega_{V}, \omega_{V}\right] \in A^{1}(V) \wedge A^{1}(V) \otimes \wedge^{2} H(V) / R(V) \approx\left(\wedge^{2}\left(A^{1}(V)\right) / K\right) \otimes K^{*}$ is represented by a map $K \rightarrow \wedge^{2}\left(A^{1}(V)\right) \rightarrow A^{1}(V) \wedge A^{1}(V)$, hence it is zero.

Let $T[H(V)]$ be a tensor algebra over $k$ on $H(V)$ and let $(R(V))$ be an ideal of $T[H(V)]$ generated by $R(V)$. Let $\mathbb{Q}(V):=T[H(V)] / R(V)$ be the quotient algebra and let $\widehat{\mathbb{Q}}(V)$ be its completion with repect to the augmentation ideal $I:=\operatorname{ker}(\mathbb{Q}(V) \rightarrow k)$, i.e. $\widehat{\mathbb{Q}}(V):=\underbrace{}_{n} \lim _{n}\left(\mathbb{Q}(V) / I^{n}\right)$. Let $P(V)$ be the group of invertible elements in $\widehat{\mathbb{Q}}(V)$, whose constant terms are equal 1.

The elements of $L(V)$ we identify with Lie elements (can be of infinite length) in $P(V)$. The exponential series defines an injective homomorphism

$$
\exp : \pi(V) \rightarrow P(V)
$$

The inverse of exp is defined on the subgroup $\exp (\pi(V))$ of $P(V)$ and it is given by the formula

$$
\log z=(z-1)-1 / 2(z-1)^{2}+1 / 3(z-1)^{3}-1 / 4(z-1)^{4}+\ldots
$$

Let us assume that $k$ is the field of complex numbers $\mathbb{C}$. Then $V$ is a complex variety with the standard complex topology.

Let $x, z \in V$ be two points in $V$ and let $\gamma$ be a smooth path in $V$ from $x$ to $z$.

Let $\Lambda_{V}(z ; x, \gamma)$ (resp. $\left.L_{V}(z ; x, \gamma)\right)$ be a horizontal section along $\gamma$ of the principal $P(V)$ (resp. $\pi(V)$ )-bundle

$$
V \times P(V) \rightarrow V \quad(\text { resp. } V \times \pi(V) \rightarrow V)
$$


equipped with the connection given by $\omega_{V}$ and such that $\Lambda_{V}(x ; x, \gamma)=1\left(\operatorname{resp} . L_{V}(x ; x, \gamma)=\right.$ $0)$.

Definition 3.3. Let $x \in V$ and let $\alpha \in \pi_{1}(V, x)$ be a loop. We shall define a homomorphism

$$
\theta_{x, V}: \pi_{1}(V, x) \rightarrow P(V) \quad\left(\text { resp. } \theta_{x, V}: \pi_{1}(V, x) \rightarrow \pi(V)\right)
$$

by the formula

$$
\theta_{x, V}(\alpha):=\Lambda_{V}(\alpha(1) ; x, \alpha) \quad\left(\operatorname{resp} . \theta_{x, V}(\alpha):=L_{V}(\alpha(1) ; x, \alpha)\right)
$$

and we call it the monodromy homomorphism of the form $\omega_{V}$ (at the point $x$ ).

Proposition 3.4. Let $x_{1}, x_{2} \in V$. Then the monodromy homomorphisms $\theta_{x_{1}, V}$ and $\theta_{x_{2}, V}$ of the form $\omega_{V}$ are conjugated.

Proof. It is a property of a connection on a principal fiber bundle.

Proposition 3.5. Assume that

$$
A^{1}(V) \rightarrow H_{D R}^{1}(V)
$$

is an isomorphism. Then the Lie algebra Lie $(V)$ is isomorphic to the Lie algebra of the fundamental group of $V$.

Proof. Let $\Omega^{*}(V)$ be a differential algebra of complex valued, global, smooth, differential forms on $V$. The inclusion $A^{*}(V) \rightarrow \Omega^{*}(V)$ induces an isomorphism of the "stage one" minimal models. Moreover the "stage one" minimal model of $A^{*}(V)$ is formal as $d_{1}=$ $d_{2}=0$. This implies the statement of the proposition.

Proposition 3.6. If $A^{1}(V) \rightarrow H_{D R}^{1}(V)$ is an isomorphism then the monodromy homomorphism $\theta_{x, V}: \pi_{1}(V, x) \rightarrow \pi(V)$ induces an isomorphism of the Malcev $\mathbb{C}$-completion of $\pi_{1}(V, x)$ into $\pi(V)$.

Proof. The Sullivan theory of minimal models recovers the Malcev $\mathbb{Q}$-completion $\pi_{1}(V, x)_{0}(\mathbb{Q})$ of $\pi_{1}(V, x)$. (See $\S$ A.1 of Appendix for the notation $\pi_{1}(V, x)_{0}$.) The formality of the "state one" minimal model of $V$ implies that the Malcev $\mathbb{C}$-completion of $\pi_{1}(V, x)$ is (isomorphic 
to) $\pi(V)$. Hence the groups $\pi_{1}(V, x)_{0}(\mathbb{C})$ and $\pi(V)$ are isomorphic. By the universal property of the Malcev $\mathbb{C}$-completion there is an isomorphism $\bar{\theta}_{x, V}: \pi_{1}(V, x)_{0}(\mathbb{C}) \stackrel{\approx}{\longrightarrow} \pi(V)$ of affine, pro-nilpotent groups such that the diagram

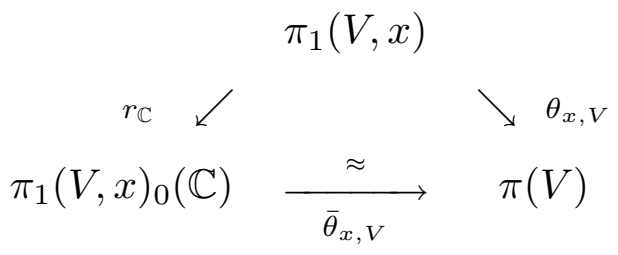

commutes.

Let $X_{i}$ (for $i=1,2$ ) be smooth, projective schemes of finite type over $k$. Let $D_{i}$ be divisors with normal crossings in $X_{i}$ (for $i=1,2$ ). Let $V_{i}=X_{i} \backslash D_{i}$ (for $i=1,2$ ) and let $f: X_{1} \rightarrow X_{2}$ be a morphism such that $f^{-1}\left(D_{2}\right)=D_{1}$. Then $f$ induces $f^{*}: A^{1}\left(V_{2}\right) \rightarrow$ $A^{1}\left(V_{1}\right)$.

Let $f_{*}: H\left(V_{1}\right) \rightarrow H\left(V_{2}\right)$ be the dual map. This map induces group homomorphisms

$$
f_{*}: P\left(V_{1}\right) \rightarrow P\left(V_{2}\right)
$$

and

$$
f_{*}: \pi\left(V_{1}\right) \rightarrow \pi\left(V_{2}\right)
$$

Lemma 3.7. We have

$$
f_{*}\left(\omega_{V_{1}}\right)=f^{*}\left(\omega_{V_{2}}\right)
$$

Corollary 3.8. We have

$$
f_{*}\left(\Lambda_{V_{1}}(z ; x, \gamma)\right)=\Lambda_{V_{2}}(f(z) ; f(x), f(\gamma)) .
$$

and

$$
f_{*}\left(L_{V_{1}}(z ; x, \gamma)\right)=L_{V_{2}}(f(z) ; f(x), f(\gamma)) .
$$

The lemma follows from the definition of $\omega_{V_{i}}$ as id $A^{1}\left(V_{i}\right)$.

Let $\omega_{1}, \ldots, \omega_{n}$ be a base of $A^{1}(V)$. Let $X_{1}, \ldots, X_{n}$ be a dual base of $H(V)$. Then $P(V)$ is a multiplicative group of the algebra of formal power series in non-commuting variables $X_{1}, \ldots, X_{n}$ divided by the ideal generated by $R(V)$.

If $\alpha\left(X_{1}, \ldots, X_{n}\right)$ is a formal power series in non-commuting variables $X_{1}, \ldots, X_{n}$ we shall denote by ' $\alpha\left(X_{1}, \ldots, X_{n}\right)$ its image in $P(V)$. 
Proposition 3.9. We have

i) $\left(z, \Lambda_{V}(z ; x, \gamma)\right)=\left(z,{ }^{\prime} 1+\sum\left((-1)^{k} \int_{x, \gamma}^{z} \omega_{i_{1}}, \ldots, \omega_{i_{k}}\right) X_{i_{k}} \cdot \ldots \cdot X_{i_{1}}\right) \in V \times P(V)$;

(the summation is over all non-commutative monomials in variables $X_{1}, \ldots, X_{n}$, the interated integrals are calculated along the path $\gamma$ )

ii) $\quad L_{V}(z ; x, \gamma)=\log \left(\Lambda_{V}(z ; x, \gamma)\right)$

Proof. The principal bundle $V \times \mathbb{C}\left\{\left\{X_{1}, \ldots, X_{n}\right\}\right\}^{*} \rightarrow V$ equipped with the connection given by $\sum_{i=1}^{n} \omega_{i} \otimes X_{i}$ has horizontal sections given by

$$
z \rightarrow\left(z, 1+\sum\left((-1)^{k} \int_{x, \gamma}^{z} \omega_{i_{1}}, \ldots, \omega_{i_{k}}\right) X_{i_{k}} \cdot \ldots \cdot X_{i_{1}}\right)
$$

Hence the point i) follows. Observe that $\exp : \pi(V) \rightarrow P(V)$ identifies $\omega_{V} \in A^{1}(V) \otimes$ Lie $(\pi(V))$ with $\omega_{V} \in A^{1}(V) \otimes \operatorname{Lie}(P(V))$. Hence the point ii) follows.

\section{$\S 4$. The Gauss-Manin connection associated with the morphism $X^{\Delta[1]} \rightarrow X^{\partial \Delta[1]}$ of cosimplicial schemes.}

4.0. Let $V$ be a smooth, quasi-projective scheme over a field $k$ of characteristic zero. The inclusion of simplicial sets

$$
\partial \Delta[1] \hookrightarrow \Delta[1]
$$

induces a morphism of cosimplicial schemes

$$
\rho^{\bullet}: V^{\Delta[1]} \rightarrow V^{\partial \Delta[1]}
$$

Imitating the construction of Katz and Oda (see [KO]) we equipped the sheaves $H^{i}\left(t R_{\rho_{*}^{\bullet}} \Omega_{\left(V^{\Delta[1]}\right) /\left(V^{\partial \Delta[1]}\right)}^{*}\right)$ with the integrable connection $d_{k}$ (see [W1]). We recall that $(V, x)^{\bullet}=\rho^{\bullet-1}(x, x)$.

4.1. Let $X$ be a smooth, projective variety defined over $\mathbb{C}$. Let $D$ be a divisor with normal crossings in $X$ and let $V=X \backslash D$. Let $\Omega_{\langle D\rangle}^{1}(X)$ be global, meromorphic one forms on $X$ with logarithmic singularities along $D$. We shall assume that $\Omega_{\langle D\rangle}^{1}(X) \approx$ 
$H_{D R}^{1}(V)$. We shall calculate the monodromy representation of the connection $d_{\mathbb{C}}$ on the sheaf $H^{0}\left(t R_{\rho_{*}^{*}} \Omega_{\left(V^{\Delta[1]}\right) /\left(V^{\partial \Delta[1]}\right)}^{*}\right)$. Let

$$
L(V)^{*}:=\underset{n}{\lim }\left(\operatorname{Lie}(V) / \Gamma^{n} \operatorname{Lie}(V)\right)^{*} .
$$

Then the algebra of polynomial functions on $\pi(V)$ coincides with the symmetric algebra on $L(V)^{*}$ i.e.

$$
\operatorname{Alg}(\pi(V))=S\left(L(V)^{*}\right)
$$

The group $\pi(V)$ acts on $\operatorname{Alg}(\pi(V))$ on the left by the formula
a) $g(f)(x):=f(x \cdot g)$
b) $g(f)(x):=f\left(g^{-1} x\right)$

We form the associated vector bundle

$$
V \times \operatorname{Alg}(\pi(V)) \approx V \times \pi(V) \underset{\pi(V)}{\times} \operatorname{Alg}(\pi(V)) \rightarrow V
$$

and we equipped it with the connection induced by $\omega_{V}$. This connection we shall denote by $\omega_{V}^{\prime}$ in the case a) and by ${ }^{\prime} \omega_{V}$ in the case b).

Lemma 4.1. The monodromy representation of the associated vector bundle is given by

$$
\pi_{1}(V, x) \ni \gamma \rightarrow\left(f \rightarrow \theta_{x}(\gamma)(f)\right) \in \operatorname{Aut}(\operatorname{Alg}(\pi(V)))
$$

Proof. This follows from the definition of an associated vector bundle.

4.2. Let us set $A(V):=\Omega_{\langle D\rangle}^{1}(X)$ and $A^{2}(V):=A(V) \wedge A(V) \subset \Omega_{\langle D\rangle}^{2}(X)$. The inclusion of complexes of differential graded algebras

$$
\left.(\mathbb{C} \stackrel{0}{\longrightarrow} A(V)) \stackrel{0}{\longrightarrow} A^{2}(V)\right) \hookrightarrow \Omega^{*}(V) .
$$

induces an isomorphism

$$
H^{0}:=H^{0}\left(\operatorname{Bar}\left(\mathbb{C} \stackrel{0}{\longrightarrow} A(V) \stackrel{0}{\longrightarrow} A^{2}(V)\right)\right) \approx H_{D R}^{0}\left((V, x)^{\bullet}\right) .
$$

Let $T\left[A(V)^{*}\right]$ be a tensor algebra on the dual vector space $A(V)^{*}$. Let $\wedge^{2}(A(V))$ be an exterior product of complex vector spaces. The surjective map $\wedge^{2}(A(V)) \rightarrow A^{2}(V)$ 
induces an inclusion $\left(A^{2}(V)\right)^{*} \hookrightarrow \wedge^{2}\left(A(V)^{*}\right)$. Let us set $R(V):=\left(A^{2}(V)\right)^{*}$. Let $(R(V))$ be an ideal in $T\left[A(V)^{*}\right]$ generated by $R(V)$ and let $\mathbb{Q}(V):=T\left[A(V)^{*}\right] /(R(V))$ be the quotient algebra. Let $I$ be its augmentation ideal and let $\mathbb{Q}(V)^{*}:=\underset{n}{\lim }\left(\mathbb{Q}(V) / I^{n}\right)^{*}$ be the direct limit of dual vector spaces.

Let us fixed a base $\omega_{1}, \ldots, \omega_{n}$ of $A(V)$. Let $X_{1}, \ldots, X_{n}$ be a dual base of $A(V)^{*}$. Then $T\left[A(V)^{*}\right]=\mathbb{C}\left\{X_{1}, \ldots, X_{n}\right\}$ is a polynomial algebra on non-commuting variables $X_{1}, \ldots, X_{n}$. Let $a\left(X_{i_{1}}, \ldots, X_{i_{k}}\right)$ denotes a linear form on $T\left[A(V)^{*}\right]$ which to $f$ associates its coefficient at $X_{i_{1}} \ldots X_{i_{k}}$. Let $T^{*}$ be a vector space generated by all $a\left(X_{i_{1}} \ldots, X_{k}\right)$ including $a(\phi)$. Observe that $\mathbb{Q}(V)^{*}$ is a subspace of $T^{*}$. We equipped $\mathbb{Q}(V)^{*}$ with a shuffle multiplication and a Hopf algebra structure given by $a\left(X_{i_{1}} \ldots, X_{i_{k}}\right) \rightarrow \sum_{l=0}^{k} a\left(X_{i_{1}} \ldots, X_{i_{l}}\right) a\left(X_{i_{l+1}}, \ldots, X_{i_{k}}\right)$.

Lemma 4.3. There is an isomorphism of Hopf algebras

$$
P: H^{0} \stackrel{\approx}{\longrightarrow} \mathbb{Q}(V)^{*}
$$

induced by $\omega_{i_{1}} \otimes \ldots \otimes \omega_{i_{k}} \rightarrow a\left(X_{i_{1}}, \ldots, X_{i_{k}}\right)$.

The symmetric algebra on the vector space $\mathbb{Q}(V)^{*}$ divided by an ideal $(a(\phi)-1)$ is the algebra of polynomial functions on $P(V)$, which we denonte by $\operatorname{Alg}(P(V))$. Let $S h(V)$ be an ideal in $\operatorname{Alg}(P(V))$ generated by shuffle product relations. Then the morphism

$$
\text { 4.4. } \mathbb{Q}: \operatorname{Alg}(P(V)) / \operatorname{Sh}(V) \stackrel{\approx}{\longrightarrow} \mathbb{Q}(V)^{*}
$$

is an isomorphism of Hopf algebras.

It follows from a theorem of R. Ree (see $[\mathrm{R}]$ Theorem 2.5) that a formal power series $f \in P(V)$ is in the image of exp : $\pi(V) \rightarrow P(V)$ if and only if its coefficients (i.e. coefficients of some lifting to $\left.\mathbb{C}\left\{\left\{X_{1}, \ldots, X_{n}\right\}\right\}\right)$ satisfy shuffle product relations. This implies that the injective homomorphism

$$
\exp : \pi(V) \rightarrow P(V)
$$

induces an isomorphism of Hopf algebras

$$
\text { 4.5. } R: \mathbb{Q}(V)^{*} \stackrel{\approx}{\longrightarrow} \operatorname{Alg}(\pi(V)) \text {. }
$$


Let $\mathcal{H}_{x}^{0}\left(\right.$ resp. $\left.{ }_{x} \mathcal{H}^{0}\right)$ be a restriction of the bundle $H^{0}\left(t R_{\rho_{*}^{*}} \Omega_{\left(V^{\Delta[1]}\right) /\left(V^{\partial \Delta[1]}\right)}^{*}\right)$ to $X \times\{x\}$ (resp. $\{x\} \times X$.) Both bundles are equipped with the induced connection, which we also denote by $d_{\mathbb{C}}$.

Our main result in this section is the following theorem.

Theorem 4.6. Let $V$ be such as in 4.1. Then the vector bundles equipped with connections

$$
\left(\mathcal{H}_{x}^{0}, d_{\mathbb{C}}\right) \quad \text { and } \quad\left(V \times \operatorname{Alg}(\pi(V)) \rightarrow V, \omega_{V}^{\prime}\right)
$$

are isomorphic in the category of algebraic vector bundles equipped with algebraic integrable connections.

$\left(\left({ }_{x} \mathcal{H}^{0}, d_{\mathbb{C}}\right)\right.$ and $\left(V \times \operatorname{Alg}(\pi(V)) \rightarrow V, \omega_{V}^{\prime}\right)$ are also isomorphic. $)$

Proof. We shall calculate horizontal sections of the connection $d_{\mathbb{C}}$ on

$\mathcal{H}:=H^{0}\left(t R \rho_{*}^{\bullet} \Omega_{\left(V^{\Delta[1]}\right) /\left(V^{\partial \Delta[1]}\right)}^{*}\right)$. We recall the construction of $d_{\mathbb{C}}$. We start with the filtration $\left\{F^{i}\right\}$ of $\Omega_{V \Delta[1]}^{*}$. The filtrations $\left\{F^{i}\right\}$ on $\Omega_{V^{n+2}}^{*}$ are defined by

$$
F^{i} \Omega_{V^{n+2}}^{*}:=\operatorname{image}\left(\Omega_{V^{n+2}}^{*-i} \otimes\left(\rho^{n}\right)^{*} \Omega_{V \times V}^{i} \rightarrow \Omega_{V^{n+2}}^{*}\right) .
$$

These filtrations give a filtration $F^{i}$ of $\Omega_{V \Delta[1]}^{*}$. The connection $d_{\mathbb{C}}$ is defined as the boundary homomorphism of the short exact sequence of complexes on $V^{\Delta[1]}$

$$
0 \rightarrow F^{1} / F^{2} \rightarrow F^{0} / F^{2} \rightarrow F^{0} / F^{1} \rightarrow 0
$$

On $V^{n+2}$ we have

i) $F^{0} / F^{1} \approx \Omega_{V^{n+2} / V \times V}^{*} \quad$ ii) $F^{0} / F^{2} \approx \Omega_{V^{n+2} / \Omega_{V^{n+2}}^{*-2}}^{*} \otimes \Omega_{V \times V}^{2}$.

Observe that $\mathcal{O}_{V} \otimes \Omega_{V^{n}}^{*} \otimes \mathcal{O}_{V} \subset F^{0} / F^{2}$.

Let us assume that $\omega=\omega_{1} \otimes \ldots \otimes \omega_{n} \in A(V)^{\otimes n}$ is closed in the total complex of $\Omega^{*}\left((V, x)^{\bullet}\right)$. Its class $[\omega]$ belongs to $H_{D R}^{0}\left((V, x)^{\bullet}\right)$ - the fiber of $\mathcal{H}$ over $(x, x) \in V \times V$.

We face two problems. We must extend $[\omega]$ to a continous section of $\mathcal{H}$ and we must show that this section is horizontal. All computations are carried in the total complex 
$\mathcal{T}:=\operatorname{Tot}\left(n \rightarrow \mathcal{O}_{V} \otimes \Omega^{*}\left(V^{n}\right) \otimes \mathcal{O}_{V}\right)$. Then $\omega_{1} \otimes \ldots \omega_{n}$ can be interpreted as a global section of $\mathcal{T}$. One verifies that the class of the element

$$
\begin{gathered}
\sum_{\substack{0 \leq k, l \leq n \\
k+l \leq n}}\left(\int_{x} \omega_{1}, \ldots, \omega_{k}\right) \otimes \omega_{k+1} \otimes \ldots \otimes \omega_{n-l} \otimes(-1)^{l}\left(\int_{x} \omega_{n}, \ldots, \omega_{n-l+1}\right) \\
\in \sum \mathcal{O}_{V} \otimes \Omega^{n-1-k}\left(V^{n-1-k}\right) \otimes \mathcal{O}_{V}
\end{gathered}
$$

is a horizontal section of $\mathcal{H}$. Hence we get.

4.7. Let $(\alpha, \beta) \in \pi_{1}(V \times V,(x, x))$. Then the representation of $\pi_{1}(V \times V,(x, x))$ is given by

$$
(\alpha, \beta): \omega_{1} \otimes \ldots \otimes \omega_{n} \rightarrow \sum_{\substack{0 \leq k, l \leq n \\ k+l \leq n}}\left(\int_{\alpha} \omega_{1}, \ldots, \omega_{k}\right) \omega_{k+1} \otimes \ldots \otimes \omega_{n-l}\left(\int_{\beta^{-1}} \omega_{n-l+1}, \ldots, \omega_{n}\right)
$$

By 4.5. the Hopf algebra $\mathbb{Q}(V)^{*}$ is isomorphic to $\operatorname{Alg}(\pi(V))$. Hence we shall consider $\omega_{V}^{\prime}$ on the associated bundle $V \times \mathbb{Q}(V)^{*} \rightarrow V$. It follows from Proposition 3.9.i) that the monodromy representation is given by

$$
\pi_{1}(V, x) \ni \alpha: a\left(X_{i_{1}}, \ldots, X_{i_{k}}\right) \rightarrow \sum_{l} a\left(X_{i_{1}}, \ldots, X_{i_{l}}\right)\left(\int_{\alpha^{-1}} \omega_{i_{l+1}}, \ldots, \omega_{i_{k}}\right)
$$

for the connection $\omega_{V}^{\prime}$, and by

$$
\pi_{1}(V, x) \ni \alpha: a\left(X_{i_{1}}, \ldots, X_{i_{k}}\right) \rightarrow \sum_{l}\left(\int_{\alpha} \omega_{i_{1}}, \ldots, \omega_{i_{l}}\right) a\left(X_{i_{l+1}}, \ldots, X_{i_{k}}\right)
$$

for the connection ' $\omega_{V}$.

The isomorphism $H^{0} \stackrel{\sim}{\rightarrow} \mathbb{Q}(V)^{*}$ defined by $\omega_{i_{1}} \otimes \ldots \otimes \omega_{i_{k}} \rightarrow a\left(X_{i_{1}}, \ldots, X_{i_{k}}\right)$ gives an isomorphism of monodromy representations of bundles $\left(\mathcal{H}_{X}, d_{\mathbb{C}}\right)$ and $\left(V \times \mathbb{Q}(V)^{*} \rightarrow V, \omega_{V}^{\prime}\right)$ (resp. $\left({ }_{x} \mathcal{H}, d_{\mathbb{C}}\right)$ and $\left.\left(V \times \mathbb{Q}(V)^{*} \rightarrow V,{ }^{\prime} \omega_{V}\right)\right)$ at the point $x \in V$.

Observe that both connections are regular. The theorem follows from [D3] Théorème 5.9. 


\section{$\S 5$. Torsors associated to the canonical unipotent connection with logarithmic singularities.}

5.0. Let $V$ be as in section 3 . We shall assume that $A^{1}(V) \rightarrow H_{D R}^{1}(V)$ is an isomorphism. We denote by $V(\mathbb{C})$ the set of complex points of $V$. In 3.1 we defined the group $\pi(V)$. It is easy to see that $\pi(V)$ is a group of $k$-points of a connected, affine, pro-unipotent, pro-algebraic group scheme over $k$. In this section we denote by $\pi(V)$ the corresponding group scheme, and by $\pi(V)(R)$ the group of $R$-points of $\pi(V)$ for a $k$-algebra $R$. Simillar notation is used for $P(V)$.

Let us define a homomorphism

$$
\theta_{x}^{-1}: \pi_{1}(V(\mathbb{C}), x) \rightarrow \pi(V)(\mathbb{C})
$$

by the formula $\left(\theta_{x}^{-1}\right)(\alpha):=\left(\theta_{x}(\alpha)\right)^{-1}$, where $\theta_{x}$ is the monodromy homomorphism of the form $\omega_{V}$.

Let us set $\pi_{0}:=\pi_{1}(V(\mathbb{C}), x)_{0}$-the Malcev $\mathbb{Q}$-completion of $\pi_{1}(V(\mathbb{C}), x)$. By the universal property of $r_{\mathbb{C}}: \pi_{1}(V(\mathbb{C}), x) \rightarrow \pi_{0}(\mathbb{C})$ (see A.1) there exists a unique homomorphism

$$
\left(\theta_{x}^{-1}\right)_{0}: \pi_{0}(\mathbb{C}) \rightarrow \pi(V)(\mathbb{C})
$$

such that $\left(\theta_{x}^{-1}\right)=\left(\theta_{x}^{-1}\right)_{0} \circ r_{\mathbb{C}}$. It follows from the Sullivan theory of minimal models that the groups (affine, pro-algebraic group schemes over $\mathbb{C}) \pi_{0}(\mathbb{C})$ and $\pi(V)(\mathbb{C})$ are isomorphic. The morphism $\left(\theta_{x}^{-1}\right)_{0}$ is an isomorphism $\bmod \Gamma^{2}$, hence it is an isomorphism.

Applying the construction from section 1.2 to the isomorphism $\left(\theta_{x}^{-1}\right)_{0}$ and to the $\operatorname{Aut}(\pi(V))$-torsor Iso $\left(\pi_{0} \times \operatorname{Spec} k, \pi(V)\right)$ we get a torsor over $k T_{r}\left(\left(\theta_{x}^{-1}\right)_{0}\right)$, and an affine, pro-algebraic group scheme over $k G_{r}\left(\left(\theta_{x}^{-1}\right)_{0}\right)$. If we work $\bmod \Gamma^{n+1}$, the map $\left(\theta_{x}^{-1}\right)_{0}$ induces $\left(\theta_{x}^{-1}\right)_{0}^{n}: \pi_{0}(\mathbb{C}) / \Gamma^{n+1} \ldots \rightarrow \pi(V)(\mathbb{C}) / \Gamma^{n+1} \ldots$ and we get a subtorsor $T_{r}\left(\left(\theta_{x}^{-1}\right)_{0}\right)^{n}$ of $\operatorname{Iso}\left(\left(\pi_{0} / \Gamma^{n+1} \pi_{0}\right) \times \operatorname{Spec} k, \pi(V) / \Gamma^{n+1} \pi(V)\right)$ and a subgroup $G_{r}\left(\left(\theta_{x}^{-1}\right)_{0}\right)^{n}$. By the definition we have $G_{r}\left(\left(\theta_{x}^{-1}\right)_{0}\right)={\underset{\lim }{n}}_{n} G_{r}\left(\left(\theta_{x}^{-1}\right)_{0}\right)^{n}$ and $T_{r}\left(\left(\theta_{x}^{-1}\right)_{0}\right)=\underset{\lim _{n}}{\lim } T_{r}\left(\left(\theta_{x}^{-1}\right)_{0}\right)^{n}$.

5.1. Let $\pi_{1}(V(\mathbb{C}), x)=\left\langle A_{1}, \ldots, A_{k} \mid r_{j}\left(A_{1}, \ldots, A_{k}\right)=0, j=1, \ldots, l\right\rangle$ be a presentation of $\pi_{1}(V(\mathbb{C}), x)$ in terms of generators and relations. We shall denote this presentation by $\left.P\left(A_{i}\right)\right)$. For simplicity let us set $P=P\left(\left(A_{i}\right)\right)$. 
Notation. $\left\langle w_{1}, \ldots, w_{n}\right\rangle$ is a vector subspace generated by vectors $w_{1}, \ldots, w_{n} ; \bar{x}$ denotes the class of $x \bmod \Gamma^{2}$.

We define a functor $\mathcal{I}_{P}(\quad)$ on $k$-algebras in the following way. For a $k$-algebra $R$ we set:

$$
\begin{gathered}
\mathcal{I}_{P}(R):=\left\{x_{i} \in \pi(V)(R), i=1, \ldots, k \mid r_{j}\left(x_{1}, \ldots, x_{k}\right)=0, \quad j=1, \ldots, l ;\right. \\
\left.\left\langle\bar{x}_{1}, \ldots, \bar{x}_{k}\right\rangle=\pi(V)(R)^{a b}\right\} .
\end{gathered}
$$

For each $n$ we define functors $\mathcal{I}_{P}^{n}(\quad)$. We set

$$
\mathcal{I}_{P}^{(n)}(R):=\left\{x_{i} \in \pi(V)(R) /_{\Gamma^{n+1} \pi(V)(R)}, i=1, \ldots, k \mid \ldots\right\}
$$

Proposition 5.1. The functor $\mathcal{I}_{P}^{n}(\quad)$ (resp. $\mathcal{I}_{P}(\quad)$ ) on $k$-algebras is representable by an affine, algebraic (resp. pro-algebraic) scheme over $k$, which we denote by $\mathcal{I}_{P}^{n}$ (resp. $\left.\mathcal{I}_{P}\right)$. The scheme $\mathcal{I}_{P}^{n}\left(\operatorname{resp} . \mathcal{I}_{P}\right)$ is an $\operatorname{Aut}\left(\pi(V) / \Gamma^{n+1} \pi(V)\right.$ )-torsor (resp. $\operatorname{Aut}(\pi(V))$-torsor) isomorphic to the $\operatorname{Aut}\left(\pi(V) / \Gamma^{n+1} \pi(V)\right)$-torsor (resp. Aut $(\pi(V))$-torsor) $\operatorname{Iso}\left(\pi_{0} / \Gamma^{n+1} \pi_{0} \times\right.$ $\left.\operatorname{Spec} k, \pi(V) / \Gamma^{n+1} \pi(V)\right)\left(\operatorname{resp} . I \operatorname{Iso}\left(\pi_{0} \times \operatorname{Spec} k, \pi(V)\right)\right)$. Hence we have $\mathcal{I}_{P}={\underset{\lim }{n}}_{\mathcal{I}_{P}^{n}}^{n}$.

Proof. Observe that any $f \in \mathcal{I}_{P}(R)$ determines a unique homomorphism $f^{\prime}: \pi_{1}(V(\mathbb{C}), x) \rightarrow$ $\pi(V)(R)$. By the universal property of the Malcev completion there exists a unique $R$ homomorphism (which is an isomorphism) $\bar{f}: \pi_{0}(R) \rightarrow \pi(V)(R)$ such that $r_{R} \circ \bar{f}=f^{\prime}$. Moreover any $R$-isomorphism $\varphi: \pi_{0}(R) \rightarrow \pi(V)(R)$ defines an element of $\mathcal{I}_{P}(R)$; the image of generators $A_{1} \ldots, A_{k}$ by $\varphi \circ r_{R}$. Hence we have a natural isomorphism $(*) \mathcal{I}_{P}(R) \approx$ $\operatorname{Iso}_{R}\left(\pi_{0}(R), \pi(V)(R)\right)$. But the functor $R \rightarrow \operatorname{Iso}_{R}\left(\pi_{0}(R), \pi(V)(R)\right)$ is representable by an affine, pro-algebraic scheme Iso $\left(\pi_{0} \times \operatorname{Spec} k, \pi(V)\right)$. Finally observe that the isomorphism $(*)$ of functors is compatible with action of $\operatorname{Aut}_{R}(\pi(V)(R))=(\operatorname{Aut}(\pi(V))(R))$.

For the given presentation $P=\left(\left(A_{i}\right)\right)$ of $\pi_{1}(V(\mathbb{C}), x)$, the sequence $\left(\theta_{x}^{-1}\left(A_{i}\right)\right)_{i} \in \mathcal{I}_{P}(\mathbb{C})$ and the sequence $\left(\left(\theta_{x}^{-1}\right)^{n}\left(A_{i}\right)\right)_{i} \in \mathcal{I}_{P}^{(n)}(\mathbb{C})$, where $\left(\theta_{x}^{-1}\right)^{n}: \pi_{1}(V(\mathbb{C}), x) \rightarrow \pi(V)(\mathbb{C}) / \Gamma^{n+1} \pi(V)(\mathbb{C})$ is induced by $\theta_{x}^{-1}$. We denote by $T_{r}\left(\left(\theta_{x}^{-1}\right)\left(A_{i}\right)\right)^{n}$ the smallest subtorsor of $\mathcal{I}_{P}^{(n)}$ defined over $k$, which contains $\left(\left(\theta_{x}^{-1}\right)^{n}\left(A_{i}\right)\right)_{i}$ as a $\mathbb{C}$-point. The corresponding subgroup of $\operatorname{Aut}\left(\pi(V) / \Gamma^{n+2} \pi(V)\right)$ we shall denote by $G_{r}\left(\left(\theta_{x}^{-1}\right)\left(A_{i}\right)\right)^{n}$.

The following result follows immediately from Proposition 5.1. 
Corollary 5.2. The isomorphism of $\operatorname{Aut}\left(\pi(V) / \Gamma^{n+1} \pi(V)\right)$-torsors from Proposition 5.1 identifies $G_{r}\left(\left(\theta_{x}^{-1}\right)\left(A_{i}\right)\right)^{n}$-torsor $T_{r}\left(\left(\theta_{x}^{-1}\right)\left(A_{i}\right)\right)^{n}$ with $G_{r}\left(\left(\theta_{x}^{-1}\right)_{0}\right)^{n}$-torsor $T_{r}\left(\left(\theta_{x}^{-1}\right)_{0}\right)^{n}$.

5.3. We recall from section 2 that we have an isomorphism

$$
\mathcal{C}_{x}: \pi_{1}^{B}(V(\mathbb{C}), x)(\mathbb{C}) \rightarrow \pi_{1}^{D R}(V, x)(\mathbb{C})
$$

and the associated $G_{r}\left(\mathcal{C}_{x}^{n}\right)$-torsor $T_{r}\left(\mathcal{C}_{x}^{n}\right)$. We shall relate this torsor to the torsors considered in this section.

Proposition 5.3. The group schemes $G_{r}\left(\left(\theta_{x}^{-1}\right)_{0}\right)^{n}$ and $G_{r}\left(\mathcal{C}_{x}^{n}\right)$ are isomorphic and the corresponding torsors $T_{r}\left(\left(\theta_{x}^{-1}\right)_{0}\right)^{n}$ and $T_{r}\left(\mathcal{C}_{x}^{n}\right)$ are isomorphic torsors.

Proof. It follows from 4.3, 4.4 and 4.5 that we have an isomorphism

$$
v: \pi(V) \rightarrow \pi_{1}^{D R}(V, x)
$$

induced by isomorphisms $P$ and $R$ of Hopf algebras. The homomorphism

$$
b: \pi_{1}(V(\mathbb{C}), x) \rightarrow \pi_{1}^{B}(V(\mathbb{C}), x)(\mathbb{Q})
$$

is given by evaluating iterated integrals on loops in $\pi_{1}(V(\mathbb{C}), x)$. There is a unique homomorphism

$$
b_{0}: \pi_{1}(V(\mathbb{C}), x)(\mathbb{Q}) \rightarrow \pi_{1}^{B}(V(\mathbb{C}), x)(\mathbb{Q})
$$

which is an isomorphism by the Sullivan theory of minimal models, such that $b_{0} \circ r_{\mathbb{Q}}=b$. We shall show that the diagram

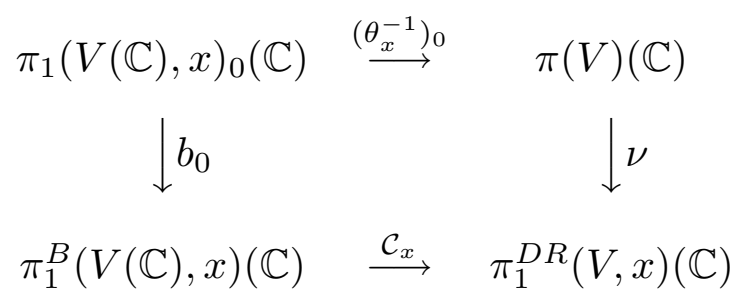

commutes. The morphism

$$
\pi_{1}(V(\mathbb{C}), x) \rightarrow \pi_{1}^{B} \pi_{1}(V(\mathbb{C}), x) \stackrel{\mathcal{C}_{x}}{\longrightarrow} \pi_{1}^{D R}(V, x)(\mathbb{C})
$$


is induced by evaluating iterated integrals on elements of $\pi_{1}(V(\mathbb{C}), x)$. Let exp $: \pi(V)(\mathbb{C}) \rightarrow$ $P(V)(\mathbb{C})$ be the exponential map. Then if follows from Proposition 3.9 and the formula $\int_{\alpha} \omega_{1}, \ldots, \omega_{n}=(-1)^{n} \int_{\alpha^{-1}} \omega_{n}, \ldots, \omega_{1}$ (see $\left.[\mathrm{Ch}]\right)$ that $\exp \left(\theta_{x}^{-1}(\alpha)\right)=1+\sum\left(\int_{\alpha} \omega_{i_{1}}, \ldots, \omega_{i_{k}}\right)$ $X_{i_{1}} \ldots, X_{i_{k}}$. The map $u^{*}: A l_{g}(P(V)) \rightarrow H_{D R}^{0}\left((V, x)^{\bullet}\right)$, which induces $u: \pi_{1}^{D R}(V, x) \stackrel{\nu^{-1}}{\longrightarrow} \pi(V)$ $\stackrel{\exp }{\longrightarrow} P(V)$, associates the coefficient at $X_{i_{1}} \ldots, X_{i_{k}}\left(X_{i}=\left(\omega_{i}\right)^{*}\right)$ to the class of $\left[\omega_{i_{1}} \otimes\right.$ $\left.\ldots, \otimes \omega_{i_{k}}\right]$ in $H_{D R}^{0}\left((V, x)^{\bullet}\right)$. This implies that the diagram commutes. The commutativity of the diagram implies the proposition.

\section{$\S$ 6. Partial calculations of $\mathcal{G}_{D R}\left(P^{1} \backslash\{0,1, \infty\}\right)$.}

6.1. Let $\mathbb{X}=\mathbb{P}_{\mathbb{Q}}^{1} \backslash\{0,1, \infty\}$. We recall that $\operatorname{Lie}(\mathbb{X})$ is a free Lie algebra over $\mathbb{Q}$ on $X=\left(\frac{d z}{z}\right)^{*}$ and $Y=\left(\frac{d z}{z-1}\right)^{*}$. We set $Z:=-X-Y$. Let $F_{k}(\operatorname{Lie}(\mathbb{X}))$ be a Lie subalgebra of Lie $(\mathbb{X})$ generated by commutators which contain $X$ at least $k$-times. We recall that $L(\mathbb{X})$ is a completion of $\operatorname{Lie}(\mathbb{X})$ with respect to the filtration induced by the lower central series. The closure of $F_{k}(\operatorname{Lie}(\mathbb{X}))$ in $L(\mathbb{X})$ we denote by $L_{k}(\mathbb{X})$ and the corresponding closed subgroup of $\pi(\mathbb{X})$ by $\pi_{k}$. Observe that $\left\{\pi_{k}\right\}_{k=0}^{\infty}$ is a filtration of $\pi(\mathbb{X})$ by normal subgroups. We set $\pi^{k}:=\pi(\mathbb{X}) / \pi_{k}$.

For any $\mathbb{Q}$-algebra $\mathbb{R}$, we set

$$
\begin{gathered}
\operatorname{Aut}^{*}(\pi(\mathbb{X}))(\mathbb{R}):=\left\{f \in \operatorname{Aut}\left(\pi(\mathbb{X})_{\mathbb{R}}\right) \mid \exists \alpha \in \mathbb{R}^{*}, \quad f(X)=\alpha X\right. \\
f(Y) \approx \alpha Y, \quad f(Z) \sim \alpha Z\} .
\end{gathered}
$$

Here $\approx$ means a conjugation by an element of $[\pi(\mathbb{X})(\mathbb{R}), \pi(\mathbb{X})(R)], \sim$ means a conjugation by an element of $\pi(\mathbb{X})(\mathbb{R})$.

Similarly we shall define

$$
\begin{array}{r}
\operatorname{Aut}^{*}\left(\pi^{k}\right)(\mathbb{R}):=\left\{f \in \operatorname{Aut}\left(\pi_{\mathbb{R}}^{k}\right) \mid \exists \alpha \in \mathbb{R}^{*}, \quad f(X)=\alpha X,\right. \\
f(Y) \approx \alpha Y, \quad f(Z) \sim \alpha Z\} .
\end{array}
$$

These functors on $\mathbb{Q}$-algebras are represented by affine, pro-algebraic group schemes over $\mathbb{Q}$, which we denote by $\operatorname{Aut}^{*}(\pi(\mathbb{X}))$ and $\operatorname{Aut}^{*}\left(\pi^{k}\right)$ respectively. 
6.1.2. The projection map $p_{k}: \pi(\mathbb{X}) \rightarrow \pi^{k}$ induces

$$
\left(p_{k}\right)_{*}: \operatorname{Aut}^{*}(\pi(\mathbb{X})) \longrightarrow \operatorname{Aut}^{*}\left(\pi^{k}\right)
$$

It follows from the fact that any $f \in \operatorname{Aut}^{*}(\pi(\mathbb{X}))$ maps $\pi_{k}$ into itself.

6.1.3. Observe also that the map

$$
\operatorname{Aut}^{*}(\pi(\mathbb{X})) \longrightarrow \operatorname{Out}(\pi(\mathbb{X})):=\operatorname{Aut}(\pi(\mathbb{X})) / \operatorname{Inn}(\pi(\mathbb{X}))
$$

is injective. This follows from the fact that

$$
\operatorname{Aut}^{*}(\pi(\mathbb{X})) \cap \operatorname{Inn}(\pi(\mathbb{X}))=\{I d\}
$$

6.1.4. We recall from section 10 that the monodromy homomorphism

$$
\theta:=\theta_{\overrightarrow{01}}: \pi_{1}(\mathbb{X}(\mathbb{C}), \overrightarrow{01}) \longrightarrow \pi(\mathbb{X})(\mathbb{C})
$$

is given by the formula

$$
\theta\left(S_{0}\right)=(-2 \pi i) X, \quad \theta\left(S_{1}\right)=\alpha^{-1} \cdot(-2 \pi i) Y \cdot \alpha, \quad \theta\left(S_{\infty}\right)=\alpha^{\prime-1} \cdot(-2 \pi i) Z \cdot \alpha^{\prime},
$$

where $\alpha=\alpha_{\overrightarrow{01}}^{\overrightarrow{10}}(X, Y) \in[\pi(\mathbb{X}) ; \pi(\mathbb{X})]$. Let us set $\Phi(\alpha):=(\theta(\alpha))^{-1}$. Let $\pi_{1}(k X) \subset$ $\pi_{1}(\mathbb{X}(\mathbb{C}) ; \overrightarrow{01})$ be a subgroup generated by commutators in $S_{0}$ and $S_{1}$ which contain $S_{0}$ at least $k$-times. Let $\pi_{1}^{k}:=\pi_{1}(\mathbb{X}(\mathbb{C}) ; \overrightarrow{01}) / \pi_{1}(k X)$. The monodromy homomorphism $\Phi$ induces

$$
\Phi(k): \pi_{1}^{k} \longrightarrow \pi^{k}(\mathbb{C})
$$

such that the induced map

$$
\Phi(k)_{0}:\left(\pi_{1}^{k}\right)_{0}(\mathbb{C}) \longrightarrow \pi^{k}(\mathbb{C})
$$

is an isomorphism. 
Proposition 6.1. We have

i) $G_{r}\left(\Phi_{0}\right) \subset \operatorname{Aut}^{*}(\pi(\mathbb{X}))$

ii) $G_{r}\left(\Phi(k)_{0}\right) \subset \operatorname{Aut}^{*}\left(\pi^{k}\right)$.

Proof. The presentation $P=\left\{S_{0}, S_{1}, S_{\infty} \mid S_{0} \cdot S_{1} \cdot S_{\infty}\right\}$ of $\pi_{1}(\mathbb{X}(\mathbb{C}), \overrightarrow{01})$ defines an $\operatorname{Aut}(\pi(\mathbb{X}))$-torsor $\mathcal{I}_{P}$ over Spec $\mathbb{Q}$ (see section 5$)$. The triple $\tau=\left((2 \pi i) X, \alpha^{-1} \cdot((2 \pi i) Y)\right.$. $\left.\alpha, \alpha^{\prime-1} \cdot((2 \pi i) Z) \cdot \alpha^{\prime}\right) \in \mathcal{I}_{P}(\mathbb{C})$. Let

$$
\begin{aligned}
T=\left\{\beta X, b^{-1} \cdot(\beta Y) \cdot b,\right. & b^{\prime-1} \cdot(\beta Z) \cdot b^{\prime} \mid \beta^{\prime} \in \mathbb{C}^{*}, \quad b \in[\pi(\mathbb{X}), \pi(\mathbb{X})], \\
& \left.b^{\prime} \in \pi(\mathbb{X}), \quad(\beta X) \cdot\left(b^{-1} \cdot(\beta Y) \cdot b\right) \cdot\left(b^{\prime-1} \cdot(\beta Z) \cdot b^{\prime}\right)=0\right\} .
\end{aligned}
$$

$T$ is an affine scheme over $\operatorname{Spec} \mathbb{Q}$ and $T$ is $\operatorname{Aut}^{*}(\pi(\mathbb{X}))$-torsor. Observe that $\tau \in T(\mathbb{C})$. Hence Corollary 5.2 implies that $G\left(\Phi_{0}\right) \subset \operatorname{Aut}^{*}(\pi(\mathbb{X}))$.

For each $k$, the presentation $P$ defines an $\operatorname{Aut}\left(\pi^{k}\right)$-torsor $\mathcal{I}_{P}^{k}$. For each $n$, the presentation $P$ defines an $\operatorname{Aut}\left(\pi^{k} / \Gamma^{n+2} \pi^{k}\right)$-torsor $\mathcal{I}_{P}^{k, n}$. Let $\tau_{k}$ (resp. $\tau_{k, n}$ ) be the image of $\tau$ in $\pi^{k}(\mathbb{C})\left(\right.$ resp. $\left.\pi^{k}(\mathbb{C}) / \Gamma^{n+2} \pi^{k}(\mathbb{C})\right)$. The smallest subtorsor of $\mathcal{I}_{P}^{k, n}$ defined over $\mathbb{Q}$, which contains $\tau_{k, n}$ as a complex point, we shall denote by $T\left(\tau_{k, n}\right)$. The corresponding group we shall denote by $G\left(\tau_{k, n}\right)$. By definition we have

$$
T\left(\tau_{k}\right):=\lim _{n} T\left(\tau_{k, n}\right), \quad G\left(\tau_{k}\right):={\underset{\lim }{n}}_{n} G\left(\tau_{k, n}\right)
$$

Let

$$
\begin{aligned}
T_{k}=\left\{\beta X, b^{-1} \cdot(\beta Y) \cdot b,\right. & b^{-1} \cdot(\beta Z) \cdot b^{\prime} \mid \beta \in \mathbb{C}^{*}, b \in\left[\pi^{k}(\mathbb{C}), \pi^{k}(\mathbb{C})\right], \\
b^{\prime} & \left.\in \pi^{k}(\mathbb{C}),(\beta X) \cdot\left(b^{-1} \cdot(\beta Y) \cdot b\right) \cdot\left(b^{\prime-1} \cdot(\beta Z) \cdot b^{\prime}\right)=0\right\} .
\end{aligned}
$$

$T_{k}$ is an affine scheme over $\mathbb{Q}$ and $T_{k}$ is an $\operatorname{Aut}^{*}\left(\pi^{k}\right)$-torsor. Observe that $\tau_{k} \in T_{k}(\mathbb{C})$. One must notice that analogues of Proposition 5.1 and Corollary 5.2 hold for the groups $\pi^{k}$ and $\pi_{1}^{k}$, the presentation $P$ and the isomorphism $\Phi(k)_{0}$. Then the analogue of Corollary 5.2 implies $G_{r}\left(\Phi(k)_{0}\right) \subset \operatorname{Aut}^{*}\left(\pi^{k}\right)$.

6.1.5. The projection $p_{k}: \pi(\mathbb{X}) \rightarrow \pi^{k}$ induces $\left(p_{k}\right)_{*}: \operatorname{Aut}^{*}(\pi(\mathbb{X})) \rightarrow \operatorname{Aut}^{*}\left(\pi^{k}\right)($ see 6.1 .2$)$ and $\left(p_{k}\right)_{*}^{\prime}: T \rightarrow T_{k}$. We have

$$
\left(p_{k}\right)_{*}\left(G_{r}\left(\Phi_{0}\right)\right)=G_{r}\left(\Phi(k)_{0}\right) \quad \text { and } \quad\left(p_{k}\right)_{*}^{\prime}(T(\tau))=T\left(\tau_{k}\right)
$$


The proof is analogous to the proof of Lemma 1.1.8.

6.1.6. Below we shall calculate the torsor $T\left(\tau_{2}\right)$ and the corresponding group $\mathrm{G}_{r}\left(\Phi(2)_{0}\right)$. By 6.1.5 we get some information about $\mathrm{G}_{r}\left(\Phi_{0}\right)$ and hence by 6.1 .3 about $\mathcal{G}_{D R}(\mathbb{X})$.

6.2. The elements $\theta\left(S_{0}\right)=(-2 \pi i) X$ and $\theta\left(S_{1}\right)=(-2 \pi i) Y+\sum_{k=2}^{\infty}(2 \pi i) \zeta(k)\left((Y X) Y^{k-1}\right)$ are images in $\pi^{2}(\mathbb{C})$ of the generators $S_{0}$ and $S_{1}$ by the monodromy homomorphism $\theta$ (see section 10). Let $\Theta_{n}\left(S_{0}\right)$ and $\Theta_{n}\left(S_{1}\right)$ be images of $\theta\left(S_{0}\right)^{-1}$ and $\theta\left(S_{1}\right)^{-1}$ in $\pi^{2}(\mathbb{C}) / \Gamma^{n+2} \pi^{2}(\mathbb{C})$. We shall calculate subtorsors $T_{n}:=T\left(\Theta_{n}\left(S_{0}\right), \Theta_{n}\left(S_{1}\right)\right)$ of $\mathcal{I}_{P}^{2, n}$ and the corresponding subgroups $G_{n}:=G\left(\Theta_{n}\left(S_{0}\right), \Theta_{n}\left(S_{1}\right)\right)$ of $\operatorname{Aut}\left(\pi^{2} / \Gamma^{n+2} \pi^{2}\right)$. Observe that by Lemma 1.1 .8 we have

$$
P_{n}^{n+1}\left(T_{n+1}\right)=T_{n} \quad \text { and } \quad P_{n}^{n+1}\left(G_{n+1}\right)=G_{n}
$$

where $P_{n}^{n+1}$ is induced by the projection $\pi^{2} / \Gamma^{n+3} \pi^{2} \rightarrow \pi^{2} / \Gamma^{n+2} \pi^{2}$.

0. Calculations of $T_{0}$ and $G_{0}$.

We have $\Theta_{0}\left(S_{0}\right)=(2 \pi i) X$ and $\Theta_{0}\left(S_{1}\right)=(2 \pi i) Y$. Observe that $(2 \pi i)$ is not a $k$-th root of a rational number for any $k=1,2,3 \ldots$ This implies that $T_{0}=\left\{\alpha X, \alpha Y \mid \alpha \in \mathbb{C}^{*}\right\}$ and $G_{0}=\left\{f_{\alpha}\left|f_{\alpha}(X)=\alpha X, f_{\alpha}(Y)=\alpha Y\right| \alpha \in \mathbb{C}^{*}\right\}$.

1. Calculations of $T_{1}$ and $G_{1}$.

We have $\Theta_{1}\left(S_{0}\right)=(2 \pi i) X$ and $\Theta_{1}\left(S_{1}\right)=(2 \pi i) Y$. The property $(*)$ implies that

$$
T_{1}=\left\{\alpha X, \alpha Y \mid \alpha \in \mathbb{C}^{*}\right\} \quad \text { and } \quad G_{1}=\left\{f_{\alpha}\left|f_{\alpha}(X)=\alpha X, f_{\alpha}(Y)=\alpha Y\right| \alpha \in \mathbb{C}^{*}\right\}
$$

2. Calculations of $T_{2}$ and $G_{2}$.

We have $\Theta_{2}\left(S_{0}\right)=(2 \pi i) X, \Theta_{2}\left(S_{1}\right)=(2 \pi i) Y+(-2 \pi i) \zeta(2)((Y X) Y)$. Observe that $(-2 \pi i) \zeta(2)=\frac{1}{24}(2 \pi i)^{3}$. This equality and the property $(*)$ (or the fact that $(-2 \pi i)$ is not a square root of a rational number) implies that

$$
\begin{aligned}
& T_{2}=\left\{\alpha X, \alpha Y+\frac{1}{24} \alpha^{3}((Y X) Y) \mid \alpha \in \mathbb{C}^{*}\right\} \text { and } \\
& G_{2}=\left\{f_{\alpha}\left|f_{\alpha}(X)=\alpha X, f_{\alpha}(Y)=\alpha Y\right| \alpha \in \mathbb{C}^{*}\right\}
\end{aligned}
$$

3. Calculations of $T_{3}$ and $G_{3}$. 
We have $\Theta_{3}\left(S_{0}\right)=(2 \pi i) X, \Theta_{3}\left(S_{1}\right)=(2 \pi i) Y+(-2 \pi i) \zeta(2)((Y X) Y)+$ $+(-2 \pi i) \zeta(3)\left((Y X) Y^{2}\right)$. Assume that $\operatorname{dim} G_{3}=\operatorname{dim} T_{3}=1$. Then it follows from $(*)$ and Corollary 14.2 that $G_{3}=G\left(0,0, c_{3}\right)$ where $c_{3} \in \mathbb{Q}$. As $T_{3}$ is a one-dimensional variety we have $T_{3}=\left\{\alpha X, \alpha Y+\frac{1}{24} \alpha^{3}((Y X) Y)+\beta_{3}\left((Y X) Y^{2}\right) \mid \alpha \in \mathbb{C}^{*}, \beta_{3} \in \mathbb{C}, p\left(\alpha, \beta_{3}\right)=0\right\}$ for some Laurent polynomial $p(x, y) \in \mathbb{Q}\left[x, \frac{1}{x}, y\right]$. Observe that to each value of $\alpha$ corresponds exactly one value $\beta_{3}(\alpha)$, because $T_{3}$ is a $G_{3}$-torsor. Hence $\beta_{3}(\alpha)=p(\alpha)$ where $p(x) \in$ $\mathbb{Q}\left[x, \frac{1}{x}\right]$. If $\left(\alpha X, \alpha Y+\frac{1}{24} \alpha^{3}((Y X) Y)+p(\alpha)(Y X) Y^{2}\right) \in T_{3}(\mathbb{C})$, then

$$
\left((\alpha t) X,(\alpha t) Y+\frac{1}{24}(\alpha t)^{3}((Y X) Y)+\left(p(\alpha) t^{4}+c_{3}\left(t-t^{4}\right) \alpha\right)\left((Y X) Y^{2}\right)\right) \in T_{3}(\mathbb{C}) .
$$

Hence $p(\alpha \cdot t)=p(\alpha) \cdot t^{4}+c_{3} \cdot\left(t-t^{4}\right) \cdot \alpha$. Therefore $p(x)=a \cdot x^{4}+c_{3} \cdot x$. The equality $(-2 \pi i) \zeta(3)=a(2 \pi i)^{4}+c_{3}(2 \pi i)$ implies $a=0$ and $c_{3}=-\zeta(3)$. Therefore we get

$$
\operatorname{dim} G_{3}=1 \quad \text { if and only if } \quad \zeta(3) \in \mathbb{Q} .
$$

n. Calculations of $T_{n}$ and $G_{n}$.

We have $\Theta_{n}\left(S_{0}\right)=(2 \pi i) X, \Theta_{n}\left(S_{1}\right)=(2 \pi i) Y+\sum_{k=2}^{n-1}(-2 \pi i) \zeta(k)\left((Y X) Y^{k-1}\right)+$ $+(-2 \pi i) \zeta(n)\left((Y X) Y^{n-1}\right)$. Assume that $G_{n-1}=G\left(0,0, c_{3}, 0, c_{5}, 0, c_{7}, \ldots \mid 0,0, \varepsilon_{3}, 0, \varepsilon_{5}, 0\right.$, $\left.\varepsilon_{7}, \ldots\right)\left(\right.$ see Corollary 14.4), where $c_{1}=c_{2}=c_{4}=\cdots=c_{2 k}=\cdots=0, c_{2 k+1}=-\zeta(2 k+1)$ and $\varepsilon_{2 k+1}=0$ if $\zeta(2 k+1) \in \mathbb{Q}$, and $c_{2 k+1}=0$ and $\varepsilon_{2 k+1}=1$ if $\zeta(2 k+1) \notin \mathbb{Q}$.

Then we have that the torsor $T_{n-1}$ corresponding to the group $G_{n-1}$ is given by

$$
\begin{aligned}
& T_{n-1}=\left\{\alpha X, \alpha Y+\sum_{k=2, k \text {-pair }}^{n-1} r_{k} \cdot \alpha^{k+1}\left((Y X) Y^{k-1}\right)+\sum_{k=3, k \text {-impair }}^{n-1}\left(\left(1-\varepsilon_{k}\right) c_{k} \cdot \alpha+\right.\right. \\
& \left.\left.+\varepsilon_{k} \beta_{k}\right)\left((Y X) Y^{k-1}\right) \mid \alpha \in \mathbb{C}^{*}, \forall k \beta_{k} \in \mathbb{C}\right\} .
\end{aligned}
$$

Assume that $n=2 p$. Then $\zeta(n)(-2 \pi i)=r_{n} \cdot(2 \pi i)^{n+1}, r_{n} \in \mathbb{Q}$. This equality, the fact that $(2 \pi i)^{n} \notin \mathbb{Q}$ and the property $(*)$ imply that

$$
G_{n}=G\left(0,0, c_{3}, 0, c_{5}, \ldots, c_{n-1}, 0 \mid 0,0, \varepsilon_{3}, 0, \ldots, \varepsilon_{n-1}, 0\right)
$$

and 


$$
\begin{aligned}
& T_{n}=\left\{\alpha X, \alpha Y+\sum_{k=2, k \text {-pair }}^{n} r_{k} \cdot \alpha^{k+1}\left((Y X) Y^{k-1}\right)+\sum_{k=3, k \text {-impair }}^{n}\left(\left(1-\varepsilon_{k}\right) c_{k} \cdot \alpha+\right.\right. \\
& \left.\left.+\varepsilon_{k} \cdot \beta_{k}\right)\left((Y X) Y^{k-1}\right) \mid \alpha \in \mathbb{C}^{*}, \underset{k}{\forall}, \beta_{k} \in \mathbb{C}\right\} .
\end{aligned}
$$

Assume that $n=2 p+1$. Assume that $\operatorname{dim} G_{n}=\operatorname{dim} G_{n-1}$. Then

$$
G_{n}=G\left(0,0, c_{3}, \ldots, 0, c_{n} \mid 0,0, \varepsilon_{3}, 0, \varepsilon_{5}, \ldots, 0,0\right) \quad(\text { by Corollary 14.4) }
$$

and

$$
\begin{aligned}
& T_{n}=\left\{\alpha X, \alpha Y+\sum_{k=2, k \text {-pair }}^{n-1} r_{k} \alpha^{k+1}\left((Y X) Y^{k-1}\right)+\sum_{k=3, k \text {-impair }}^{n-1}\left(\left(1-\varepsilon_{k}\right) \cdot c_{k} \cdot \alpha+\right.\right. \\
& \left.\left.+\varepsilon_{k} \cdot \beta_{k}\right)\left((Y X) Y^{k-1}\right)+\beta_{n}\left(Y^{n} X\right) \mid \alpha \in \mathbb{C}^{*} ; \forall k, \beta_{k} \in \mathbb{C} ; p\left(\alpha, \varepsilon_{3} \cdot \beta_{3}, \varepsilon_{5} \cdot \beta_{5}, \ldots, \beta_{n}\right)=0\right\}
\end{aligned}
$$

for some polynomial $p\left(x, y_{3}, y_{5}, \ldots, y_{n}\right) \in \mathbb{Q}\left[x, \frac{1}{x}, y_{3}, y_{5}, \ldots, y_{n}\right]$.

Assume that for some $\alpha_{0}$ there are two different $\beta_{n}$. Then there is $g$ in $G_{n}$ such that $g(X)=X, g(Y)=Y+\cdots+b_{n}\left((Y X) Y^{n-1}\right)$ with $b_{n} \neq 0$. Then it follows from the proof of Lemma 14.5 that $G_{n}=G\left(0,0, c_{3}, 0, \ldots, 0,0 \mid 0,0, \varepsilon_{3}, 0, \ldots, 0,1\right)$ and $\operatorname{dim} G_{n}=$ $\operatorname{dim} G_{n-1}+1$. Hence for any $\alpha \in \mathbb{C}^{*}$ there is a unique $\beta_{n}$ corresponding to that $\alpha$. Observe that $\beta_{n}$ is an algebraic function of $\alpha, \beta_{3}, \beta_{5}, \ldots$, which depends only on $\alpha$. Hence $\beta_{n}=p(\alpha)$ for some $p(x) \in \mathbb{Q}\left[x, \frac{1}{x}\right]$.

Choose any $f \in G_{n}$. Then $f(X)=t X, f(Y)=t Y+\sum_{k=3, k \text {-impair }}^{n-1}\left(\left(1-\varepsilon_{k}\right) \cdot c_{k} \cdot\left(t-t^{k+1}\right)+\right.$ $\left.\varepsilon_{k} \cdot b_{k}\right)\left((Y X) Y^{k-1}\right)+c_{n} \cdot\left(t-t^{n+1}\right)\left((Y X) Y^{n-1}\right)$ for some $t \in \mathbb{C}^{*}, b_{3}, b_{5}, \ldots, b_{n-2} \in \mathbb{C}$. Acting by $f$ on a chosen element of $T_{n}$ we get

$$
\begin{aligned}
& \left((\alpha \cdot t) X,(\alpha \cdot t) Y+\sum_{k=2, k \text {-pair }}^{n-1} r_{k}(\alpha \cdot t)^{k+1}\left((Y X) Y^{k-1}\right)+\right. \\
& +\sum_{k=3, k \text {-impair }}^{n-1}\left(\alpha \cdot\left(1-\varepsilon_{k}\right) \cdot c_{k} \cdot\left(t-t^{k+1}\right)+\alpha \cdot \varepsilon_{k} \cdot b_{k}+\left(1-\varepsilon_{k}\right) \cdot c_{k} \cdot \alpha \cdot t^{k+1}+\right. \\
& \left.+\varepsilon_{k} \cdot \beta_{k} \cdot t^{k+1}\right)\left((Y X) Y^{k-1}\right)+\left(\alpha \cdot c_{n} \cdot\left(t-t^{n+1}\right)+\beta_{n} \cdot t^{n+1}\right)\left((Y X) Y^{n-1}\right) .
\end{aligned}
$$

Therefore we get

$$
p(\alpha \cdot t)=c_{n} \cdot \alpha \cdot\left(t-t^{n+1}\right)+p(\alpha) \cdot t^{n+1} .
$$


This implies $p(x)=a \cdot x^{n+1}+c_{n} \cdot x$. The equality $\zeta(n)(-2 \pi i)=a \cdot(2 \pi i)^{n+1}+c_{n} \cdot(2 \pi i)$ implies $p(x)=c_{n} \cdot x$ and $c_{n}=-\zeta(n)$. Therefore we get

$$
\operatorname{dim} G_{n}=\operatorname{dim} G_{n-1} \quad \text { if and only if } \quad \zeta(n) \in \mathbb{Q} .
$$

The final result is the following.

Proposition 6.2. Let $G=\overleftarrow{n}_{n}^{\lim } G_{n}$. Then

$$
G=G\left(0,0, c_{3}, 0, c_{5}, 0, c_{7}, \ldots, 0, c_{2 k+1}, 0 \ldots \mid 0,0, \varepsilon_{3}, 0, \varepsilon_{5}, 0 \ldots, 0, \varepsilon_{2 k+1}, 0 \ldots\right)
$$

where $\varepsilon_{2 k+1}=0$ if and only if $\zeta(2 k+1) \in \mathbb{Q}$, and then $c_{2 k+1}=\zeta(2 k+1)$.

Corollary 6.3. The group $G$ contains the group $H:=\left\{f_{\alpha}\left|f_{\alpha}(X)=\alpha X, f_{\alpha}(Y)=\alpha Y\right|\right.$ $\left.\alpha \in \mathbb{C}^{*}\right\}$ if and only if all numbers $\zeta(2 k+1)$ are irrational.

Proof. It follows from Proposition 6.2 that all $\zeta(2 k+1)$ are irrational if and only if $G=$ $G(0,0,0, \ldots, 0, \ldots \mid 0,0,1,0,1,0, \ldots 0,1,0,1,0, \ldots)\left(\right.$ all $c_{i}=0, \varepsilon_{1}=0$, all $\varepsilon_{2 k}=0$ and all $\varepsilon_{2 k+1}=1$ for $\left.k=1, \ldots\right)$, but then $H \subset G$. Let $H \subset G=G\left(0,0, c_{3}, 0, c_{5}, \ldots, 0, c_{2 k+1}, 0 \ldots \mid\right.$ $\left.0,0, \varepsilon_{3}, 0, \varepsilon_{5}, 0, \ldots, 0, \varepsilon_{2 k+1}, 0, \ldots\right)$. Then there is $f \in G$ such that $f(X)=\alpha X, f(Y)=$ $\alpha Y+\sum_{k=1}^{\infty} x_{2 k+1}\left((Y X) Y^{2 k}\right)$, where all $x_{2 k+1} \neq 0$. Corollary 14.5 implies that $G=G(0,0 \ldots, 0, \ldots$ $0,0,1,0,1, \ldots 0,1,0, \ldots)\left(\right.$ all $c_{i}=0, \varepsilon_{1}=\varepsilon_{2 k}=0, \varepsilon_{2 k+1}=1$ for $\left.k=1,2, \ldots\right)$.

Corollary 6.4. Let $\mathcal{G}$ be the smallest subgroup of $\operatorname{Aut}\left(\pi^{2}\right)$ defined over $\mathbb{Q}$, which contains $G$ and $H$. Then $\mathcal{G}=G(0,0 \ldots, 0, \ldots \mid 0,0,1,0,1,0 \ldots)\left(\right.$ all $c_{i}=0, \varepsilon_{1}=\varepsilon_{2 k}=0, \varepsilon_{2 k+1}=1$ for $k=1,2, \ldots)$.

Proof. The group $\mathcal{G}$ contains $f$ such that $f(X)=X, f(Y)=Y+\sum_{k=1}^{\infty} x_{2 k+1}\left((Y X) Y^{2 k}\right)$ where all $x_{2 k+1} \neq 0$. Lemma 14.5 implies that the corollary.

Corollary 6.5. Image of $\mathcal{G}_{D R}(\mathbb{X})$ in $\operatorname{Aut}\left(\pi^{2}\right)$ is the group $G$ from Proposition 6.2.

6.6. We recall from section 10 that the monodromy homomorphism

$$
\theta:=\theta_{\overrightarrow{01}}: \pi_{1}(\mathbb{X}, \overrightarrow{01}) \longrightarrow \pi_{2}(\mathbb{X})
$$


is given by

$$
S_{0} \rightarrow(-2 \pi i) X, \quad S_{1} \rightarrow(-2 \pi i) Y+\sum_{i=0, j=0}^{\infty}(2 \pi i) \alpha_{i+1, j+1}\left((Y X) X^{i} Y^{j+1}\right)
$$

(see section 10, formula (7)). We shall describe a torsor and a corresponding group associated to $\theta$. It is an observation of Drinfeld that the numbers $\alpha_{i, j}$ satisfy the equation

$$
-1+\sum_{n \geq 0, m \geq 1} \alpha_{n+1, m} \cdot u^{n+1} \cdot v^{m}=-\exp \left(-\sum_{k=2}^{\infty} \sum_{\substack{i+j=k \\ i \geq 1, j \geq 1}} \frac{(k-1) !}{i ! j !} \cdot \alpha_{1, k-1} \cdot u^{i} \cdot v^{j}\right)
$$

(see [Dr]). We have also $\alpha_{1, k-1}=\alpha_{k-1,1}=\zeta(k)$. Now we can describe the monodromy homomorphism $\theta$ in the following way

$$
S_{0} \rightarrow(-2 \pi i) X, \quad S_{1} \rightarrow\left((-2 \pi i) Y, \exp \left(-\sum_{k=2}^{\infty} \sum_{\substack{i+j=k \\ i \geq 1, j \geq 1}} \frac{(k-1) !}{i ! j !} \cdot \alpha_{1, k-1} \cdot X^{i} \cdot Y^{j}\right)\right)
$$

(see section 14 for the notation $(\ldots, \exp (\ldots))$ ). Repeating arguments from the proof of 6.2-6.4 and using Propositions 14.1 and 14.3 instead of Corollaries 14.2 and 14.4 we get the following result.

Theorem 6.7. i) The torsor $T(\theta)$ associated to $\theta$ (i.e. to the map obtained from $\theta$ by taking $\theta^{-1}$, passing to suitable quotient and $\mathbb{C}$-completion) is equal

$$
\begin{aligned}
& \left\{\alpha X,\left(\alpha Y, \exp \left(\sum_{k=1}^{\infty} \sum_{\substack{i+j=2 k \\
i \geq 1, j \geq 1}} \frac{(k-1) !}{i ! j !} r_{2 k} \alpha^{2 k}+\right.\right.\right. \\
& \left.\left.\sum_{\substack{k=3 \\
k \text { odd }}}^{\infty} \sum_{\substack{i \geq j=k \\
i \geq 1, j \geq 1}} \frac{(k-1) !}{i ! j !}\left((1-\varepsilon(k)) \cdot c_{k} \cdot\left(1-\alpha^{k}\right)+\varepsilon(k) \cdot b_{1, k-1}\right) X^{i} Y^{j}\right)\right) \mid \\
& \alpha \in \mathbb{C}^{*}, \quad-\zeta(2 k)=r_{2 k} \cdot(2 \pi i)^{2 k}, \underset{\substack{k \\
k}}{\forall} b_{1, k-1} \in \mathbb{C}, \quad c_{k}=\zeta(k) \\
& \text { and } \varepsilon(k)=0 \quad \text { if } \zeta(k) \in \mathbb{Q}, \quad \varepsilon(k)=1 \quad \text { if } \quad \zeta(k) \notin \mathbb{Q}\} .
\end{aligned}
$$

ii) The corresponding group

$$
\begin{aligned}
& G(\theta)=\left\{f \in \operatorname{Aut}^{*}\left(\pi_{2}(\mathbb{X})\right) \mid f(X)=t X, f(Y)=\right. \\
& \left(t Y, \exp \left(\sum_{\substack{k=3 \\
k \text {-odd }}} \sum_{\substack{i \geq j=k \\
i \geq 1, j \geq 1}} \frac{(k-1) !}{i ! j !}(1-\varepsilon(k)) \cdot c_{k} \cdot\left(1-t^{k}\right)+\varepsilon(k) \beta_{1, k-1}\right) X^{i} Y^{j}\right) \mid \\
& \left.t \in \mathbb{C}^{*}, \beta_{1, k-1} \in \mathbb{C}\right\} .
\end{aligned}
$$


iii) Let $G \subset \operatorname{Aut}^{*}\left(\pi_{2}(\mathbb{X})\right)$ be the smallest group which contains $G(\theta)$ and $G_{0}:=\left\{f_{t} \in\right.$ $\left.\operatorname{Aut}^{*}\left(\pi_{2}(\mathbb{X})\right)\left|f_{t}(X)=t X, f_{t}(Y)=t Y\right| t \in \mathbb{C}^{*}\right\}$. Then

$$
\begin{aligned}
& G=\left\{f \in \operatorname{Aut}^{*}\left(\pi_{2}(\mathbb{X})\right) \mid f(X)=t X, f(Y)=\right. \\
& \left.\left(t Y, \exp \left(\sum_{\substack{k=3 \\
k \text { odd }}}^{\infty} \sum_{\substack{i+j=k \\
i \geq 1, j \geq 1}} \frac{(k-1) !}{i ! j !} \beta_{1, k-1} X^{i} Y^{j}\right)\right) \mid t \in \mathbb{C}^{*}, \beta_{1, k-1} \in \mathbb{C}\right\} .
\end{aligned}
$$

\section{$\S$ 7. Homotopy relative tangential base points on $P^{1}(\mathbb{C}) \backslash\left\{a_{1}, \cdots, a_{n+1}\right\}$.}

7.1. Let $X=P^{1}(\mathbb{C}) \backslash\left\{a_{1}, \cdots, a_{n+1}\right\}$. Let $T_{x}\left(P^{1}(\mathbb{C})\right)$ be the tangent space to $P^{1}(\mathbb{C})$ in $x$. Let us set $\hat{X}=X \cup \bigcup_{i=1}^{n+1}\left(T_{a_{i}}\left(P^{1}(\mathbb{C})\right) \backslash\{0\}\right)$.

Let $J^{\prime}(\hat{X})$ be the set of all continous maps from the closed unit interval $[0 ; 1]$ to $P^{1}(\mathbb{C})$ such that

i) $\varphi((0,1)) \subset X$;

ii) if $\varphi(0)=a_{i}$ then $\varphi$ is smooth near $a_{i}$ and $\dot{\varphi}(0) \neq 0$, and if $\varphi(1)=a_{k}$ then $\varphi$ is smooth near $a_{k}$ and $\dot{\varphi}(1) \neq 0$.

In the sequel we shall identify $\dot{\varphi}(0)$ (resp. $\dot{\varphi}(1)$ ) with a tangent vector to $\varphi$ in $T_{a_{i}}\left(P^{1}(\mathbb{C})\right)\left(\right.$ resp. $T_{a_{k}}\left(P^{1}(\mathbb{C})\right)$. This tangent vector we shall denote also by $\dot{\varphi}(0) \in T_{a_{i}}\left(P^{1}(\mathbb{C})\right)$ (resp. $\dot{\varphi}(1) \in T_{a_{k}}\left(P^{1}(\mathbb{C})\right)$.

If $\varphi(0)=x \in X$ and $\varphi(1)=y \in X$ then we say that $\varphi$ is a path from $x$ to $y$.

If $\varphi(0)=a_{i}\left(\right.$ resp. $\left.\varphi(1)=a_{k}\right)$ then we say that $\varphi$ is a path from $\dot{\varphi}(0) \in T_{a_{i}}\left(P^{1}(\mathbb{C})\right)$ (resp. to $-\dot{\varphi}(1) \in T_{a_{k}}\left(P^{1}(\mathbb{C})\right)$ and we shall write $\varphi(0)=\dot{\varphi}(0)(\operatorname{resp} . \varphi(1)=-\dot{\varphi}(1))$.

To $J^{\prime}(\hat{X})$ we joint all constant maps from $[0,1]$ to $\hat{X}$ and the resulting set we denote by $J(\hat{X})$.

We shall define a relation of homotopy in the set $J(\hat{X})$. Let $\varphi, \psi \in J(\hat{X})$. If $\varphi(0)=$ $\psi(0)=x \in X$ and $\varphi(1)=\psi(1)=y \in X$ then we say that $\varphi$ and $\psi$ are homotopic if they are homotopic maps in the $\operatorname{space} \operatorname{map}([0,1], 0,1 ; X, x, y)$.

If $\varphi(0)=\psi(0)=v \in T_{a_{i}}\left(P^{1}(\mathbb{C})\right)$ and $\varphi(1)=\psi(1)=y \in X$ then we say that $\varphi$ and $\psi$ are homotopic if there is a homotopy

$H_{s} \in \operatorname{map}\left([0,1], 0,1 ; X \cup\left\{a_{i}\right\}, a_{i}, y\right)$ such that

i) $H_{s} \in J(\hat{X})$ and $H_{s}(0)=v$ for all $s \in[0,1]$; 
ii) $H_{s}((0,1)) \subset X$ for all $s \in[0,1]$;

iii) $H_{0}=\varphi$ and $H_{1}=\psi$.

We left to the reader the cases when $\varphi(0)=\psi(0)=x \in X, \varphi(1)=\psi(1)=w \in$ $T_{a_{k}}\left(P^{1}(\mathbb{C})\right)$ and $\varphi(0)=\psi(0)=v \in T_{a_{i}}\left(P^{1}(\mathbb{C})\right), \varphi(1)=\psi(1)=w \in T_{a_{k}}\left(P^{1}(\mathbb{C})\right)$.

Let $\varphi \in J(\hat{X})$ be such that $\varphi(0)=\varphi(1)=v \in T_{a_{i}}\left(P^{1}(\mathbb{C})\right)$ and let $\psi \in J(\hat{X})$ be a constant map equal to $v$. We say that $\varphi$ and $\psi$ are homotopic if there is a homotopy

$$
H_{s} \in \operatorname{map}\left([0,1], 0,1 ; X \cup\left\{a_{i}\right\}, a_{i}, a_{i}\right)
$$

such that

i) $H_{s} \in J(\hat{X})$ and $H_{s}(0)=H_{s}(1)=v$ for all $s \in[0,1]$;

ii) $H_{s}((0,1)) \subset X$ for all $s \in[0,1]$;

iii) $H_{0}=\varphi$ and $H_{1}(t)=a_{i}$ for $t \in[0,1]$.

Observe that $G_{t}:=H_{1-t}$ defines a homotopy between $\psi$ and $\varphi$.

With the definition given above paths $\alpha, \beta \in J(\widehat{C \backslash\{0\}})$ are not homotopic.

We shall write $\varphi \sim \psi$ if $\varphi$ and $\psi$ are homotopic. The relation $\sim$ is an equivalence relation on the set $J(\hat{X})$. Let $\iota(\hat{X}):=J(\hat{X}) / \sim$ be the set of equivalence classes.

We define a partial composition in $\iota(\hat{X})$ in the following way. Let $\phi, \Psi \in \iota(\hat{X})$ and let $\varphi, \psi \in J(\hat{X})$ be its representatives.

If $\varphi(1)=\psi(0)=y \in X$ then we set $\Psi \circ \phi:=[\psi \circ \varphi]$, the class of $\psi \circ \varphi$ in $\pi(\hat{X})$.

If $\varphi(1)=\psi(0)=v \in T_{a_{i}} P^{1}(\mathbb{C})$ then we can assume that $\varphi$ and $\psi$ coinside near $a_{i}$ and we define $\Psi \circ \phi:=\left[\psi^{\varepsilon} \cdot \varphi_{\eta}\right]$, where $\varphi_{1-\varepsilon}:=\varphi_{[0,1-\varepsilon]}, \psi_{\eta}:=\psi_{[[\eta, 1]}$ and $\varphi(1-\varepsilon)=\psi(\eta)$.

The map pr: $J(\hat{X}) \rightarrow \hat{X} \times \hat{X} / \operatorname{pr}(\varphi)=(\varphi(0), \varphi(1))$ which associates to a path its beginning $(\varphi(0))$ and its end $(\varphi(1))$ agrees with the relation $\sim$ and it defines $p: \iota(\hat{X}) \rightarrow$ $\hat{X} \times \hat{X}$. The partial composition $o$ makes $p: \iota(\hat{X}) \rightarrow \hat{X} \times \hat{X}$ into a groupoid over $\hat{X} \times \hat{X}$. 
Let $x \in \hat{X}$. We set $\pi_{1}(X, x):=p^{-1}(x, x)$. This is a fundamental group with a base point in $x \in \hat{X}$.

7.2. We shall construct a family of horizontal sections of $\omega_{x}$, where a base point $x$ is replace by a tangent vector.

Let us set $V=\mathbb{C} \backslash\left\{a_{1}, \cdots, a_{n}\right\}$. Let $x_{0} \in \mathbb{C}$ and let $\delta:[0,1] \ni t \rightarrow a_{i}+t .\left(x_{0}-a_{i}\right)$ be an interval joining $a_{i}$ and $x_{0}$. Let $\gamma$ be a path from $a_{i}$ to $z \in V$ (not passing through any $\left.a_{k}, k=1, \cdots n\right)$ tangent to $\delta$ in $a_{i}$. We assume that in a small neighbourhood of $a_{i}$ the path $\gamma$ coincides with $\delta$.

Observe that $v=x_{0}-a_{i}$ can be canonically identified with a tangent vector to $\mathbb{C}$ in $a_{i}$. Let $\omega_{1}=\frac{d z}{z-a_{1}}, \cdots, \omega_{n}=\frac{d z}{z-a_{n}}$. We set

$$
\Lambda_{a_{i}, v}\left(\alpha_{1}, \cdots, \alpha_{k}\right)(z):=\int_{a_{i}, \gamma}^{z} \omega_{\alpha_{1}}, \cdots, \omega_{\alpha_{k}} \quad \text { if } \quad \alpha_{1} \neq i
$$

Let $\varepsilon \in i m(\delta)$ be near $a_{i}$. Let $\gamma_{\varepsilon}$ be a part of $\gamma$ from $\varepsilon$ to $z$, and let $\delta_{\varepsilon}$ be a part of $\delta$ from $\varepsilon$ to $x_{0}$. We set

$$
\begin{aligned}
& \Lambda_{a_{i}, v}\left(i, \cdots, i, \alpha_{k+1} \cdots, \alpha_{l}\right)(z):= \\
& \lim _{\varepsilon \rightarrow a_{i}} \int_{\varepsilon, \gamma_{\varepsilon}}^{z}\left(\int_{x_{0}, \gamma_{\varepsilon}+\left(\delta_{\varepsilon}\right)^{-1}}^{z} \frac{d z}{x-a_{i}}, \cdots, \frac{d z}{z-a_{i}}\right) \omega_{\alpha_{k+1}}, \cdots, \omega_{\alpha_{l}}
\end{aligned}
$$

if $\alpha_{k+1} \neq i$,

and

$$
\Lambda_{a_{i}, v}(i, \cdots, i)(z)=\int_{x_{0}, \gamma_{\varepsilon}+\delta_{\varepsilon}^{-1}}^{z} \frac{d z}{z-a_{i}}, \cdots, \frac{d z}{z-a_{i}} .
$$

Lemma 7.2.1. The integrals $\Lambda_{a_{i}, v}\left(\alpha_{1}, \cdots, \alpha_{k}\right)(z)$ exist and they are analytic, multivalued functions on $V$.

Proof. Assume that $\alpha_{t} \neq i$ for $t \leq l$ and $\alpha_{l+1}=i$. The function $g(z):=\int_{a_{i}}^{z} \omega_{\alpha_{1}}, \cdots, \omega_{\alpha_{l}}$ is analytic, multivalued on $V \cup\left\{a_{i}\right\}$ and vanishes in $a_{i}$. Hence the integral $g_{1}(z):=$ $\int_{a_{i}}^{z} g(z) \frac{d z}{z-a_{i}}$ exists, the function $g_{1}(z)$ is analytic, multivalued on $V \cup\left\{a_{i}\right\}$ and vanishes in $a_{i}$. Hence by induction we get that $\Lambda_{a_{i}, v}\left(\alpha_{1}, \cdots, \alpha_{n}\right)(z)$ exists, and it is analytic, multivalued on $V \cup\left\{a_{i}\right\}$. 
Assume now that $\alpha_{t}=i$ for $t \leq l$ and $\alpha_{l+1} \neq i$. Without loss of generality we can assume that $a_{i}=0$ and $x_{0}=1$.

Observe that $\lim _{\varepsilon \rightarrow 0} \int_{x_{0}, \gamma_{\varepsilon} \circ\left(\delta_{\varepsilon}\right)^{-1}}^{z} z^{n}(\log z)^{m} d z=z^{n+1}\left(\sum_{i=0}^{m} \beta_{i}(\log z)^{m-i}\right)$ where $\beta_{i}$ are rational numbers. The function $z^{g}(\log z)^{p}$ for $g$ and $p$ positive integers, is analytic, multvalued on $V$, continous on any small cone with a vertex in $a_{i}$ (0 in this case) and it vanishes in $a_{i}$. The function $\frac{1}{z-a_{j}}$ for $j \neq i$ is bounded on any sufficiently small neighbourhood of $a_{i}$. Hence the integral

$$
\lim _{\varepsilon \rightarrow a_{i}} \int_{\varepsilon, \gamma_{\varepsilon}}^{z}\left(\int_{x_{0}, \gamma_{\varepsilon} \circ\left(\delta_{\varepsilon}\right)^{-1}} \frac{d z}{z-a_{i}}, \cdots, \frac{d z}{z-a_{i}}\right) \frac{d z}{z-a_{j}}, \cdots, \frac{d z}{z-a_{i_{k}}}
$$

is an analytic, multivalued function on $V$, continous, univalued on any small cone with a vertex in $a_{i}$ and it vanishes in $a_{i}$.

Let us set

$$
\Lambda_{V}(z ; v, \gamma)=1+\sum(-1)^{k} \Lambda_{a_{i}, v}\left(\alpha_{1}, \cdots, \alpha_{k}\right)(z) X_{\alpha_{k}} \cdots X_{\alpha_{1}}
$$

We recall that $X_{1}, \cdots, X_{n}$ are duals of $\frac{d z}{z-a_{1}}, \cdots, \frac{d z}{z-a_{n}}$.

Lemma 7.2.2. The map

$$
V \ni z \rightarrow\left(z, \Lambda_{V}(z ; v, \gamma)\right) \in V \times P(V)
$$

is horizontal with respect to $\omega_{V}$.

It rests to define functions $\Lambda_{X}(z ; v, \gamma)$ if $a_{i}=\infty$ or all $a_{i}$ are different from $\infty$.

Let $f: Y=\mathbb{C} \backslash\left\{b_{1}, \cdots, b_{n}\right\} \rightarrow X=P^{1}(\mathbb{C}) \backslash\left\{a_{1}, \cdots, a_{n+1}\right\}$ be a regular map of the form $\frac{a z+b}{c z+d}$ with $\operatorname{det}\left(\begin{array}{ll}a & b \\ c & d\end{array}\right) \neq 0$. It follows from corollary 3.8 that $f_{*}\left(\Lambda_{Y}(z ; y, \gamma)\right)=$ $\Lambda_{X}(f(x) ; f(y), f(\gamma))$ if $y \in Y$. We shall use this fact to define $\Lambda_{X}(z ; v, \gamma)$ where $v$ is a tangent vector to $P^{1}(\mathbb{C})$ in $a_{i}$ and $\gamma$ is a path from $a_{i}$ to $z$, which is tangent to $v$ and $f^{-1}(\gamma)$ coincides with $f^{-1}(v)$ near $f^{-1}\left(a_{i}\right)$.

We set

$$
\Lambda_{X}(z ; v, \gamma):=f_{*}\left(\Lambda_{Y}\left(f^{-1}(z) ; f_{*}^{-1}(v), f^{-1}(\gamma)\right)\right)
$$

It is clear that $\Lambda_{X}(z ; v, \gamma)$ does not depend on the choice of $f$. 
Lemma 7.2.3. The map

$$
X \ni z \rightarrow\left(z, \Lambda_{X}(z ; v, \gamma)\right) \in X \times P(X)
$$

is horizontal with respect to $\omega_{X}$.

We set $L_{X}(z ; v, \gamma):=\log \Lambda_{X}(z ; v, \gamma)$. If we are dealing with only one space $X$ we shall usually omit subscript $X$ and we shall write $\Lambda(z ; v, \gamma)$ and $L(z ; v, \gamma)$, or even $\Lambda_{v}(z ; \gamma)$ and $L_{v}(z ; \gamma)$, or $\Lambda_{v}(z)$ and $L_{v}(z)$.

We summarize the constractions from 7.1 and 7.2 in the following proposition.

Proposition 7.2.4. The functions $\Lambda_{X}(z ; v, \gamma)$ and $L_{X}(z ; v, \gamma)$ depend only on the homotopy class of $\gamma$ in $\iota(\hat{X})=J(\hat{X}) / \sim$.

$\S$ 8. Generators of $\pi_{1}\left(P^{1}(\mathbb{C}) \backslash\left\{a_{1}, \ldots, a_{n+1}\right\}, x\right)$.

Let $\mathbb{X}=P^{1}(\mathbb{C}) \backslash\left\{a_{1}, \ldots, a_{n+1}\right\}$ and let $x \in \hat{\mathbb{X}}$. We shall describe how to choose generators of $\pi_{1}(\mathbb{X}, x)$. Let $v_{i}$ be a tangent vector in $a_{i}$. Then the loop around $a_{i}$ at the base point $v_{i}$ is the following element $S_{v_{i}}$ of $\pi_{1}\left(\mathbb{X}, v_{i}\right)$ (see picture).

For each $i$ let us choose a tangent vector $v_{i} \in T_{a_{i}}\left(P^{1}(\mathbb{C})\right)$. Let us choose a family of paths $\Gamma=\left\{\gamma_{i}\right\}_{i=1}^{n+1}$ in $J(\hat{\mathbb{X}})$ from $x$ to each $v_{i}$ such that any two paths do not intersect and no path self intersects. The indices are chosen in such a way that when we make a small circle around $x$ (around $a_{k}$ if $x$ is a tangent vector at $a_{k}$ ) in the opposite clokwise direction, starting from $\gamma_{1}$ we meet $\gamma_{2}, \gamma_{3}, \ldots, \gamma_{n+1}$. If $\gamma_{1}$ is a constant path equal $v_{1}$ we meet $\gamma_{2}, \ldots, \gamma_{n+1}$. To the path $\gamma_{i}$ we associate the following element $S_{i}$ in $\pi_{1}(\mathbb{X}, x)$. We 
move along $\gamma_{i}$, we make the loop $S_{v_{i}}$ around $a_{i}$ and we veturn along $\gamma_{i}^{-1}$ to $x$. If $\gamma_{1}$ is a constant path equal $v_{1}$ then $S_{1}$ is $S_{v_{1}}$.

The following lemma is obvious.

Lemma 8.1. The elements $S_{1}, S_{2}, \ldots, S_{n+1}$ are generators of $\pi_{1}(\mathbb{X}, x)$. We have $S_{n+1}$. $\ldots \cdot S_{2} \cdot S_{1}=1$.

Definition 8.2. The ordered sequence $\left(S_{1}, \ldots, S_{n+1}\right)$ of elements of $\pi_{1}(\mathbb{X}, x)$ obtained from the family of paths $\Gamma=\left\{\gamma_{i}\right\}_{i=1}^{n+1}$ we shall call a sequence of geometric generators of $\pi_{1}(\mathbb{X}, x)$ associated to $\Gamma=\left\{\gamma_{i}\right\}_{i=1}^{n+1}$.

\section{$\S$ 9. Monodromy of iterated integrals on $P^{1}(\mathbb{C}) \backslash\left\{a_{1}, \cdots, a_{n+1}\right\}$.}

Let $X=P^{1}(\mathbb{C}) \backslash\left\{a_{1}, \cdots, a_{n+1}\right\}$. Let $x_{1}, x_{2}, x_{3} \in \hat{X}$ and let $z_{0} \in X$. Let $\gamma_{i}$ for $i=1,2,3$ be a path belonging to $J(\hat{X})$ from $x_{i}$ to $z_{0}$. Let us set $\gamma_{i j}:=\gamma_{j}^{-1} \circ \gamma_{i}$.

Proposition 9.1. Let us prolongate each function $\Lambda_{x_{i}}(z)$ along $\gamma_{i}$ to the point $z_{0}$. There exist elements $a_{x_{j}}^{x_{i}}\left(\gamma_{i j}\right) \in P(X)$ such that

$$
\Lambda_{x_{i}}(z) \cdot a_{x_{j}}^{x_{i}}\left(\gamma_{i j}\right)=\Lambda_{x_{j}}(z)
$$

for all $z$ in a small neibourhood of $z_{0}$. The elements $a_{x_{j}}^{x_{i}}\left(\gamma_{i j}\right)$ satisfy the following relations

$$
\begin{aligned}
& a_{x_{i}}^{x_{i}}\left(\gamma_{i i}\right)=1, \\
& a_{x_{j}}^{x_{i}}\left(\gamma_{i j}\right) \cdot a_{x_{i}}^{x_{j}}\left(\gamma_{j i}\right)=1, \\
& a_{x_{j}}^{x_{i}}\left(\gamma_{i j}\right) \cdot a_{x_{k}}^{x_{j}}\left(\gamma_{j k}\right)=a_{x_{k}}^{x_{i}}\left(\gamma_{i k}\right) .
\end{aligned}
$$

Proof. The existence of $a_{x_{j}}^{x_{i}}\left(\gamma_{i j}\right)$ follows from the fact that $\Lambda_{x_{i}}(z)^{\prime} s$ are horizontal sections. The first two relations are obvious. The last relation follows from equalities $\Lambda_{x_{i}}(z)$. $a_{x_{j}}^{x_{i}}\left(\gamma_{i j}\right)=\Lambda_{x_{j}}(z), \Lambda_{x_{j}}(z) \cdot a_{x_{k}}^{x_{j}}\left(\gamma_{j k}\right)=\Lambda_{x_{k}}(z)$ and $\Lambda_{x_{i}}(z) \cdot a_{x_{k}}^{x_{i}}\left(\gamma_{i k}\right)=\Lambda_{x_{k}}(z)$. 
Proposition 9.2. Let $v_{k} \in T_{a_{k}} P^{1}(\mathbb{C}) \backslash\{0\}$. Let $S_{k}$ be a loop around $a_{k}$ based at $v_{k} \in$ $T_{a_{k}}\left(P^{1}(\mathbb{C})\right) \backslash\{0\}$, (see picture)

The monodromy of $\Lambda_{v_{k}}$ along $S_{k}$ is given by

$$
S_{k}: \Lambda_{v_{k}}(z) \rightarrow \Lambda_{v_{k}}(z) \cdot e^{-2 \pi i X_{k}}
$$

Proof. The monodromy of $\Lambda_{v_{k}}\left(k^{m}\right)(z):=\Lambda_{a_{k}, v_{k}}(k, k, \cdots, k)(z)$ along $S_{k}$ is given by $S_{k}: \Lambda_{v_{k}}\left(k^{m}\right)(z) \rightarrow \Lambda_{v_{k}}\left(k^{m}\right)(z)+\sum_{l=1}^{m} \Lambda_{v_{k}}\left(k^{m-l}\right)(z) \frac{(-2 \pi i)^{l}}{l !}$. This implies that the monodromy of $\Lambda_{v_{k}}\left(\alpha_{1}, \cdots, \alpha_{p}, k^{m}\right)(z)$ along $S_{k}$ is given by $S_{k}: \Lambda_{v_{k}}\left(\alpha_{1}, \cdots, \alpha_{p}, k^{m}\right)(z) \rightarrow$ $\Lambda_{v_{k}}\left(\alpha_{1}, \cdots, \alpha_{p}, k^{m}\right)(z)+\sum_{l=1}^{m} \Lambda_{v_{k}}\left(a_{1}, \cdots, a_{p}, k^{m-l}\right)(z) \frac{(-2 \pi i)^{l}}{l !}$. Hence it follows the formula for the monodromy of $\Lambda_{v_{k}}(z)$ along $S_{k}$.

Let $x \in \hat{X}$. Let us choose $v_{i} \in T_{a_{i}} P^{1}(\mathbb{C}) \backslash\{0\}$ for $i=1,2, \cdots, n+1$. Let $\left(S_{1}, \cdots, S_{n+1}\right)$ be a sequence of geometric generators of $\pi_{1}(X, x)$ associated to $\Gamma=\left\{\gamma_{1}\right\}_{i=1}^{n+1}$ where $\Gamma$ is a family of paths in $J^{\prime}(\hat{X})$ from $x$ to $v_{i}$ for $i=1,2, \cdots, n+1$.

Theorem 9.3. The monodromy of the function $\Lambda_{x}(z)$ along the loop $S_{k}$ is given by

$$
S_{k}: \Lambda_{x}(z) \rightarrow \Lambda_{x}(z) \cdot a_{v_{k}}^{x}\left(\gamma_{k}\right) \cdot e^{-2 \pi i X_{k}} \cdot\left(a_{v_{k}}^{x}\left(\gamma_{k}\right)\right)^{-1}
$$

Proof. It follows from Proposition 9.1 that

$$
\Lambda_{x}(z) \cdot a_{v_{k}}^{x}\left(\gamma_{k}\right)=\Lambda_{v_{k}}(z)
$$

for $z$ in the small neibourhood of some $\gamma\left(z_{0}\right)$. This equality is preserved after the monodromy transformation along $S_{k}$, hence we have

$$
\left(\Lambda_{x}(z)\right)^{S_{k}} \cdot a_{v_{k}}^{x}\left(\gamma_{k}\right)=\left(\Lambda_{v_{k}}(z)\right)^{S_{k}}
$$


where ( $)^{S_{k}}$ denotes the function ( ) after the monodromy transformation along $S_{k}$. If follows from Proposition 9.2 that

$$
\left(\Lambda_{v_{k}}(z)\right)^{S_{k}}=\Lambda_{v_{k}}(z) \cdot e^{-2 \pi i X_{k}}
$$

If we substitute $\left(*_{3}\right)$ in $\left(*_{2}\right)$ and then substitute $\left(*_{1}\right)$ for $\Lambda_{v_{k}}(z)$ we get the formula for $\left(\Lambda_{x}(z)\right)^{S_{k}}$.

Corollary 9.4. The monodromy of the function $L_{x}(z)$ along the loop $S_{k}$ is given by

$$
S_{k}: L_{x}(z) \rightarrow L_{v_{k}}(z) \cdot \alpha_{v_{x}}^{x}\left(\gamma_{k}\right) \cdot\left(-2 \pi i X_{k}\right) \cdot \alpha_{v_{k}}^{x}\left(\gamma_{k}\right)^{-1}
$$

where $\alpha_{v_{k}}^{x}\left(\gamma_{k}\right)=\log \left(\alpha_{v_{k}}^{x}\left(\gamma_{k}\right)\right)$.

Proof. The corollary follows immediately from Proposition 3.9. ii).

9.5 It follows from above, that the definition of the monodromy homomorphism (Definition 3.3) extends to any $x \in \hat{X}$. Hence for any $v \in \hat{X}$ we have a monodromy homomorphism

$$
\theta_{v, X}: \pi_{1}(X, v) \rightarrow P(X)
$$

and if $v, v^{\prime} \in \hat{X}$, then the homomorphisms $\theta_{v, X}$ and $\theta_{v^{\prime}, X}$ are conjugated.

Proposition 9.6. Let $f: X=P^{1}(\mathbb{C}) \backslash\left\{a_{1}, \ldots, a_{n+1}\right\} \rightarrow Y=P^{1}(\mathbb{C}) \backslash\left\{b_{1}, \ldots, b_{m+1}\right\}$ be a regular map. Then for any $v, w \in \hat{X}$, a path $\gamma$ from $v$ to $z$ and a path $\delta$ from $v$ to $w$ we have

$$
f_{*}\left(\Lambda_{X}(z ; v, \gamma)\right)=\Lambda_{Y}(f(z) ; f(v), f(\gamma))
$$

and

$$
f_{*}\left(a_{w}^{v}(\delta)\right)=a_{f(w)}^{f(v)}(f(\delta))
$$

(notation: $f(v):=f_{*}(v)$ if $v$ is a tangent vector).

Proof. The proposition follows from the definition of $\Lambda_{X}(z ; v, \gamma)$ and $a_{w}^{v}$ for tangent vectors and from Corollary 3.8 . 


\section{$\S 10$. Calculations}

Let $\mathbb{X}=\mathbb{P}^{1}(\mathbb{C}) \backslash\{0,1, \infty\}$. The forms $\frac{d z}{z}$ and $\frac{d z}{z-1}$ form a base of $A^{1}(\mathbb{X})$. Let $X:=\left(\frac{d z}{z}\right)^{*}$ and $Y:=\left(\frac{d z}{z-1}\right)^{*}$ be the dual base of $\left(A^{1}(\mathbb{X})\right)^{*}$. Let us set $Z:=-X-Y$. The group $P(\mathbb{X})$ is the group of invertible power series with a constant term equal 1 in non-commuting variables $X$ and $Y$.

Let us fix a path $\gamma_{1}=$ interval $[0,1]$ from $\overrightarrow{01}$ to $\overrightarrow{10}$. It follows from Proposition 9.1 that

$$
\Lambda_{\overrightarrow{10}}(z) \cdot a_{\overrightarrow{01}}^{\overrightarrow{10}}(X, Y)=\Lambda_{\overrightarrow{01}}(z)
$$

Let $f(z)=1-z$. It follows from Proposition 9.6 that

$$
f_{*}\left(a_{01}^{10}(X, Y)\right)=a_{10}^{01}(X, Y)
$$

Proposition 9.1 implies

$$
a_{10}^{01}(X, Y)=\left(a_{01}^{10}(X, Y)\right)^{-1}
$$

Observe that $f_{*}(X)=Y$ and $f_{*}(Y)=X$. Hence we get the Deligne formula

$$
a_{01}^{10}(X, Y)=\left(a_{01}^{10}(Y, X)\right)^{-1}
$$

(The proof of (2) given here repeats essentially the Deligne proof.)

Let us fix a path $\gamma_{\infty}=$ interval $[\infty,-\varepsilon]+$ arc from $-\varepsilon$ to $\varepsilon$ passing by $(-i) \cdot \varepsilon(\varepsilon>0)+$ interval from $\varepsilon$ to 0 ) from $\overrightarrow{\infty 0}$ to $\overrightarrow{01}$. Let $S_{0}$ (around 0$), S_{1}$ (around 1) and $S_{\infty}$ (around $\infty)$ be geometric generators of $\pi_{1}(\mathbb{X}, \overrightarrow{01})$ associated to the family $\left\{\gamma_{0}, \gamma_{1}, \gamma_{\infty}\right\}$, where $\gamma_{0}$ is the constant path equal $\overrightarrow{01}$. Then we have $S_{0} \circ S_{1} \circ S_{\infty}=1$. The monodromy of $\Lambda_{\overrightarrow{01}}(z)$ is given by the following formulas (see Theorem 9.3)

$$
\begin{aligned}
& S_{0}: \Lambda_{\overrightarrow{01}}(z) \rightarrow \Lambda_{\overrightarrow{01}}(z) \cdot e^{(-2 \pi i) X}, \\
& S_{1}: \Lambda_{\overrightarrow{01}}(z) \rightarrow \Lambda_{\overrightarrow{01}}(z) \cdot\left(a_{10}^{01}(X, Y)\right)^{-1} \cdot e^{(-2 \pi i) Y} \cdot a_{10}^{01}(X, Y), \\
& S_{\infty}: \Lambda_{\overrightarrow{01}}(z) \rightarrow \Lambda_{\overrightarrow{01}}(z) \cdot e^{-\pi i X} \cdot\left(a_{10}^{01}(Z, X)\right)^{-1} \cdot e^{(-2 \pi i) Z} \cdot\left(a_{10}^{01}(Z, X)\right) \cdot e^{\pi i X} .
\end{aligned}
$$

The monodromy along $S_{\infty}$ needs some explanations. By Theorem 9.3 it is given by the formula $S_{\infty}: \Lambda_{\overrightarrow{01}}(z) \rightarrow \Lambda_{\overrightarrow{01}}(z) \cdot\left(a_{01}^{\infty 0}(X, Y)\right)^{-1} \cdot e^{(-2 \pi i) Z} \cdot a_{01}^{\infty 0}(X, Y)$. By Proposition 
$9.1 a_{01}^{\infty 0}(X, Y)=a_{0 \infty}^{\infty 0}(X, Y) \cdot a_{01}^{0 \infty}(X, Y)$. One calculates that $a_{01}^{0 \infty}(X, Y)=e^{\pi i \cdot X}$. Let $f(z)=\frac{z-1}{z}$. Then it follows from Proposition 9.6 that $a_{10}^{01}(Z, X)=a_{0 \infty}^{\infty 0}(X, Y)$. Hence we get the formula describing the monodromy along $S_{\infty}$.

The Lie algebra $L(\mathbb{X})$ is the completion of the free Lie algebra on two generators $X$ and $Y$. Let us set $\alpha(X, Y):=\alpha_{10}^{01}(X, Y):=\log a_{10}^{01}(X, Y)$. The monodromy of $L_{\overrightarrow{01}}(z)$ is given by the following formulas (see Corollary 9.4).

$$
\begin{aligned}
& S_{0}: L_{\overrightarrow{01}}(z) \rightarrow L_{\overrightarrow{01}}(z) \cdot(-2 \pi i) X, \\
& S_{1}: L_{\overrightarrow{01}}(z) \rightarrow L_{\overrightarrow{01}}(z) \cdot \alpha(X, Y)^{-1} \cdot(-2 \pi i) Y \cdot \alpha(X, Y), \\
& S_{\infty}: L_{\overrightarrow{01}}(z) \rightarrow L_{\overrightarrow{01}}(z) \cdot(-\pi i) X \cdot \alpha(Z, X)^{-1} \cdot(-2 \pi i) Z \cdot \alpha(Z, X) \cdot(\pi i) \cdot X .
\end{aligned}
$$

We shall calcualte coefficients of $a_{10}^{01}(X, Y)$ and $\alpha(X, Y)$. If $\omega$ is a monomial in $X$ and $Y, a(\omega)$ is the coefficient at $\omega$ of $a_{10}^{01}(X, Y)$. Let $X$ be the first basic Lie element and let $Y$ be the second basic Lie element. We shall choose a base of a free Lie algebra on $X$ and $Y$ as in $[\mathrm{MKS}]$ pages $324-325$. If $\omega$ is an element of this base, let $\alpha(\omega)$ be the coefficient at $\omega$ of $\alpha(X, Y)$. It follows from the formula (1) that

$$
\begin{aligned}
& a\left(X^{n} Y\right)=(-1)^{n} \zeta(n+1), a\left(Y^{n} X\right)=(-1)^{n+1} \zeta(n+1), \\
& a\left(X^{i} Y^{j}\right)=\int_{0}^{1}\left(-\frac{d z}{z-1}\right)^{j},\left(\frac{-d z}{z}\right)^{i}, a\left(Y^{j} X^{i}\right)=\int_{0}^{1}\left(-\frac{d z}{z}\right)^{i},\left(-\frac{d z}{z-1}\right)^{j} .
\end{aligned}
$$

(If $\omega$ is a one-form then $\omega^{i}:=\omega, \omega, \cdots, \omega$-times.) It follows from (2) that $a\left(X^{i} Y^{j}\right)+$ $a\left(Y^{i} X^{j}\right)=0$. It follows from $[\mathrm{Ch}]$ that $a\left(X^{i} Y^{j}\right)+(-1)^{i+j} a\left(Y^{j} X^{i}\right)=0$. Hence we get

$$
\alpha_{i, j}:=\alpha\left((Y X) X^{i-1} Y^{j-1}\right)=(-1)^{i} a\left(X^{i} Y^{j}\right)=(-1)^{j-1} a\left(Y^{j} X^{i}\right)
$$

and

$$
\alpha\left((Y X) X^{j-1} Y^{i-1}\right)=\alpha\left((Y X) X^{i-1} Y^{j-1}\right) .
$$

Let us set $\pi^{\prime}:=[\pi(\mathbb{X}), \pi(\mathbb{X})]$ and $\pi^{\prime \prime}:=\left[\pi^{\prime}, \pi^{\prime}\right]$. It follows from (3) that the monodromy homomorphism

$$
\theta_{\overrightarrow{01}}: \pi_{1}(\mathbb{X}, \overrightarrow{01}) \rightarrow \pi_{2}(\mathbb{X}):=\pi(\mathbb{X}) / \pi^{\prime \prime}
$$


is given by

$$
\begin{aligned}
S_{0} \rightarrow & (-2 \pi i) X, \\
S_{1} \rightarrow & (-2 \pi i) Y+[-2 \pi i Y, \alpha(X, Y)] \\
& =(-2 \pi i) Y+\sum_{i=0, j=0}^{\infty}(2 \pi i) \alpha_{i+1, j+1}\left((Y X) X^{i} Y^{j+1}\right) .
\end{aligned}
$$

The formula

$$
\int_{0}^{z} F(z) \frac{d z}{z},\left(\frac{d z}{z}\right)^{n}=\sum_{i=0}^{n} \frac{(-1)^{n-i}}{(n-i) ! i !}\left(\int_{0}^{z} F(z)(\log z)^{n-i} \frac{d z}{z}\right)(\log z)^{i}
$$

implies

$$
\alpha_{n+1, m}=\frac{(-1)^{n}(-1)^{m}}{n ! m !} \int_{0}^{1}(\log (1-z))^{m}(\log z)^{n} \frac{d z}{z}
$$

\section{$\S 11$. The configuration spaces}

Let $X=P^{1}(\mathbb{C}) \backslash\left\{a_{1}, \cdots, a_{n+1}\right\}$ and $X^{\prime}=P^{1}(\mathbb{C}) \backslash\left\{a_{1}^{\prime}, \cdots, a_{n+1}^{\prime}\right\}$. If the sequences $(a, x):=$ $\left(a_{1}, \cdots, a_{n+1}, x\right)$ and $\left(a^{\prime}, x^{\prime}\right):=\left(a_{1}^{\prime}, \cdots, a_{n+1}^{\prime}, x^{\prime}\right)$ are close then the groups $\pi_{1}(X, x)$ and $\pi_{1}\left(X^{\prime}, x^{\prime}\right)$ are canonically isomorphic. We shall study how the monodromy homomorphisms $\theta_{x, a} ;=\theta_{x, X}$ and $\theta_{x^{\prime}, a^{\prime}}:=\theta_{x^{\prime}, X^{\prime}}$ from sections 3 and 9

$$
\begin{array}{cccc}
\theta_{x, a}: & \pi_{1}(X, x) & \rightarrow & \pi(X) \\
& \Downarrow & & \| \\
\theta_{x^{\prime}, a^{\prime}}: & \pi_{1}\left(X^{\prime}, x^{\prime}\right) & \rightarrow & \pi\left(X^{\prime}\right)
\end{array}
$$

depend on $a$ and $a^{\prime}$.

Let $X_{n}=\left\{\left(z_{1}, \cdots, z_{n}\right) \in \mathbb{C}^{n} \mid z_{i} \neq z_{j}\right.$ if $\left.i \neq j\right\}$. The space of global one-forms on $X_{n}$

with logarithmic singularities, $A^{1}\left(X_{n}\right)$ is spanned by $\frac{d z_{i}-d z_{j}}{z_{i}-z_{j}}$ for $i, j \in\{1,2, \cdots, n\}$ and $i<j$. Let $X_{i j}=\left(\frac{d z_{i}-d z_{j}}{z_{i}-z_{j}}\right)^{*}$ be their formal duals. We set $X_{j i}=X_{i j}=0$. Dualizing the map

$$
\bigwedge^{2}\left(A^{1}\left(X_{n}\right)\right) \rightarrow A^{1}\left(X_{n}\right) \wedge A^{1}\left(X_{n}\right)
$$

we get that $R\left(X_{n}\right)$ is generated by

$$
\left[X_{i j}, X_{i k}+X_{j k}\right] \text { with } i, j, k \text { different }
$$


and

$$
\left[X_{i j}, X_{k l}\right] \text { with } i, j, k, l \text { different. }
$$

Let $x=\left(x_{1}, \cdots, x_{n}, x_{n+1}\right) \in X_{n+1}$ be a base point. Let $p_{i}: X_{n+1} \rightarrow X_{n}$ $(i=1, \cdots, n+1)$ be a projection $p_{i}\left(z_{1}, \cdots, z_{n+1}\right)=\left(z_{1}, \cdots, \hat{z}_{i}, \cdots, z_{n+1}\right)$, let $x(i):=$ $\left(x_{1}, \cdots, x_{i-1}, x_{i+1}, \cdots, x_{n+1}\right)$ and let $X(i, x):=p_{i}^{-1}(x(i))=\mathbb{C} \backslash\left\{x_{1}, \cdots, \hat{x}_{i}, \cdots, x_{n+1}\right\}$. $\left(\hat{z}\right.$ means $z$ is omitted). Let $k_{i}: X(i, x) \rightarrow X_{n+1}$ be given by $k_{i}(z)=\left(x_{1}, \cdots, x_{i-1}, z, x_{i+1}\right.$, $\left.\cdots x_{n+1}\right)$. The inclusion $k_{i}$ induces

$$
\left(k_{i}\right)_{*}: P(X(i, x)) \rightarrow P\left(X_{n+1}\right)
$$

and

$$
\left(k_{i}\right)_{*}: \pi(X(i, x)) \rightarrow \pi\left(X_{n+1}\right)
$$

where $\left(k_{i}\right)_{*}\left(X_{j}\right)=X_{i j}$ and $X_{j}$ is the formal dual of $\frac{d z}{z-a_{j}}$ on $X(i, x)$. The map $\left(k_{i}\right)_{*}$ is injective and its image, $\left(k_{i}\right)_{*}\left(\pi(X(i, x))\right.$ is a normal subgroup of $\pi\left(X_{n+1}\right)$.

Let $x=\left(x_{1}, \cdots, x_{n}, x_{n+1}\right) \in X_{n+1}$ and $x^{\prime}=\left(x_{1}^{\prime}, \cdots, x_{n}^{\prime}, x_{n+1}^{\prime}\right) \in X_{n+1}$. Let us set $X:=X(n+1, x)$ and $X^{\prime}=X\left(n+1, x^{\prime}\right)$. We choose a family of non-intersecting paths $\gamma_{1}, \cdots, \gamma_{n}, \gamma_{n+1}$ in $\mathbb{C}$ from $x_{1}$ to $x_{1}^{\prime}, \cdots, x_{n}$ to $x_{n}^{\prime}$ and $x_{n+1}$ to $x_{n+1}^{\prime}$. We shall identify $\pi_{1}\left(X, x_{n+1}\right)$ and $\pi_{1}\left(X^{\prime}, x_{n+1}^{\prime}\right)$ in the following way. Observe that $\gamma=\left(\gamma_{1}, \cdots, \gamma_{n}, \gamma_{n+1}\right)$ is a path in $X_{n+1}$ from $x$ to $x^{\prime}$. The identification isomorphism $\gamma$ : : $\pi_{1}\left(X, x_{n+1}\right) \rightarrow$ $\pi_{1}\left(X^{\prime}, x_{n+1}^{\prime}\right)$ is the unique isomorphism making the following diagram commutative

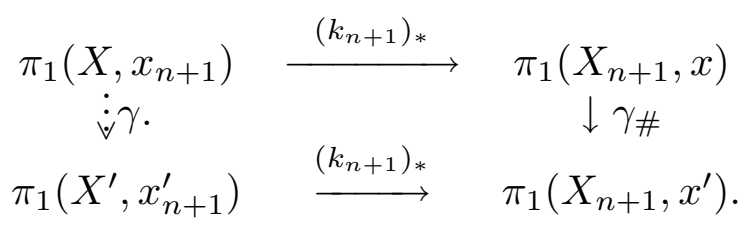

( $\gamma_{\#}$ is induced by the path $\gamma$ is a standard way).

Proposition 11.1. After the identification of the fundamental groups of $X=\mathbb{C} \backslash\left\{x_{1}, \cdots, x_{n}\right\}$ and $X^{\prime}=\mathbb{C} \backslash\left\{x_{1}^{\prime}, \cdots, x_{n}^{\prime}\right\}$ by $\gamma$. the monodromy homomorphisms

$$
\theta_{x_{n+1}, X}: \pi_{1}\left(X, x_{n+1}\right) \rightarrow \pi(X) \text { and } \theta_{x_{n+1}^{\prime}, X^{\prime}}: \pi_{1}\left(X^{\prime}, x_{n+1}^{\prime}\right) \rightarrow \pi\left(X^{\prime}\right)
$$


$\left(\pi(X)=\pi\left(X^{\prime}\right)\right)$ are conjugated by an element of the group $\pi\left(X_{n+1}\right)$. (The group $\left(k_{n+1}\right)_{*} \pi(X)$ is a normal subgroup of $\pi\left(X_{n+1}\right)$ so $\pi\left(X_{n+1}\right)$ acts on $\pi(X)$ by conjugations.)

Proof. The corollary follows from the commutative diagram

$$
\begin{aligned}
& \pi_{1}\left(X, x_{n+1}\right) \quad \stackrel{\theta_{x_{n+1}, X}}{\longrightarrow} \pi(X) \\
& \left(k_{n+1}\right)_{*} \searrow \\
& \downarrow \gamma . \quad(4) \\
& \begin{array}{c}
\pi_{1}\left(X_{n+1}, x\right) \\
\downarrow \gamma_{\#}
\end{array} \\
& \stackrel{\theta_{x, X_{n+1}}}{\longrightarrow} \\
& \left(k_{n+1}\right) * \nearrow \\
& \pi_{1}\left(X_{n+1}, x^{\prime}\right) \\
& \stackrel{\theta_{x^{\prime}, X_{n+1}}}{\longrightarrow} \\
& (2) \\
& \pi_{1}\left(X^{\prime}, x_{n+1}^{\prime}\right) \\
& \stackrel{\theta_{x_{n+1}^{\prime}}, X^{\prime}}{\longrightarrow} \\
& \searrow\left(k_{n+1}\right)_{*} \\
& \pi\left(X_{n+1}\right) \\
& \downarrow c_{L_{X_{n+1}}\left(x^{\prime} ; x, \gamma\right)} \\
& \pi\left(X_{n+1}\right) \\
& \nearrow\left(k_{n+1}\right)_{*}
\end{aligned}
$$

where $c_{L_{X_{n+1}}\left(x^{\prime} ; x, \gamma\right)}$ is a conjugation by the element $L_{X_{n+1}}\left(x^{\prime} ; x, \gamma\right)$. It follows from Proposition 3.4 that the square (1) commutes. Corollary 3.8 implies that (2) and (3) commutes. The square (4) commutes by the construction.

Corollary 11.2. Let $x=\left(x_{1}, \cdots, x_{n+1}\right) \in X_{n+1}$. Let us set $X(i):=X(i, x)$. Let $a_{i j}$ be the following element of $\pi_{1}\left(X(i), x_{i}\right)$ - a geometric generator of $\pi_{1}\left(X(i), x_{i}\right)$, which is a loop around the point $x_{j}$. Let $A_{i j}$ be its image in $\pi_{1}\left(X_{n+1}, x\right)$. Then $\theta_{x, X_{n+1}}\left(A_{i j}\right)$ is conjugated to $(-2 \pi i) X_{i j}$ in the group $\pi\left(X_{n+1}\right)$.

Proof. It follows from Proposition 9.2 that $\theta_{x_{i}, X(i)}\left(a_{i j}\right)$ is conjugated to $(-2 \pi i) X_{i j}$ in the group $\pi(X(i))$. Hence the statement follows from Corollary 3.8.

Now we shall study the relation between the monodromy representation for the configuration spaces $(\mathbb{C} \backslash\{0,1\})_{*}^{n}$ and $(\mathbb{C} \backslash\{0,1\})_{*}^{m}$. We shall use the Ihara result (see [I1] The Injectivity Theorem (i)). Let $Y_{n}:=\left(P^{1}(\mathbb{C})\right)_{*}^{n}$. The group $P G L_{2}(\mathbb{C})$ acts diagonally on $Y_{n}$ and let $\mathcal{Y}_{n}:=Y_{n} / P G L_{2}(\mathbb{C})$. Let $\psi_{k}: X_{n-1} \rightarrow \mathcal{Y}_{n}$ be the composition of the map $\left(x_{1}, \cdots, x_{k-1}, x_{k+1}, \cdots, x_{n}\right) \rightarrow\left(x_{1}, \cdots, x_{k-1}, \infty, x_{k}, \cdots, x_{n}\right)$ and the projection $Y_{n} \rightarrow \mathcal{Y}_{n}$. The map $\psi_{k}$ induces $\left(\psi_{k}\right)_{*}: H\left(X_{n-1}\right) \rightarrow H\left(\mathcal{Y}_{n}\right)$. Let us set $X_{i j}=\left(\psi_{k}\right)_{*}\left(X_{i j}\right)$ where $X_{i j}=\left(\frac{d x_{i}-d x_{j}}{x_{i}-x_{j}}\right)^{*} \in H\left(X_{n-1}\right)$. (We use the same notation for $X_{i j} \in H\left(X_{n-1}\right)$ and its image in $H\left(\mathcal{Y}_{n}\right)$. Notice also that $X_{i j}$ in $H\left(\mathcal{Y}_{n}\right)$ does not depend on the choice of $\psi_{k}$.)

Let $A_{i j} \in \pi_{1}\left(X_{n-1}, x\right)$ be such as in Corollary 11.2. The image of $A_{i j}$ in $\mathcal{Y}_{n}$ we shall also denote by $A_{i j}$. 
Corollary 11.3. The element $\theta_{y, \mathcal{Y}_{n}}\left(A_{i j}\right)$ is conjugated to $(-2 \pi i) X_{i j}$ in $\pi\left(\mathcal{Y}_{n}\right)$.

Proof. It follows from Corollary 11.2 and the commutative diagram

$$
\begin{array}{cccc}
\theta_{x, X_{n-1}}: & \pi_{1}\left(X_{n-1}, x\right) & \rightarrow & \pi\left(X_{n-1}\right) \\
& \downarrow\left(\psi_{k}\right)_{*} & & \downarrow\left(\psi_{k}\right)_{*} \\
\theta_{y, \mathcal{Y}_{n}}: & \pi_{1}\left(\mathcal{Y}_{n}, y=\psi_{k}(x)\right) & \rightarrow & \pi\left(\mathcal{Y}_{n}\right) .
\end{array}
$$

Let $\operatorname{Aut}^{*}\left(\pi\left(\mathcal{Y}_{n}\right)\right)$ be a subgroup of $\operatorname{Aut}_{\mathbb{C}}\left(\pi\left(\mathcal{Y}_{n}\right)\right)$ defined in the following way:

$$
\operatorname{Aut}^{*}\left(\pi\left(\mathcal{Y}_{n}\right)\right)=\left\{f \in \operatorname{Aut}_{\mathbb{C}}\left(\pi\left(\mathcal{Y}_{n}\right)\right) \mid \exists \alpha_{f} \in \mathbb{C}^{*}, f\left(X_{i j}\right) \sim \alpha_{f} \cdot X_{i j}\right\}
$$

$\left(\operatorname{Aut}_{\mathbb{C}}()\right.$ dentoes $\mathbb{C}$-linear automorphisms and $\sim$ means conjugated.)

Let us set

$$
T^{n}(\mathbb{C})=\left\{\varphi \in \operatorname{Hom}\left(\pi_{1}\left(\mathcal{Y}_{n, y}\right) ; \pi\left(\mathcal{Y}_{n}\right)\right) \mid \exists \alpha \in \mathbb{C}^{*}, \forall A_{i j}, \varphi\left(A_{i j}\right) \sim \alpha X_{i j}\right\}
$$

$\left(A_{i j} \in \pi_{1}\left(\mathcal{Y}_{n}, y\right)\right.$ are as in Corollary 11.3.). Observe that $T^{n}(\mathbb{C})$ is an $\operatorname{Aut}^{*}\left(\pi\left(\mathcal{Y}_{n}\right)\right)$-torsor. The subgroup of inner automorphisms $\operatorname{Inn}\left(\pi\left(\mathcal{Y}_{n}\right)\right)$ is a normal subgroup of $\operatorname{Aut}^{*}\left(\pi\left(\mathcal{Y}_{n}\right)\right)$. Hence $t^{n}(\mathbb{C}):=T^{n}(\mathbb{C}) / \operatorname{Inn}\left(\pi\left(\mathcal{Y}_{n}\right)\right)$ is a $\operatorname{Out}^{*}\left(\pi\left(\mathcal{Y}_{n}\right)\right):=\operatorname{Aut}^{*}\left(\pi\left(\mathcal{Y}_{n}\right)\right) / \operatorname{Inn}\left(\pi\left(\mathcal{Y}_{n}\right)\right)$-torsor.

The following result is an analog of the Ihara Injectivity Theorem (see [I1] page 4).

Proposition 11.4. The canonical map $\operatorname{Out}^{*}\left(\pi\left(\mathcal{Y}_{n}\right)\right) \rightarrow \operatorname{Out}^{*}\left(\pi\left(\mathcal{Y}_{n-1}\right)\right)$ is injective for $n \geq 5$.

Proof. Let $\operatorname{Out}_{1}^{*}\left(\pi\left(\mathcal{Y}_{n}\right)\right):=\operatorname{ker}\left(\operatorname{Out}^{*}\left(\pi\left(\mathcal{Y}_{n}\right)\right) \stackrel{N}{\rightarrow} \mathbb{C}^{*}\right)$, where $N(f)=\alpha_{f}$. The Lie algebra of $\operatorname{Out}^{*}\left(\pi\left(\mathcal{Y}_{n}\right)\right)$ is the Lie algebra of special derivations of $L\left(\pi\left(\mathcal{Y}_{n}\right)\right)$ modullo inner derivations. The Lie version is proved in [I1] page 12. Because $\mathrm{Out}_{1}^{*}\left(\pi\left(\mathcal{Y}_{n}\right)\right)$ is pro-nilpotent, the Lie version implies the result for $\operatorname{Out}_{1}^{*}\left(\pi\left(\mathcal{Y}_{n}\right)\right)$, and then also for $\operatorname{Out}^{*}\left(\pi\left(\mathcal{Y}_{n}\right)\right)$.

The surjective homomorphisms $\left(p_{n+1}\right)_{*}: \pi_{1}\left(\mathcal{Y}_{n+1}, y\right) \rightarrow \pi_{1}\left(\mathcal{Y}_{n}, y^{\prime}\right)$ and $\left(p_{n+1}\right)_{*}:$ $\pi\left(\mathcal{Y}_{n+1}\right) \rightarrow \pi\left(\mathcal{Y}_{n}\right)$ induce the morphism of torsors

$$
t^{n+1}(\mathbb{C}) \rightarrow t^{n}(\mathbb{C})
$$

compatible with $\operatorname{Out}^{*}\left(\pi\left(\mathcal{Y}_{n+1}\right)\right) \rightarrow \operatorname{Out}^{*}\left(\pi\left(\mathcal{Y}_{n}\right)\right)$

Lemma 11.5. The canonical morphism of torsors $t^{n+1}(\mathbb{C}) \rightarrow t^{n}(\mathbb{C})$ is injective for $n \geq 4$. This follows immediately from Proposition 11.4. 
Corollary 11.6. The monodromy homomorphism $\theta_{y, \mathcal{Y}_{n}}: \pi_{1}\left(\mathcal{Y}_{n}, y\right) \rightarrow \pi\left(\mathcal{Y}_{n}\right)$ is determined (up to conjugacy by an element of $\pi\left(\mathcal{Y}_{n}\right)$ ) by the homomorphism $\theta_{y^{\prime}, \mathcal{Y}_{4}}: \pi_{1}\left(\mathcal{Y}_{4}, y^{\prime}\right) \rightarrow$ $\pi\left(\mathcal{Y}_{4}\right)$.

Proof. Observe that $\theta_{y, \mathcal{Y}_{n}} \in t^{n}(\mathbb{C})$ and $\theta_{y^{\prime}, \mathcal{Y}_{4}}$ is the image of $\theta_{y, \mathcal{Y}_{n}}$ under the canonical morphism $t^{n}(\mathbb{C}) \rightarrow t^{4}(\mathbb{C})$.

Let $a:=\left(a_{1}, \cdots, a_{n}, a_{n+1}\right)$ be a sequence of $n+1$ different points in $P^{1}(\mathbb{C})$ and let $X_{a}:=P^{1}(\mathbb{C}) \backslash\left\{a_{1}, \cdots, a_{n}, a_{n+1}\right\}$. The vector space $H\left(X_{a}\right)$ is spanned by $X_{i}:=\left(\frac{d z}{z-a_{i}}-\right.$ $\left.\frac{d z}{z-a_{n+1}}\right)^{*} \quad i=1, \cdots, n$. Let us set $X_{n+1}:=-\sum_{i=1}^{n} X_{i}$. Let $A_{k}$ denotes a geometric generator of $X_{a}$, which is a loop around $a_{k}$. Let us set

$$
T_{a}(\mathbb{C}):=\left\{f \in \operatorname{Hom}\left(\pi_{1}\left(X_{a}, x\right) \rightarrow \pi\left(X_{a}\right)\right) \mid \exists \alpha_{f} \in \mathbb{C}^{*}, \forall A_{k} \quad f\left(A_{k}\right) \sim \alpha_{f} X_{k}\right\} .
$$

Assume that $a=\left(a_{i}\right)_{i=1}^{n+1}$ is such that $a_{1}=0, a_{2}=1, a_{3}=\infty$. The fibration

$$
X_{a} \stackrel{k_{n+2}}{\longrightarrow} \mathcal{Y}_{n+2} \stackrel{p_{n+2}}{\longrightarrow} \mathcal{Y}_{n+1} \quad\left(X_{a}=\left(p_{n+2}\right)^{-1}\left(a_{1}, \cdots, a_{n+1}\right)\right)
$$

realizes $\pi\left(X_{a}\right)$ as a normal subgroup of $\pi\left(\mathcal{Y}_{n+2}\right)\left(\left(k_{n+2}\right)_{*}\left(X_{i}\right)=X_{i, n+2}\right)$. Hence the group $\pi\left(\mathcal{Y}_{n+2}\right)$ acts on $T_{a}(\mathbb{C})$ and let

$$
t_{a}(\mathbb{C}):=T_{a}(\mathbb{C}) / \pi\left(\mathcal{Y}_{n+2}\right) .
$$

Observe that any $\pi\left(\mathcal{Y}_{n+2}\right)$-conjugate of $X_{i, n+2}$ is in the image of $\pi\left(X_{a}\right)$. Hence the restriction map

$$
\left(k_{n+2}\right)^{*}: t^{n+2}(\mathbb{C}) \rightarrow t_{a}(\mathbb{C})
$$

given by $f \rightarrow f_{\mid \pi_{1}\left(X_{a}, x\right)}$ is defined. We set

$$
\tau_{a}(\mathbb{C}):=\operatorname{im}\left(t^{n+2}(\mathbb{C}) \rightarrow t_{a}(\mathbb{C})\right) .
$$

Observe that the diagram

$$
\begin{array}{ccc}
t^{n+2}(\mathbb{C}) & \stackrel{\left(k_{n+2}\right)^{*}}{\longrightarrow} & \tau_{a}(\mathbb{C}) \\
\check{\downarrow} p r & & \downarrow p r_{1} \\
t^{4}(\mathbb{C}) & \stackrel{\left(k_{4}\right)_{*}}{\rightleftharpoons} & \tau_{0,1, \infty}(\mathbb{C})
\end{array}
$$

commutes where the map $p r_{1}$ is induced by the inclusion $X_{a} \hookrightarrow P^{1}(\mathbb{C}) \backslash\{0,1, \infty\}$. The map $\left(k_{4}\right)_{*}$ is bijective because $\mathcal{Y}_{4}=P^{1}(\mathbb{C}) \backslash\{0,1, \infty\}$. Lemma 11.5 implies that the map $p r$ is injective. Hence both maps, $\left(k_{n+2}\right)^{*}$ and $p r_{1}$ are injective. Hence we have proved the following result. 
Proposition 11.7. i) The $\pi\left(\mathcal{Y}_{n+2}\right)$-conjugacy class of the monodromy homomorphism $\theta_{x, \mathcal{Y}_{n+2}}: \pi_{1}\left(\mathcal{Y}_{n+2}, x\right) \rightarrow \pi\left(\mathcal{Y}_{n+2}\right)$ is determined by its restriction to $\pi_{1}\left(X_{a}, x^{\prime}\right)$.

ii) The $\pi\left(\mathcal{Y}_{n+2}\right)$-conjugacy class of the monodromy homomorphism $\theta_{x, X_{a}}: \pi_{1}\left(X_{a}, x\right) \rightarrow$ $\pi\left(X_{a}\right)$ is determined by the monodromy homomorphism

$$
\theta_{x^{\prime}, P^{1}(\mathbb{C}) \backslash\{0,1, \infty\}}: \pi_{1}\left(P^{1}(\mathbb{C}) \backslash\{0,1, \infty\}, x^{\prime}\right) \rightarrow \pi\left(P^{1}(\mathbb{C}) \backslash\{0,1, \infty\}\right) .
$$

\section{$\S 12$. The Drinfeld-Ihara $Z / 5$-cycle relation}

In this section we show that the element which describes the monodromy of all iterated integrals on $P^{1}(\mathbb{C}) \backslash\{0,1, \infty\}$ satisfies the Drinfeld-Ihara relation.

\subsection{Configuration spaces}

If $T$ is a topological space we set $T_{*}^{n}=\left\{\left(t_{1}, \cdots, t_{n}\right) \in T^{n} \mid t_{i} \neq t_{j}\right.$ if $\left.i \neq j\right\}$. The group $\Sigma^{n}$ acts on $T_{*}^{n}$ by permutations.

Let us set $Y_{n}=\left(P^{1}(\mathbb{C})\right)_{*}^{n}$ and $\mathcal{Y}_{n}=\left(P^{1}(\mathbb{C}) \backslash\{0,1, \infty\}\right)_{*}^{n-3}$. Let $a, b, c \in P^{1}(\mathbb{C})$ be three different points and let $\varphi_{a, b, c}(z)=\frac{b-c}{b-a} \cdot \frac{z-a}{z-c}$. The map $\Phi_{4,5}: Y_{5} \rightarrow \mathcal{Y}_{5}$ given by $\Phi_{4,5}\left(x_{1}, x_{2}, x_{3}, x_{4}, x_{5}\right)=\left(\varphi_{x_{1}, x_{2}, x_{3}}\left(x_{4}\right), \varphi_{x_{1}, x_{2}, x_{3}}\left(x_{5}\right)\right)$ induces a bijection

$$
\varphi_{4,5}: Y_{5} / \mathrm{PGL}_{2}(\mathbb{C}) \rightarrow \mathcal{Y}_{5}
$$

The action of $\Sigma_{5}$ on $Y_{5}$ induces an action of $\Sigma_{5}$ on $\mathcal{Y}_{5}$. The map $\sigma: \mathcal{Y}_{5} \rightarrow \mathcal{Y}_{5}, \sigma(s, t)=$ $\left(\frac{t-1}{t-s}, \frac{1}{s}\right)$ corresponds to the permutation $\tilde{\sigma}$ of $Y_{s}$ given by

$$
\tilde{\sigma}\left(x_{1}, x_{2}, x_{3}, x_{4}, x_{5}\right)=\left(x_{2}, x_{3}, x_{4}, x_{5}, x_{1}\right) .
$$

Observe that the points $A=\left(\frac{\sqrt{5}-1}{2}, \frac{\sqrt{5}+1}{2}\right) \in \mathcal{Y}_{5}$ and $B=\left(\frac{-\sqrt{5}-1}{2}, \frac{-\sqrt{5}+1}{2}\right) \in$ $\mathcal{Y}_{5}$ are fixed by $\sigma$.

The one-forms $\frac{d s}{s}, \frac{d s}{s-1}, \frac{d t}{t}, \frac{d t}{t-1}, \frac{d s-d t}{s-t}$ generate $A^{1}\left(\mathcal{Y}_{5}\right)$ and $H_{\mathrm{DR}}^{1}\left(\mathcal{Y}_{5}\right)$. Let $S_{0}, S_{1}, T_{0}, T_{1}$ and $N$ be their formal duals. The subspace $R\left(\mathcal{Y}_{5}\right)$ of $H\left(\mathcal{Y}_{5}\right)^{\otimes 2}$ is generated by

$$
\begin{aligned}
& {\left[S_{i}, N\right]+\left[T_{i}, N\right] \quad i=0,1} \\
& {\left[S_{i}, T_{i}\right]+\left[S_{i}, N\right] \quad i=0,1} \\
& {\left[T_{i}, S_{i}\right]+\left[T_{i}, N\right] \quad i=0,1} \\
& {\left[S_{0}, T_{1}\right] \quad \text { and } \quad\left[S_{1}, T_{0}\right]}
\end{aligned}
$$


where $[A, B]=A \otimes B-B \otimes A$.

Let $G:=P\left(\mathcal{Y}_{5}\right)$ i.e. $G$ is a multiplicative group of the algebra of formal power series in non-commuting variables $S_{0}, S_{1}, T_{0}, T_{1}$ and $N$ divided by the ideal generated by $R\left(\mathcal{Y}_{5}\right)$.

The principal fibration

$$
\mathcal{Y}_{5} \times G \rightarrow \mathcal{Y}_{5}
$$

we equipped with the integrable connection given by the one form

$$
\begin{aligned}
\omega_{\mathcal{Y}_{5}}= & \left(\frac{d t}{t-1}-\frac{d t}{t}\right) \otimes T_{1}+\left(-\frac{d t}{t}\right) \otimes T_{\infty} \\
& +\left(\frac{d s-d t}{s-t}-\frac{d t}{t}\right) \otimes N+\frac{d s}{s} \otimes S_{0}+\frac{d s}{s-1} \otimes S_{1}
\end{aligned}
$$

where $T_{\infty}=-T_{0}-T_{1}-N$. We shall write shortly $\omega$ instead of $\omega_{\mathcal{Y}_{5}}$.

\subsection{Integration of $w$}

We recall that on $P^{1}(\mathbb{C}) \backslash\{0,1, \infty\}$ we have

$$
\Lambda_{\underset{\infty 1}{\longrightarrow}}(z) \cdot a_{\overrightarrow{1 \infty}}^{\overrightarrow{\infty 1}}=\Lambda_{\overrightarrow{1 \infty}}(z) \quad(\text { see Proposition 9.1) }
$$

The monodromy of $\Lambda_{\infty 1}(z)$ is given by:

$$
\begin{aligned}
& (\text { around } \infty): \Lambda_{\overrightarrow{\infty 1}}(z) \rightarrow \Lambda_{\overrightarrow{\infty 1}}(z) \cdot e^{-2 \pi i T_{\infty}} \\
& \left(\text { around 1) }: \Lambda_{\overrightarrow{\infty 1}}(z) \rightarrow \Lambda_{\overrightarrow{\infty 1}}(z) \cdot a_{1 \infty}^{\infty 1} \cdot e^{-2 \pi i T_{1}} \cdot\left(a_{1 \infty}^{\infty 1}\right)^{-1}\right.
\end{aligned}
$$

(see Theorem 9.3). We have $f_{*}\left(a_{10}^{01}\left(T_{0}, T_{1}\right)\right)=a_{1 \infty}^{\infty 1}\left(T_{\infty}, T_{1}\right)$ where $f_{*}\left(T_{0}\right)=T_{\infty}, f_{*}\left(T_{1}\right)=$ $T_{1}$ and $f(z)=1 / z$.

We have assymptotically at $\infty$

$$
\begin{aligned}
& \Lambda_{\infty 1}(z) \underset{z=\infty}{\sim} e^{\left(\int_{1}^{z} \frac{d t}{t}\right) T_{\infty}} \\
& \text { i.e. } \lim _{\substack{z \rightarrow \infty \\
z>1}}\left(\Lambda_{\infty 1}(z) \cdot e^{-\left(\int_{1}^{z} \frac{d t}{t}\right) T_{\infty}}\right)=1 .
\end{aligned}
$$

Let $P_{\varepsilon}=(\varepsilon, 1+\varepsilon) \in \mathcal{Y}_{5}$ where $\varepsilon>0$ and small. Let $\Lambda_{P_{\varepsilon}}(z ;$ path) be a horizontal section of $\omega$ such that $\Lambda_{P_{\varepsilon}}\left(P_{\varepsilon}\right)=1$. Let $\gamma$ be a path in $\mathcal{Y}_{5}$ from $P_{\varepsilon}$ to $\sigma\left(P_{\varepsilon}\right)=(\varepsilon, 1 / \varepsilon)$ which is constant $(=\varepsilon)$ on the first coordinate. 
Assuming $s=$ constant $(=\varepsilon)$ we have

$$
\Lambda_{P_{\varepsilon}}(z) \cdot \underset{1 \infty}{\stackrel{1+\varepsilon}{\longrightarrow}}=\Lambda_{\underset{1 \infty}{\longrightarrow}}(z)
$$

Hence we have assymptotically for positive, small $\varepsilon$

$$
a_{\overrightarrow{1 \infty}}^{1+\varepsilon} \underset{\varepsilon=0}{\sim} e^{\left(-\int_{\infty}^{1+\varepsilon}\left(\frac{d t}{t-1}-\frac{d t}{t}\right) T_{1}\right)}
$$

It follows from 12.2.0, 12.2.1 and 12.2.2 and Proposition 9.1 that for $\varepsilon$ positive, near 0

$$
\Lambda_{P_{\varepsilon}}\left(\sigma\left(P_{\varepsilon}\right) ; \gamma\right) \underset{\varepsilon=0}{\sim} e^{\left(\int_{1}^{1 / \varepsilon} \frac{d z}{z}\right) T_{\infty}} \cdot a_{1 \infty}^{\infty 1}\left(T_{\infty}, T_{1}\right) \cdot e^{\left(\int_{\infty}^{1+\varepsilon} \frac{d t}{t-1}-\frac{d t}{t}\right) T_{1}}
$$

Let $p=\gamma+\sigma(\gamma)+\sigma^{2}(\gamma)+\sigma^{3}(\gamma)+\sigma^{4}(\gamma)$. Then $\Lambda_{P_{\varepsilon}}\left(P_{\varepsilon} ; p\right)=1$ because the path $p$ is contractible in $\mathcal{Y}_{5}$. On the other hand

$$
\begin{aligned}
1=\Lambda_{P_{\varepsilon}}\left(P_{\varepsilon}, p\right)=\Lambda_{\sigma^{4}\left(P_{\varepsilon}\right)}\left(P_{\varepsilon} ; \sigma^{4}(\gamma)\right) \cdot \Lambda_{\sigma^{3}\left(P_{\varepsilon}\right)}\left(\sigma^{4}\left(P_{\varepsilon}\right) ; \sigma^{3}(\gamma)\right) \\
\cdot \Lambda_{\sigma^{2}\left(P_{\varepsilon}\right)}\left(\sigma^{3}\left(P_{\varepsilon}\right) ; \sigma^{2}(\gamma)\right) \cdot \Lambda_{\sigma\left(P_{\varepsilon}\right)}\left(\sigma^{2}\left(P_{\varepsilon}\right) ; \sigma(\gamma)\right) \cdot \Lambda_{P_{\varepsilon}}\left(\sigma\left(P_{\varepsilon}\right) ; \gamma\right)
\end{aligned}
$$

The formula

$$
\left(\sigma^{i}\right)_{*}\left(\Lambda_{P_{\varepsilon}}\left(\sigma\left(P_{\varepsilon}\right), \gamma\right)\right)=\Lambda_{\sigma^{i}\left(P_{\varepsilon}\right)}\left(\sigma^{i+1}\left(P_{\varepsilon}\right), \sigma^{i}(\gamma)\right)
$$

(see Corollary 3.8) implies that

$$
1=\sigma_{*}^{4}(L) \cdot \sigma_{*}^{3}(L) \cdot \sigma_{*}^{2}(L) \cdot \sigma_{*}(L) \cdot L
$$

where $L=\Lambda_{P_{\varepsilon}}\left(\sigma\left(P_{\varepsilon}\right), \gamma\right)$. Let

$$
\mathrm{E}=e^{\left(\int_{1}^{1 / \varepsilon} \frac{d z}{z}\right) T_{\infty}} \cdot a_{1 \infty}^{\infty 1}\left(T_{\infty}, T_{1}\right) \cdot e^{\left(\int_{\infty}^{1+\varepsilon} \frac{d t}{t-1}-\frac{d t}{t}\right) T_{1}}
$$

It follows from 12.2.3 that

$$
1 \underset{\varepsilon=0}{\sim} \sigma_{*}^{4}(\mathrm{E}) \cdot \sigma_{*}^{3}(\mathrm{E}) \cdot \sigma_{*}^{2}(\mathrm{E}) \cdot \sigma_{*}(\mathrm{E}) \cdot \mathrm{E} .
$$


The factors $e^{\int_{\infty}^{1+\varepsilon}\left(\frac{d t}{t-1}-\frac{d t}{t}\right) T_{\infty}\left(=\sigma_{*}^{2}\left(T_{1}\right)\right)}$ and $e^{\left(\int_{1}^{1 / \varepsilon} \frac{d z}{z}\right) T_{\infty}}$ can be placed together in the product $\sigma_{*}^{4}(\mathrm{E}) \cdot \ldots \mathrm{E}$ because $T_{\infty}=\sigma_{*}^{2}\left(T_{1}\right)$ commutes with $\sigma_{*}\left(T_{1}\right)=S_{0}$ and $\sigma_{*}\left(T_{\infty}\right)=S_{1}$. After the calculations we get

$$
\int_{\infty}^{1+\varepsilon}\left(\frac{d t}{t-1}-\frac{d t}{t}\right)-\int_{1}^{1 / \varepsilon} \frac{d t}{t}=-\log (1+\varepsilon)
$$

Repeating the same argument for $S_{1}, S_{1}+T_{1}+N, T_{1}$ and $S_{0}$ and passing to the limit with $\varepsilon$ we get

$$
\sigma_{*}^{4}(\mathfrak{a}) \cdot \sigma_{*}^{3}(\mathfrak{a}) \cdot \sigma_{*}^{2}(\mathfrak{a}) \cdot \sigma_{*}(\mathfrak{a}) \cdot \mathfrak{a}=1
$$

where $\mathfrak{a}=a_{1 \infty}^{\infty 1}\left(T_{\infty}, T_{1}\right)$. The last formula we can write in the form

$$
\mathfrak{a}\left(S_{0}, S_{1}+T_{1}+N\right) \cdot \mathfrak{a}\left(T_{1}, S_{1}\right) \cdot \mathfrak{a}\left(S_{1}+T_{1}+N, T_{\infty}\right) \cdot \mathfrak{a}\left(S_{1}, S_{0}\right) \cdot \mathfrak{a}\left(T_{\infty}, T_{1}\right)=1
$$

because $\sigma_{*}\left(S_{0}\right)=T_{\infty}, \sigma_{*}\left(S_{1}\right)=S_{1}+T_{1}+N, \sigma_{*}\left(T_{0}\right)=N, \sigma_{*}\left(T_{1}\right)=S_{0}$ and $\sigma_{*}(N)=$ $-S_{0}-S_{1}-N$.

Let $\psi_{5}: \mathbb{C}_{*}^{4} \rightarrow \mathcal{Y}_{5}$ be given by $\psi_{5}\left(z_{1}, z_{2}, z_{3}, z_{4}\right)=\Phi_{4,5}\left(z_{1}, z_{2}, z_{3}, z_{4}, \infty\right)$. Let $\left(A_{i j}\right)_{i, j}$ be formal duals of $\left(\frac{d z_{i}-d z_{j}}{z_{i}-z_{j}}\right)_{i, j}$. Then we have

$$
\begin{aligned}
& \psi_{5 *}\left(A_{12}\right)=-S_{0}-S_{1}-T_{0}-T_{1}-N, \\
& \psi_{5 *}\left(A_{13}\right)=S_{1}+T_{1}+N, \\
& \psi_{5 *}\left(A_{14}\right)=S_{0}, \\
& \psi_{5 *}\left(A_{23}\right)=S_{0}+T_{0}+N, \\
& \psi_{5 *}\left(A_{24}\right)=S_{1}, \\
& \psi_{5 *}\left(A_{34}\right)=-S_{0}-S_{1}-N .
\end{aligned}
$$

Using $\psi_{1}: \mathbb{C}_{*}^{4} \rightarrow \mathcal{Y}_{5}$ given by $\psi_{1}\left(z_{2}, z_{3}, z_{4}, z_{5}\right)=\Phi_{4,5}\left(\infty, z_{2}, z_{3}, z_{4}, z_{5}\right)$ we get

$$
\begin{aligned}
& \psi_{1 *}\left(A_{23}\right)=S_{0}+T_{0}+N, \\
& \psi_{1 *}\left(A_{24}\right)=S_{1}, \\
& \psi_{1 *}\left(A_{25}\right)=T_{1}, \\
& \psi_{1 *}\left(A_{34}\right)=-S_{0}-S_{1}-N, \\
& \psi_{1 *}\left(A_{35}\right)=-T_{0}-T_{1}-N, \\
& \psi_{1 *}\left(A_{45}\right)=N .
\end{aligned}
$$


We set $X_{i j}:=\psi_{\varepsilon *}\left(A_{i j}\right) \varepsilon=1,5$. Then $X_{15}=T_{0}$. Hence finally we get a formula

$$
\mathfrak{a}\left(X_{14}, X_{13}\right) \cdot \mathfrak{a}\left(X_{25}, X_{24}\right) \cdot \mathfrak{a}\left(X_{13}, X_{35}\right) \cdot \mathfrak{a}\left(X_{24}, X_{14}\right) \cdot \mathfrak{a}\left(X_{35}, X_{25}\right)=1 .
$$

If we use $\Phi_{2,4}: X_{*}^{5} \rightarrow \mathcal{Y}_{5}$ given by $\Phi_{2,4}(0, s, 1, t, \infty)=(s, t)$ and repeat the calculations in $\mathcal{Y}_{5}$ we get the same formula as before, but the $X_{i j}$ 's names of $S_{0}, S_{1}, \ldots$ are now different and the resulting formula is:

$$
\mathfrak{a}\left(X_{12}, X_{15}\right) \cdot \mathfrak{a}\left(X_{34}, X_{23}\right) \cdot \mathfrak{a}\left(X_{15}, X_{45}\right) \cdot \mathfrak{a}\left(X_{23}, X_{12}\right) \cdot \mathfrak{a}\left(X_{45}, X_{34}\right)=1
$$

This is exactly the formula which appears in [12] page 106 if we replace $\mathfrak{a}()$ by $\mathfrak{a}()^{-1}$.

Proposition 12.3. For any permutation $\sigma$ of five letters we have

$$
\begin{gathered}
\mathfrak{a}\left(X_{\sigma(14)}, X_{\sigma(13)}\right) \cdot \mathfrak{a}\left(X_{\sigma(25)}, X_{\sigma(24)}\right) \cdot \mathfrak{a}\left(X_{\sigma(13)}, X_{\sigma(35)}\right) \\
\cdot \mathfrak{a}\left(X_{\sigma(24)}, X_{\sigma(14)}\right) \cdot \mathfrak{a}\left(X_{\sigma(35)}, X_{\sigma(25)}\right)=1, \\
\mathfrak{a}\left(X_{\sigma(12)}, X_{\sigma(15)}\right) \cdot \mathfrak{a}\left(X_{\sigma(34)}, X_{\sigma(23)}\right) \cdot \mathfrak{a}\left(X_{\sigma(15)}, X_{\sigma(45)}\right) \\
\cdot \mathfrak{a}\left(X_{\sigma(23)}, X_{\sigma(12)}\right) \cdot \mathfrak{a}\left(X_{\sigma(45)}, X_{\sigma(34)}\right)=1,
\end{gathered}
$$

where $\sigma(i j)=\sigma(i) \sigma(j)$.

Proof. It follows from 12.2.4, 12.2.5 and Corollary 3.8.

Remark. The formulas of Proposition 12.3 are in the group $P\left(\mathcal{Y}_{5}\right)$. If we apply $\log$ we get formulas in the group $\pi\left(\mathcal{Y}_{5}\right)$.

In the sequel we shall work in the group $\pi\left(\mathcal{Y}_{5}\right)$.

We finish this section with a formula from which the Deligne $Z / 3$-cycle relation can be obtained. The proof is an imitation of the Deligne proof.

Proposition 12.4. Let $\alpha:=\log \mathfrak{a}$. In the group $\pi\left(\mathcal{Y}_{5}\right)$ we have

$$
\alpha\left(X_{25}, X_{23}\right)\left(-\pi i X_{23}\right) \alpha\left(X_{23}, X_{35}\right)\left(-\pi i X_{35}\right) \alpha\left(X_{35}, X_{25}\right)\left(-\pi i X_{25}\right)=-\pi i X_{14}
$$

and

ii) $\quad \alpha\left(X_{25}, X_{23}\right)\left(\pi i X_{23}\right) \alpha\left(X_{23}, X_{35}\right)\left(\pi i X_{35}\right) \alpha\left(X_{35}, X_{25}\right)\left(\pi i X_{25}\right)=\pi i X_{14}$. 
Proof. Let $\tilde{\sigma}\left(x_{1}, x_{2}, x_{3}, x_{4}, x_{5}\right)=\left(x_{1}, x_{5}, x_{2}, x_{4}, x_{3}\right)$. Then the induced map $\sigma: \mathcal{Y}_{5} \rightarrow \mathcal{Y}_{5}$ is given by $\sigma(s, t)=\left(\frac{t-1}{t} \cdot \frac{s}{s-1}, \frac{t-1}{t}\right)$ and $\sigma^{2}(s, t)=\left(\frac{s}{s-t}, \frac{1}{1-t}\right)$. Let $P_{-}=(r, 1-r)$ and $P_{+}=(r, 1+r)$ where $r$ is positive and small. Let $\mathbb{Q}_{-}=(-r, 1-r)$ and $\mathbb{Q}_{+}=(-r, 1+r)$. Let $\gamma$ be a path from $P_{+}=(r, 1+r)$ to $\sigma^{2}\left(\mathbb{Q}_{-}\right)=(r, 1 / r)$, which is constant on the first coordinate. Let $\gamma^{\prime}$ be a path from $\mathbb{Q}_{+}$to $\sigma^{2}\left(P_{-}\right)$passing through the point $\left(\frac{r}{2 r-1}, 1+r\right)$ which is piecewise constant, first on the second coordinate, next on the first coordinate.

Let $S$ be a path $[0, \pi] \ni \varphi \rightarrow\left(r, 1+r e^{i(\varphi+\pi)}\right)$ and let $S^{\prime}$ be a path $[0, \pi] \ni \varphi \rightarrow$ $\left(-r, 1+r e^{i(\varphi+\pi)}\right)$. Let us consider the composition $p=\sigma\left(\gamma^{\prime}\right) \circ \sigma\left(S^{\prime}\right) \circ \sigma^{2}(\gamma) \circ \sigma^{2}(S) \circ \gamma^{\prime} \circ$ $S^{\prime} \circ \sigma(\gamma) \circ \sigma(S) \circ \sigma^{2}\left(\gamma^{\prime}\right) \circ \sigma^{2}\left(S^{\prime}\right) \circ \gamma \circ S$. If we integrate the form $\omega$ along this path and pass to the limit if $r \rightarrow 0$ we get the square of the left hand side of the expression i).

Let $\alpha$ be a loop in the opposite clockwise direction around $(0,0)$ in the plane $P=$ $\left\{(s, t) \in \mathbb{C}^{2} \mid \alpha s+\beta t=0\right\}$. The integration of the form $\omega$ along $\alpha$ gives $(-2 \pi i)\left(S_{0}+N+\right.$ $\left.T_{0}\right)=(-2 \pi i) X_{23}$. In the model of $Y_{*}^{5} / P G L_{2}(\mathbb{C})$ in which the subspace $\left\{\left(x_{1}, x_{2}, x_{3}, x_{4}, x_{5}\right) \mid\right.$ $\left.x_{1}=x_{4}\right\}$ of $\left(P^{1}(\mathbb{C})\right)^{5}$ degenerates to a point (for example for $\Phi_{2,5}(0, s, 1, \infty, t)=(s, t)$ ), the path $p$ is homotopic to a loop around one of the points $(0,0),(1,1)$ or $(\infty, \infty)$ in the plane passing through the corresponding point $(0,0),(1,1)$ or $(\infty, \infty)$ (the point $(1,1)$ in the case of the model $\left.\Phi_{2,5}\right)$. Hence the left hand side of the expression i) is also $(-2 \pi i) \cdot X_{14}$. The proof of the second equality is similar.

Corollary 12.5. For any permutation $\sigma$ of five letters $1,2,3,4,5$ we have formulas $i^{\prime}$ ) and $\left.i i^{\prime}\right)$, which are obtained from formulas i) and ii) by replacing indices $1,2,3,4,5$ by $\sigma(1)$, $\sigma(2), \sigma(3), \sigma(4), \sigma(5)$.

Proof. One consider the map of $Y_{5}$ given by $\left(x_{i}\right)_{i=1, \ldots, 5} \rightarrow\left(x_{\sigma(i)}\right)_{i=1, \ldots, 5}$. The induced map $\sigma: \mathcal{Y}_{5} \rightarrow \mathcal{Y}_{5}$ satisfies $\sigma^{*} \omega=\sigma_{*} \omega$. This implies formulas $\left.\mathrm{i}^{\prime}\right)$ and $\left.\mathrm{ii}^{\prime}\right)$.

Remark. We have $X_{23}+X_{25}+X_{35}=X_{14}$ in the Lie algebra Lie $\left(\mathcal{Y}_{5}\right)$. If we set $X_{14}=0$ then the formulas i) and ii) reduce to the Deligne formula. 


\section{$\S 13$. Functional equations of iterated integrals.}

In this section we give necessary and sufficient conditions to have a functional equation of iterated integrals in terms of exotic analogues of the Bloch group. We shall work only on a pointed projective line.

Let $\operatorname{Lie}\left(\mathbb{C}(z)^{*}\right)$ be a free Lie algebra on the abelian group $\mathbb{C}(z)^{*}$. Let $I \subset \operatorname{Lie}\left(\mathbb{C}(z)^{*}\right)$ be a Lie ideal consisting of all brackets $\left[\ldots\left[f_{1}, f_{2}\right] \ldots\left[\ldots, f_{k}\right] \ldots\right]$ such that at least one $f_{i}$ is in $\mathbb{C}^{*}$. Observe that $L\left(\mathbb{C}(z)^{*}\right)$ is a free Lie algebra on the set $\{(z-a) \mid a \in \mathbb{C}\}$.

Let $\mathbb{X}=\mathbb{P}^{1}(\mathbb{C}) \backslash\left\{a_{1}, \ldots, a_{n}, \infty\right\}$ and let $\mathbb{Y}=\mathbb{P}^{1}(\mathbb{C}) \backslash\left\{b_{1}, \ldots, b_{m}, \infty\right\}$. We set

$$
\mathcal{B}(\mathbb{Y}):=\underset{f \in \mathbb{C}(z) \backslash\left\{b_{1}, \ldots, b_{m}\right\}}{\oplus} \mathbb{Z}
$$

the free abelian group on the set $\mathbb{C}(z) \backslash\left\{b_{1}, \ldots, b_{m}\right\}$. The generator corresponding to $f$ we shall denote by $[f]$. For any homogenous element $e \in \operatorname{Lie}(H(\mathbb{Y}))$,

$$
e=\sum_{i} \alpha_{i}\left[\ldots\left[B_{i_{1}} \ldots\left[\ldots, B_{i_{k}}\right] \ldots\right]\right.
$$

where $B_{j}=\left(\frac{d z}{z-b_{j}}\right)^{*}$ we define a map

$$
b_{Y}(e): \mathcal{B}(\mathbb{Y}) \rightarrow L\left(\mathbb{C}(z)^{*}\right)
$$

by the formula

$$
b_{Y}(e)([f])=\sum_{i} \alpha_{i}\left[\ldots\left[f-b_{i_{1}}, \ldots\left[\ldots, f-b_{i_{k}}\right] \ldots\right]\right.
$$

Let us fix an ordering $B_{1}=\left(\frac{d z}{z-b_{1}}\right)^{*}, B_{2}, \ldots, B_{m}$ of the base of $H(\mathbb{Y})$. Then there is a canonical base $\mathcal{B}=\left\{e_{i}\right\}_{i \in I}$ of $\operatorname{Lie}(H(\mathbb{Y}))$ given by basic Lie elements (see [MKS]). Let $\left\{e_{i}^{*}\right\}_{i \in I}$ be the dual linear forms. 
Theorem13.1. Let $e_{1}, \ldots, e_{n} \in \mathcal{B}$ be basic Lie elements of degree $n$. Let $f_{1}, \ldots, f_{n}: \mathbb{X} \rightarrow$ $\mathbb{Y}$ be regular maps. Let $n_{1}, \ldots, n_{N}$ be integers. Let $\gamma$ be a path in $\mathbb{X}$ from $x$ to $z$. The following conditions are equivalent.

i)

$$
\begin{aligned}
& \sum_{i=1}^{N} n_{i} \cdot e_{i}^{*}\left(L_{\mathbb{Y}}\left(f_{i}(z) ; f_{i}(x), f_{i}(\gamma)\right)=0\right. \\
& \sum_{i=1}^{N} n_{i} \cdot e_{i}^{*} \circ\left(f_{i}\right)_{*}=0 \quad \text { in } \quad \operatorname{Hom}\left(\Gamma^{n} \pi(\mathbb{X}) / \Gamma^{n+1} \pi(\mathbb{X}) ; \mathbb{C}\right) ; \\
& \sum_{i=1}^{N} n_{i} \cdot b_{\mathbb{Y}}\left(e_{i}\right)\left(\left[f_{i}\right]\right)=0 .
\end{aligned}
$$

Proof. It follows from Corollary 2.2 in [W4] that conditions i) and ii) are equivalent. Hence we must show that ii) and iii) are equivalent. Let $e=\left[\ldots\left[B_{i_{1}}, \ldots\left[\ldots, B_{i_{k}}\right] \ldots\right]\right.$ be a basic Lie element of degree $n$ and let $e^{*}$ be its dual. Let $f: \mathbb{X} \rightarrow \mathbb{Y}$ be a regular map. The map $f$ and $e^{*}$ induce

$$
\operatorname{Lie}(H(\mathbb{X})) \stackrel{f_{*}}{\longrightarrow} \operatorname{Lie}(H(\mathbb{Y})) \stackrel{e_{*}}{\longrightarrow} \mathbb{C} .
$$

Let us set $\mathbb{C}^{\vee}:=\operatorname{Hom}(\mathbb{C}, \mathbb{C})$. Passing to dual objects we get a map

$$
\operatorname{Lie}\left(A^{1}(\mathbb{X})\right) \stackrel{f^{*}}{\longleftarrow} \operatorname{Lie}\left(A^{1}(\mathbb{Y})\right) \stackrel{\left(e^{*}\right)^{\vee}}{\longleftarrow} \mathbb{C}^{\vee} .
$$

Observe that the condition ii) is equivalent to the condition

$$
\sum_{i=1}^{N} n_{i} \cdot\left(f_{i}\right)^{*} \circ\left(e_{i}^{*}\right)^{\vee}=0 \quad \text { in } \operatorname{Hom}\left(\mathbb{C}^{\vee}, \operatorname{Lie}\left(A^{1}(\mathbb{X})\right)\right) .
$$

Observe that $f^{*}\left(\frac{d z}{z-b_{i}}\right)=\frac{f^{\prime}(z)}{f(z)-b_{i}} d z$ and $\left(e^{*}\right)^{\vee}\left(i d_{\mathbb{C}}\right)=\left[\ldots\left[\frac{d z}{z-b_{i_{1}}} \ldots\left[\ldots, \frac{d z}{z-b_{i_{k}}}\right] \ldots\right]\right.$. Let us define maps

$$
i_{\mathbb{X}}: \operatorname{Lie}\left(A^{1}(\mathbb{X})\right) \rightarrow L\left(\mathbb{C}(z)^{*}\right) \quad \text { and } \quad i_{\mathbb{Y}}: \operatorname{Lie}\left(A^{1}(\mathbb{Y})\right) \rightarrow L\left(\mathbb{C}(z)^{*}\right)
$$

by the formula $i_{\mathbb{X}}\left(\frac{d z}{z-a_{i}}\right)=\left(z-a_{i}\right)$. Observe that the diagram

$$
\begin{array}{cccl}
\operatorname{Lie}\left(A^{1}(\mathbb{X})\right) & \stackrel{\left(f^{*}\right)}{\longleftarrow} & \operatorname{Lie}\left(A^{1}(\mathbb{Y})\right) & \stackrel{\left(e^{*}\right)^{\vee}}{\longleftarrow} \mathbb{C}^{\vee} \\
\downarrow i_{\mathbb{X}} & & \downarrow i_{\mathbb{Y}} & \swarrow \tilde{e}:=i_{\mathbb{Y}} \circ\left(e^{*}\right)^{\vee} \\
L\left(\mathbb{C}(z)^{*}\right) & \stackrel{\left(f^{\sharp}\right)}{\longleftarrow} & L\left(\mathbb{C}(z)^{*}\right) &
\end{array}
$$


commutes, where $f^{\sharp}(z-a)=f(z)-a$ and $\tilde{e}\left(i d_{\mathbb{C}}\right)=\left[\ldots\left[z-b_{i_{1}}, \ldots\left[\ldots, z-b_{i_{k}}\right] \ldots\right]\right.$. The maps $i_{\mathbb{X}}$ and $i_{\mathbb{Y}}$ are inclusions, hence the condition ii') is equivalent to the condition $\sum_{i=1}^{N} n_{i} \cdot\left(f_{i}\right)^{\sharp} \circ \tilde{e}_{i}=0$. This last condition is equivalent to the condition $\sum_{i=1}^{N} n_{i}$. $\left(f_{i}\right)^{\sharp}\left(\tilde{e}_{i}\left(i d_{\mathbb{C}}\right)\right)=0$. Observe that $\left(f_{i}\right)^{\sharp}\left(\tilde{e}_{i}\left(i d_{\mathbb{C}}\right)\right)=b_{\mathbb{Y}}\left(e_{i}\right)\left[f_{i}\right]$. Hence we get that ii) and iii) are equivalent.

\section{$\S 14$. Subgroups of $\operatorname{Aut}\left(\pi_{2}(\mathbb{X})\right)$.}

Let us set $L^{\prime}:=L(\mathbb{X}), L^{\prime \prime}:=\left[L^{\prime}, L^{\prime}\right]$ and $L_{2}(\mathbb{X}):=L(\mathbb{X}) / L^{\prime \prime}$. Then the Lie algebra $L_{2}(\mathbb{X})$ equipped with the multiplication given by the Baker-Campbell-Hausdorff formula is the group $\pi_{2}(\mathbb{X})$ from section 10 .

Let $p: \pi_{2}(\mathbb{X}) \rightarrow \pi_{2}(\mathbb{X}) / \Gamma^{3} \pi_{2}(\mathbb{X})$ be the natural projection. The map $p$ induces $p_{*}: \operatorname{Aut}\left(\pi_{2}(\mathbb{X})\right) \rightarrow \operatorname{Aut}\left(\pi_{2}(\mathbb{X}) / \Gamma^{3} \pi_{2}(\mathbb{X})\right)$. Let $\operatorname{Aut}^{*}\left(\pi_{2}(\mathbb{X})\right):=\left\{f \in \operatorname{Aut}\left(\pi_{2}(\mathbb{X})\right) \mid \exists \alpha \in\right.$ $\left.\mathbb{C}^{*}, f(X)=\alpha X, f(Y) \approx \alpha Y, f(Z) \sim \alpha Z(Z=-X-Y)\right\}(\approx$ is a conjugation by an elemt of $\left[\pi_{2}(\mathbb{X}), \pi_{2}(\mathbb{X})\right]$ and $\sim$ is a conjugation by an element of $\pi_{2}(\mathbb{X})$ ). Let $\mathcal{C}: G_{m} \rightarrow$ $\operatorname{Aut}\left(\pi_{2}(\mathbb{X}) / \Gamma^{3} \pi_{2}(\mathbb{X})\right)$ be given by $\mathcal{C}_{t}(X)=t X, \mathcal{C}_{t}(Y)=t Y$. We shall investigate liftings of $\mathcal{C}$ to $\operatorname{Aut}^{*}\left(\pi_{2}(\mathbb{X})\right)$. We recall that elements $X, Y$ and $\left.\left.\left.Y X^{i} Y^{j-1}:=(\ldots(Y, X) X) \ldots X\right) Y\right) \ldots Y\right)$ $i=1, \ldots, j=1, \ldots$ form a topological base of $L_{2}(\mathbb{X})$.

Notation. If $\exp \left(\sum f_{i, j} X^{i} Y^{j}\right)=1+\sum_{n=1, m=1} F_{n m} X^{n} Y^{m}$, then $(\omega, \exp (\ldots)):=\omega+$ $\left.\left.\sum_{n=1, m=1} F_{n, m}(\ldots(\omega, X) \ldots X) Y\right) \ldots Y\right)$.

We shall investigate pro-algebraic subgroups of $\operatorname{Aut}^{*}\left(\pi_{2}(\mathbb{X})\right)$ and $\left.\operatorname{Aut}^{*}\left(\pi^{2}\right)\right)$ and algebraic subgroups of $\mathrm{Aut}^{*}\left(\pi^{2} / \Gamma^{n+2} \pi^{2}\right)$.

\section{Proposition 14.1.}

i) Let $\Phi: G_{m} \rightarrow \operatorname{Aut}^{*}\left(\pi_{2}(\mathbb{X})\right)$ be given by

$$
\begin{aligned}
& \Phi_{t}(X)=t X \\
& \Phi_{t}(Y)=\left(t Y, \exp \left(\sum_{n=2}^{\infty} \sum_{\substack{i+j=n \\
i \geq 1, j \geq 1}} c_{i, j}\left(1-t^{n}\right) X^{i} Y^{j}\right)\right), \quad \text { where } c_{i, j} \in \mathbb{C} .
\end{aligned}
$$

Then $\Phi$ is a homomorphism. 
ii) All homomorphisms $\Phi$ of $G_{m}$ into Aut* ${ }^{*}\left(\pi_{2}(\mathbb{X})\right)$ such that $p_{*} \circ \Phi=\mathcal{C}$ are of this form.

iii) All one-dimensional subgroups $G$ of $\operatorname{Aut}^{*}\left(\pi_{2}(\mathbb{X})\right)$, which the map $p_{*}$ projects onto $\mathcal{C}\left(G_{m}\right)$ are of this form.

iv) The group $G$ is defined over a subfield $k$ of $\mathbb{C}$ if and only if all $c_{i j}$ are in $k$.

Proof. The point i) is a straightforward verification. To show point ii) we can assume that $\Phi$ has a form $\Phi_{t}(Y)=\left(t Y, \exp \left(\sum_{i \geq 1, j \geq 1} f_{i, j}(t) X^{i} Y^{j}\right)\right)$, where $f_{i, j}(t) \in \mathbb{C}\left[t, t^{-1}\right]$ are Laurent polynomials. Assume that $\Phi$ has a form $\Phi_{t}(Y)=\left(t Y, \exp \left(\sum_{n=2}^{N-1} \sum_{i+j=n} c_{i, j}\left(1-t^{n}\right) X^{i} Y^{j}+\right.\right.$ $\left.\left.\sum_{i+j=N} f_{i, j}(t) X^{i} Y^{j}+\sum_{i+j>N} F_{i, j}(t) X^{i} Y^{j}\right)\right)$. Then one gets $f_{i, j}(t)+t^{N} f_{i, j}(s)=f_{i, j}(s \cdot t)$. This implies $f_{i, j}(t)=c_{i, j}\left(1-t^{N}\right)$. Hence the point ii) is proved. If a subgroup of $\operatorname{Aut}^{*}\left(\pi_{2}(\mathbb{X})\right)$ is one dimensional then the coefficients $f_{i, j}$ are algebraic functions of $t$. They cannot be multivalued functions because then the dimension of the subgroup would be greater than 1. Hence $f_{i, j}$ are Laurent polynomials of $t$. Now the point iii) follows from the proof of ii). The last point is obvious because char $k=0$.

In section 6 we need also results about the subgroups of $\operatorname{Aut}\left(\pi^{2} / \Gamma^{n+1} \pi^{2}\right)$ and $\operatorname{Aut}\left(\pi^{2}\right)$. Let $\mathcal{C}: G_{m} \rightarrow \operatorname{Aut}\left(\pi^{2} / \Gamma^{3} \pi^{2}\right)$ be given by $\mathcal{C}_{t}(X)=t X, \mathcal{C}_{t}(Y)=t Y$. Let $\left(p^{n}\right)_{*}: \operatorname{Aut}\left(\pi^{2} / \Gamma^{n+2} \pi^{2}\right) \rightarrow$ $\operatorname{Aut}\left(\pi^{2} / \Gamma^{3} \pi^{2}\right)$ and $p_{*}: \operatorname{Aut}\left(\pi^{2}\right) \rightarrow \operatorname{Aut}\left(\pi^{2} / \Gamma^{3} \pi^{2}\right)$ be induced by projections of $\pi^{2} / \Gamma^{n+2} \pi^{2}$ and $\pi^{2}$ onto $\pi^{2} / \Gamma^{3} \pi^{2}$.

Corollary 14.2. All one dimensional subgroups $G$ of $\operatorname{Aut}\left(\pi^{2} / \Gamma^{n+2} \pi^{2}\right) \quad\left(\operatorname{resp} . \operatorname{Aut}\left(\pi^{2}\right)\right)$ which the map $\left(p^{n}\right)_{*} \quad\left(\right.$ resp. $\left.\quad p_{*}\right)$ projects onto $\mathcal{C}\left(G_{m}\right)$ are of the form $G=\left\{f_{t} \in\right.$ $\operatorname{Aut}\left(\pi_{2} / \Gamma^{n+2} \pi_{2}\right)\left(\operatorname{resp}\right.$. Aut $\left(\pi^{2}\right)\left|f_{t}(X)=t X, f_{t}(Y)=t Y+\sum_{k=1}^{n(\text { resp. } \infty)} c_{k}\left(t-t^{k+2}\right)\left((Y X) Y^{k}\right)\right| t \in$ $\left.\mathbb{C}^{*}\right\}$. The group $G$ is defined over a subfield $k$ of $\mathbb{C}$ if and only if all $c_{i}$ 's are in $k$.

The subgroup $G$ of $\operatorname{Aut}\left(\pi^{2} / \Gamma^{n+2} \pi^{2}\right)$ considered in the corollary we shall denote by $G\left(c_{1}, c_{2}, \ldots, c_{n}\right)$. The subgroup $G$ of $\operatorname{Aut}\left(\pi^{2}\right)$ considered in the corollary we shall denote by $G\left(\left(c_{i}\right)_{i=1}^{\infty}\right)=G\left(c_{1}, c_{2}, \ldots, c_{n}, \ldots\right)$.

Let $N$ be the set of natural numbers and let $c: N \times N \rightarrow \mathbb{C}$ be a function into complex numbers. For each $n$ let $l_{1}^{n}, \ldots, l_{k_{n}}^{n}$ be linear forms with complex coefficients in variables $x_{i, j}$, where $i+j=n, i \geq 1, j \geq 1$. Let $L:=\left\{\left(l_{1}^{n}, \ldots, l_{k_{n}}^{n}\right)_{n \in N}\right\}$. Let $\mathbb{C}^{n-1}=$ 
$\sum_{\substack{i+j=n \\ i \geq i, j \geq 1}} \mathbb{C}\left(\left(a_{1, n-1}, a_{2, n-2) \ldots}\right) \in \mathbb{C}^{n-1}\right)$ and let $V_{n}$ be a set of common zeroes of $l_{1}^{n}, \ldots, l_{k_{n}}^{n}$. Observe that for each $n$, the sequence $\left(c_{i j}\right)_{i+j \in n} \in \mathbb{C}^{n-1}$.

\section{Proposition 14.3.}

i) Let $G(c, L):=\left\{f \in \operatorname{Aut}^{*}\left(\pi_{2}(\mathbb{X})\right) \mid f(X)=t X, f(Y)=\left(t Y, \exp \left(\sum_{n=2}^{\infty} \sum_{i+j=n}\left(c_{i j}\left(1-t^{n}\right)+\right.\right.\right.\right.$

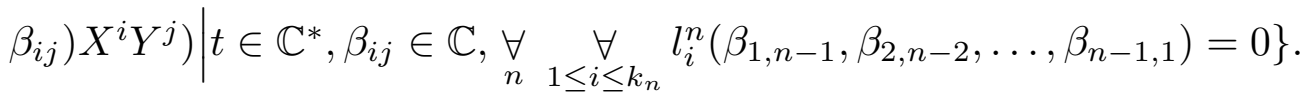

Then $G(c, L)$ is a subgroup of $\operatorname{Aut}^{*}\left(\pi_{2}(\mathbb{X})\right)$.

ii) Any subgroup $G$ of $\operatorname{Aut}^{*}\left(\pi_{2}(\mathbb{X})\right)$, whose projection onto $\operatorname{Aut}^{*}\left(\pi_{2}(\mathbb{X}) / \Gamma^{3} \pi_{2}(\mathbb{X})\right)$ is $\mathcal{C}\left(G_{m}\right)$ is of this form.

iii) Two subgroups $G(c, L)$ and $G\left(c^{\prime}, L^{\prime}\right)$ coincides if and only if for each $n V_{n}=V_{n}^{\prime}$ and $\left(c_{i j}\right)_{i+j=n}+V_{n}=\left(c_{i j}^{\prime}\right)_{i+j=n}+V_{n}$.

iv) The group $G(c, L)$ is defined over the subfield $k$ of $\mathbb{C}$ if and only if for each $n$ the vector space $V_{n}$ and the affine space $\left(c_{i j}\right)_{i+j=n}+V_{n}$ are defined over $k$.

Proof. The point i) is a standard checking. Let $G$ be as in the point i). Let $G_{1}:=$ $\operatorname{ker}\left(p_{*}: G \rightarrow \operatorname{Aut}\left(\pi_{2}(\mathbb{X}) / \Gamma^{3} \pi_{2}(\mathbb{X})\right)\right.$. Any element $f \in G_{1}$ is of the form $f(X)=X, f(Y)=$ $\left(Y, \exp \left(\sum_{n=2}^{\infty} \sum_{i+j=n} f_{i, j} X^{i} Y^{j}\right)\right)$. Observe that $f \rightarrow\left(\left(f_{i, j}\right)_{i+j=n}\right)_{n=2,3, \ldots, N}$ defines a homomorphism $G_{1} \rightarrow \sum_{n=2}^{N} \sum_{i+j=n} G_{a}$. Hence there are only linear relations between various $f_{i j}$ 's. The group $G$ is an extension of $G_{m}$ by the pro-unipotent group $G_{1}$, hence there is a lifting $\phi: G_{m} \rightarrow G$ of $\mathcal{C}$. If we calculate $f_{i, j}$ coefficients of $\phi_{t} \circ f \circ \phi_{t}^{-1}$ we get that it can be linear relations only between $\left(f_{i, n-i}\right)_{i=1, \ldots, n-1}$. Hence for each $n$ we have a finite number of linear forms $l_{1}^{n}, \ldots, l_{k_{n}}^{n}$ in variables $x_{1, n-1}, x_{2, n-2}, \ldots, x_{n-1,1}$ such that

$$
\begin{gathered}
G_{1}=\left\{f \mid f(X)=X, f(Y)=\left(Y, \exp \left(\sum_{n=2}^{\infty} \sum_{i+j=n} \beta_{i, j} X^{i} Y^{j}\right) \mid\right.\right. \\
\left.\beta_{i, j} \in \mathbb{C}, \underset{n}{\forall} \underset{1 \leq i \leq k_{n}}{\forall} l_{i}^{n}\left(\beta_{1, n-1}, \beta_{2, n-2}, \ldots\right)=0\right\} .
\end{gathered}
$$

Let $L=\left\{\left(l_{1}^{n}, \ldots, l_{k_{n}}^{n}\right)_{n \in N}\right\}$. The group $\Phi\left(G_{m}\right)$ is as in Proposition 14.1.i) for some function $c: N \times N \rightarrow \mathbb{C}$. Observe that $G_{1} \circ \Phi\left(G_{m}\right)=G(c, L)$. Hence by dimensional reasons $G=G(c, L)$. The points iii) and iv) are evident. 
Let $\left(\varepsilon_{i}\right)_{i=1}^{\infty}$ be a sequence such that $\varepsilon_{i} \in\{0,1\}$ for each $i$. Let $\left(c_{i}\right)_{i=1}^{\infty}$ be a sequence of complex numbers. We set $G\left(\left(c_{i}\right)_{i=1}^{\infty} \mid\left(\varepsilon_{i}\right)_{i=1}^{\infty}\right)=G\left(c_{1}, c_{2}, \ldots \mid \varepsilon_{1}, \varepsilon_{2}, \ldots\right):=\{f=$ $f_{t,\left(\varepsilon_{i} \beta_{i}\right)_{i=1}^{\infty}} \in \operatorname{Aut}\left(\pi^{2}\right)\left|f(X)=t \cdot X, f(Y)=t \cdot Y+\sum_{i=1}^{\infty}\left(\left(\varepsilon_{i} \beta_{i}\right)+\left(1-\varepsilon_{i}\right) c_{i}\left(t-t^{i+2}\right)\right)\left((Y X) Y^{i}\right)\right| t \in$ $\mathbb{C}^{*}, \beta_{i} \in \mathbb{C}$ for $\left.i=1,2, \ldots\right\}$. If we replace in the above definition $\infty$ by $n$ we get a subgroup, denoted by $G\left(c_{1}, \ldots, c_{n} \mid \varepsilon_{1}, \ldots, \varepsilon_{n}\right)$ of $\operatorname{Aut}\left(\pi^{2} / \Gamma^{n+2} \pi^{2}\right)$. Let $\delta\left(\left(\varepsilon_{i}\right)_{i=1}^{n}\right)$ be a number of $\varepsilon_{i}$ equal to 1 . Then $\operatorname{dim} G\left(c_{1}, \ldots, c_{n} \mid \varepsilon_{1}, \ldots, \varepsilon_{n}\right)=\delta\left(\left(\varepsilon_{i}\right)_{i=1}^{n}\right)+1$.

Corollary 14.4. All subgroups of $\operatorname{Aut}^{*}\left(\pi^{2} / \Gamma^{n+2} \pi^{2}\right)\left(\operatorname{resp}\right.$. Aut $\left.^{*}\left(\pi^{2}\right)\right)$, which the map $\left(p^{n}\right)_{*}$ (resp. $\left.p_{*}\right)$ projects onto $\mathcal{C}\left(G_{m}\right)$ are of the form $G\left(c_{1}, \ldots, c_{n} \mid \varepsilon_{1}, \ldots, \varepsilon_{n}\right)\left(\operatorname{resp} . G\left(\left(c_{i}\right)_{i=1}^{\infty} \mid\left(\varepsilon_{i}\right)_{i=1}^{\infty}\right)\right)$. The group is defined over a subfield $k$ of $\mathbb{C}$ if and only if all numbers $\left(1-\varepsilon_{i}\right) c_{i}$ are in $k$.

The subgroup $\left\{f_{t} \in \operatorname{Aut}\left(\pi^{2} / \Gamma^{n+2} \pi^{2}\left|f_{t}(X)=t X_{1} f_{t}(Y)=t Y\right| t \in \mathbb{C}^{*}\right\}\right.$ of $\operatorname{Aut}\left(\pi^{2} / \Gamma^{n+2} \pi^{2}\right)$ we shall denote by $G_{0}$. Let $\delta: \mathbb{C} \rightarrow\{0,1\}$ be a map defined by $\delta(0)=0, \delta(z)=1$ if $z \neq 0$.

Lemma 14.5. Let $f_{0} \in \operatorname{Aut}\left(\pi^{2} / \Gamma^{n+2} \pi^{2}\right)$ be such that $f_{0}(X)=\alpha_{0} X, f_{0}(Y)=\alpha_{0} Y+$ $\sum_{i=1}^{n-1} \beta_{i}^{0}\left((Y X) Y^{i}\right)$. Let $G \subset \operatorname{Aut}\left(\pi^{2} / \Gamma^{n+2} \pi^{2}\right)$ be the smallest algebraic subgroup of $\operatorname{Aut}\left(\pi^{2} / \Gamma^{n+2} \pi^{2}\right)$ such that $G_{0} \subset G$ and $f_{0} \in G$. Then $G=G\left(0,0, \ldots, 0 \mid \delta\left(\beta_{1}^{0}\right), \ldots, \delta\left(\beta_{n-1}^{0}\right)\right)$.

Proof. Let $\mathcal{C}_{t}(X)=t X$ and $\mathcal{C}_{t}(Y)=t Y$ and let $f_{1}=f_{0} \circ \mathcal{C}_{\alpha_{0}}^{-1}$. Then $f_{1}(X)=X$ and $f_{1}(Y)=Y+\sum_{i=1}^{n} \beta_{i}^{0} \alpha_{0}^{-1}\left((Y X) Y^{i-1}\right)$. Let $G_{1} \subset G$ be a subgroup consisting of $h$ such that $h(X)=X, h(Y)=Y+\sum_{i=1}^{n} \beta_{i}\left((Y X) Y^{i-1}\right)$. Assume that for some $i, \beta_{i}^{0} \neq 0$. Then $G_{1} \neq\{I d\}$ because $f_{1} \in G_{1}$. The subgroup $G_{1}$ is contained in $\left(G_{a}\right)^{n}$, hence it is given by a finite number of linear forms. Let $k=\mathcal{C}_{t} \circ h \circ \mathcal{C}_{t}^{-1}$. Then $k(X)=X, k(Y)=Y+\sum_{i=1}^{n} t^{i} \beta_{i}\left((Y X) Y^{i-1}\right)$. Let $l\left(X_{1}, \ldots, X_{n}\right)$ be one of linear forms defining $G_{1}$. Then $l\left(t_{\beta_{1}}, t^{2} \beta_{2}, \ldots, t^{n} \beta_{n}\right)=0$ implies $l \equiv 0$. Hence the $\beta_{1}, \beta_{2}, \ldots, \beta_{n}$, if they are non-zero, they are linearly independent. Hence $G=G\left(0,0, \ldots, 0 \mid \delta\left(\beta_{1}^{0}\right), \ldots, \delta\left(\beta_{n-1}^{0}\right)\right)$.

Corollary 14.6. Let $G \subset \operatorname{Aut}\left(\pi^{2} / \Gamma^{n+2} \pi^{2}\right)$ be the smallest subgroup such that the subgroup $G_{0}:=\left\{f_{t} \in \operatorname{Aut}\left(\pi^{2} / \Gamma^{n+2} \pi^{2}\right)\left|f_{t}(X)=t X, f_{t}(Y)=t Y\right| t \in \mathbb{C}^{*}\right\}$ is contained in $G$ and the subgroup $G\left(c_{1}, \ldots, c_{n}\right)$ is contained in $G$. Then $G=G\left(0, \ldots, 0 \mid \delta\left(c_{1}\right), \delta\left(c_{2}\right), \ldots, \delta\left(c_{n}\right)\right)$.

Proof. One takes any element $f$ of $G\left(c_{1}, \ldots, c_{n-1}\right)$ such that $f(X)=\alpha X$ and $\alpha \neq 1$. 


\section{$\S$ A.1. Malcev completion}

Let $\pi$ be a nilpotent group. Then there exists a connected, affine, nilpotent group scheme $\pi_{0}$ over Spec $\mathbb{Q}$, together with a homomorphism $r_{\mathbb{Q}}: \pi \rightarrow \pi_{0}(\mathbb{Q})$, functorial with respect to morphisms $\pi \rightarrow \pi^{\prime}$ such that

i) $r_{\mathbb{Q}}: \pi \rightarrow \pi_{0}(\mathbb{Q})$ is a rationalization of $\pi$,

ii) for any field $K$ of characteristic zero, the morhpism $r_{K}: \pi \stackrel{r_{\mathbb{Q}}}{\rightarrow} \pi_{0}(\mathbb{Q}) \hookrightarrow \pi_{0}(K)$ is the Malcev $K$-completion of $\pi$ i.e. for any homomorphism $f: \pi \rightarrow G$ of $\pi$ into a nilpotent $K$-group $G$ there is a unique homomorphism of $K$-group $f^{\prime}: \pi_{0}(K) \rightarrow G$ such that $f=f^{\prime} \circ r_{K}$.

If $\pi$ is any group then we set

$$
\pi_{0}:=\lim _{i}\left(\pi / \Gamma^{i} \pi\right)_{0}
$$

The group $\pi_{0}(K)$ together with the morhpism $r_{K}: \pi \rightarrow \pi_{0}(K)$ has a universal property with respect to maps of $\pi$ into pro-nilpotent $K$-groups.

\section{References}

[Ch] K. T. Chen, Algebra of iterated path integrals and fundamental groups, Transactions of the American Mathematical Society, 156, May (1971), $359-739$.

[D1] P. Deligne, Théorie de Hodge II, Publ. Math. IHES, 40, (1971), 5- 58.

[D2] P. Deligne, Hodge Cycles on Abelian Varieties, Lecture Notes in Math. 900, SpringerVerlag 1982, 9 - 100.

[D3] P. Deligne, Equations Différentielles à Points Singuliers Réguliers, Lecture Notes in Math. 163, Springer Verlag 1970.

[D4] P. Deligne, Le Groupe Fondamental de la Droite Projective Moins Trois Points, in Galois Groups over $\mathbb{Q}$, Math. Sc. Res. Ins. Publ. 16, 1989, $79-297$.

[Dr] W. G. Drinfeld, On quasitriangular quasi-Hopf algebras and a group closely connected with $\operatorname{Gal}(\overline{\mathbb{Q}} / \mathbb{Q})$, Leningrad Math. J. 2 (4), (1991), 829 -860.

[KO] N. Katz and T. Oda, On the differentiation of De Rham cohomology classes with respect to parameters, J. Math. Kyoto Univ. 8, (1968), 199 - 213.

[I1] Y. Thara, Automorphisms of pure sphere braid groups and Galois representations, in The Grothendieck Festschrift, Volume II, Birkhäuser, 353 - 373. 
[I2] Y. Ihara, Braids, Galois groups, and some arithmetic functions, Proc. of the ICM 90(I), (1991), $99-120$.

[I3] Y. Ihara, Profinite braid groups, Galois representations and complex multiplications, Annals of Math. 123 (1986), 43 - 106.

[MKS] W. Magnus, A. Karrass, and D. Solitar, Combinatorial Group Theory, Pure and Applied Mathematics, XIII, Interscience Publ. 1966.

[R] R. Ree, Lie Elements and an Algebra Associated with Shuffles, Annals of Mathematics, 68(2), (1958), $210-220$.

[S] T. A. Springer, Galois Cohomology of Linear Algebraic Groups, in Algebraic Groups and Discontinous Subgroups, Proc. of Symp. in Pure Math. IX, AMS, (1966), 149 158.

[W1] Z. Wojtkowiak, Cosimplicial objects in algebraic geometry, in Algebraic $K$-theory and Algebraic Topology, Kluver Academic Publishers, 1993, pp. 287 - 327.

[W2] Z. Wojtkowiak, Monodromy of Polylogarithms and Cosimplicial Spaces, preprint I.H.E.S., 1991.

[W3] Z. Wojtkowiak, A note on the monodromy representation of the canonical unipotent connection on $P^{1}(\mathbb{C}) \backslash\left\{a_{1}, \cdots, a_{n}\right\}$, preprint MPI, Bonn, 1990.

[W4] Z. Wojtkowiak, Functional Equations of Iterated integrals with Regular Singularities, RIMS-967 preprint.

Université de Nice-Sophia Antipolis

Department de Mathématiques

Laboratoire Jean Alexandre Dieudonné

U.R.A. au C.N.R.S., No 168

Parc Valrose - B.P.N ${ }^{\circ} 71$

06108 Nice Cedex 2, France
Research Institute

for Mathematical Sciences

Kyoto University

Kitashirakawa, Sakyo-ku,

Kyoto 606, Japan 